Supporting Information

\title{
Iodine-Catalyzed Cross Dehydrogenative Coupling Reaction: A Regioselective Sulfenylation of Imidazoheterocycles using DMSO as an Oxidant
}

\author{
Yogesh Siddaraju and Kandikere Ramaiah Prabhu* \\ Department of Organic Chemistry, Indian Institute of Science, Bangalore 560 012, Karnataka, India \\ *e-mail: prabhu@orgchem.iisc.ernet.in
}

Fax: (+)91-80-23600529

\section{Contents}

\begin{tabular}{|r|l|r|}
\hline 1. & General Experimental & SI 2 \\
\hline 2. & Optimization studies for heterocyclic thiol and thiones & SI 3 -SI 4 \\
\hline 3. & NMR studies & SI 5 - SI 7 \\
\hline 4. & ${ }^{1} \mathrm{H}$ and ${ }^{13}$ C NMR Spectra & SI 08- SI 75 \\
\hline
\end{tabular}




\section{General experimental}

NMR spectra were recorded on a $400 \mathrm{MHz}$ spectrometer in $\mathrm{CDCl}_{3}$ or DMSO-d6. Tetramethylsilane (TMS; $\delta=0.00 \mathrm{ppm}$ ) for $1 \mathrm{H} \mathrm{NMR}$ in $\mathrm{CDCl}_{3}$, and residual non-deuterated solvent peak $(\delta=2.50 \mathrm{ppm})$ in DMSO-d6, served as an internal standard. The solvent signal $\left(\mathrm{CDCl}_{3}, \delta=77.00 \mathrm{ppm}\right.$; and DMSO-d6, $\left.\delta=39.5 \mathrm{ppm}\right)$ was used as internal standard for ${ }^{13} \mathrm{C}$ NMR. IR spectra were measured using an FT-IR spectrometer. Mass spectra were obtained with a Q-TOF Mass Spectrometer (HRMS). Flash column chromatography was carried out by packing glass columns with commercial silica gel 230-400 mesh (commercial suppliers) and thin-layer chromatography was carried out using silica gel GF-254. All catalysts, reagents and reactants were procured from commercial suppliers. Dichloroethane solvent was distilled over calcium hydride and stored over molecular sieves and used for all procedures. Other solvents, used for work up and chromatographic procedures were purchased from commercial suppliers 


\section{Optimization for thiol-1}

\section{Optimization of solvent}

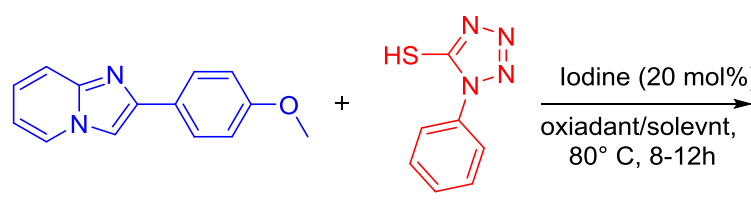

$1 \mathrm{a}(0.5 \mathrm{mmol})$

2a $(0.55 \mathrm{mmol})$

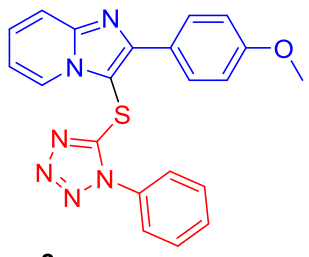

$3 a$

\begin{tabular}{|c|c|c|c|}
\hline entry & oxidant/solvent & time & Isolated yield \\
\hline $\mathbf{1}$ & DMSO & $\mathbf{8 h}$ & $\mathbf{9 0 \%}$ \\
\hline 2 & DMSO (3 equiv), DCE & $12 \mathrm{~h}$ & $82 \%$ \\
\hline 3 & DMSO (3 equiv), EtoAc & $12 \mathrm{~h}$ & trace \\
& & & 61\% \\
\hline 4 & DMSO (3 equiv), ACN & $12 \mathrm{~h}$ & trace \\
\hline 5 & DMSO (3 equiv), Toluene & $12 \mathrm{~h}$ & $\begin{array}{c}\text { trace } \\
\text { (Precipitation of reaction mixture) }\end{array}$ \\
\hline 6 & DMSO (3 equiv), dioxane & $12 \mathrm{~h}$ & ND \\
\hline 7 & DMSO (3 equiv), Trifluoroethanol & $12 \mathrm{~h}$ & ND \\
\hline 8 & DMSO (3 equiv), Water & $12 \mathrm{~h}$ & trace \\
\hline 9 & & & (Precipitation mixture) \\
\hline 10 & DMSO (3 equiv), Ethanol & $12 \mathrm{~h}$ & 22\% \\
\hline 11 & DMSO (3 equiv), DMF & $12 \mathrm{~h}$ & trace \\
\hline 12 & DMSO (3 equiv), NMP & $12 \mathrm{~h}$ & $13 \%$ \\
\hline
\end{tabular}

$\mathrm{ND}=$ Not determined

Reaction with solvents EtoAc, Toluene, dioxane and water starting materials are insoluble and remain heterogeneous

\section{Optimization of catalyst or additive}

\begin{tabular}{|c|c|c|c|c|}
\hline entry & oxidant/solvent & Catalyst/ additive & time & Isolated yield \\
\hline 1 & DMSO & $\mathbf{2 0} \mathbf{~ m o l} \% \mathbf{~ a q . ~ H I ~} \mathbf{5 5 \%})$ & $\mathbf{1 2 h}$ & $\mathbf{7 8 \%}$ \\
\hline 2 & DMSO & 20 mol \% aq, HBr & $12 \mathrm{~h}$ & trace \\
\hline 3 & DMSO & $20 \mathrm{~mol} \%$ NIS & $12 \mathrm{~h}$ & $52 \%$ \\
\hline 4 & DMSO & $20 \mathrm{~mol} \% \mathrm{NBS}$ & $12 \mathrm{~h}$ & trace \\
\hline 5 & DMSO & $20 \mathrm{~mol} \% \mathrm{NCS}$ & $12 \mathrm{~h}$ & trace \\
\hline 6 & DMSO & $20 \mathrm{~mol} \% \mathrm{KI}$ & $12 \mathrm{~h}$ & trace \\
\hline 7 & DMSO & - & $12 \mathrm{~h}$ & NR \\
\hline
\end{tabular}

$\mathrm{NCS}=N$-Chlorosuccinimide

NIS $=N$-Iodosuccinimide

NBS $=N$-Bromosuccinimide

$\mathrm{KI}=$ Potassium iodide 


\section{Optimization of equiv of $1 \mathrm{a}$ and $2 \mathrm{a}$ and mol $\%$ of iodine}

\begin{tabular}{|c|c|c|c|c|c|}
\hline entry & 1a (equiv) & 2a (equiv) & Iodine $(\mathrm{mol} \%)$ & time & Isolated yield \\
\hline 1 & 1 (equiv) & 1.5 (equiv) & $20(\mathrm{~mol} \%)$ & $8 \mathrm{~h}$ & $90 \%$ \\
\hline 2 & 1.5 (equiv) & 1.1 (equiv) & $20(\mathrm{~mol} \%)$ & $8 \mathrm{~h}$ & $86 \%$ \\
\hline 3 & 1 (equiv) & 1.1 (equiv) & $10(\mathrm{~mol} \%)$ & $16 \mathrm{~h}$ & $82 \%$ \\
\hline 4 & 1 (equiv) & 1.1 (equiv) & $30(\mathrm{~mol} \%)$ & $4 \mathrm{~h}$ & $90 \%$ \\
\hline
\end{tabular}

\section{Optimization under Argon and Oxygen atmosphere}

\begin{tabular}{|c|c|c|c|c|c|}
\hline entry & $\begin{array}{c}\text { 1a } \\
\text { (equiv) }\end{array}$ & 2a (equiv) & $\begin{array}{c}\text { Iodine } \\
(\mathrm{mol} \%)\end{array}$ & time & $\begin{array}{c}\text { Isolated } \\
\text { yield }\end{array}$ \\
\hline 1 & 1 (equiv) & 1.1 (equiv) & $20(\mathrm{~mol} \%)$ & Reaction under $\mathrm{O}_{2}$ atmosphere, $8 \mathrm{~h}$ & $91 \%{ }^{a}$ \\
\hline 2 & 1 (equiv) & 1.1 (equiv) & $20(\mathrm{~mol} \%)$ & Reaction under Argon atmosphere, $8 \mathrm{~h}$ & $90 \%^{b}$ \\
\hline
\end{tabular}

${ }^{\mathrm{a}}$ The reaction performed using oxygen balloon

${ }^{b}$ The reaction performed using argon balloon

\section{Optimization for thiones-2}
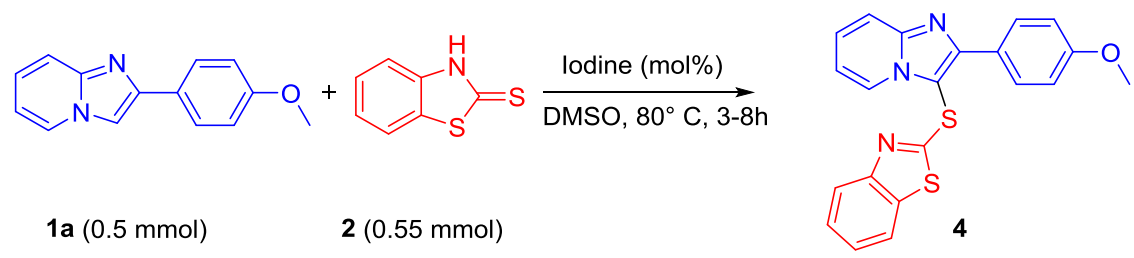

\begin{tabular}{|c|c|c|c|c|c|}
\hline entry & $\mathbf{1 a}$ (equiv) & $\mathbf{2}$ (equiv) & Iodine $(\mathrm{mol} \%)$ & time & Isolated yield \\
\hline 1 & 1 (equiv) & 1.1 (equiv) & $20(\mathrm{~mol} \%)$ & $8 \mathrm{~h}$ & $41 \%$ \\
\hline 2 & 1 (equiv) & 1.1 (equiv) & $50(\mathrm{~mol} \%)$ & $5 \mathrm{~h}$ & $73 \%$ \\
\hline $\mathbf{3}$ & $\mathbf{1}$ (equiv) & $\mathbf{1 . 1}$ (equiv) & $\mathbf{1 0 0}(\mathbf{m o l} \%)$ & $\mathbf{3 h}$ & $\mathbf{9 0 \%}$ \\
\hline
\end{tabular}


NMR STUDIES

EXP-1

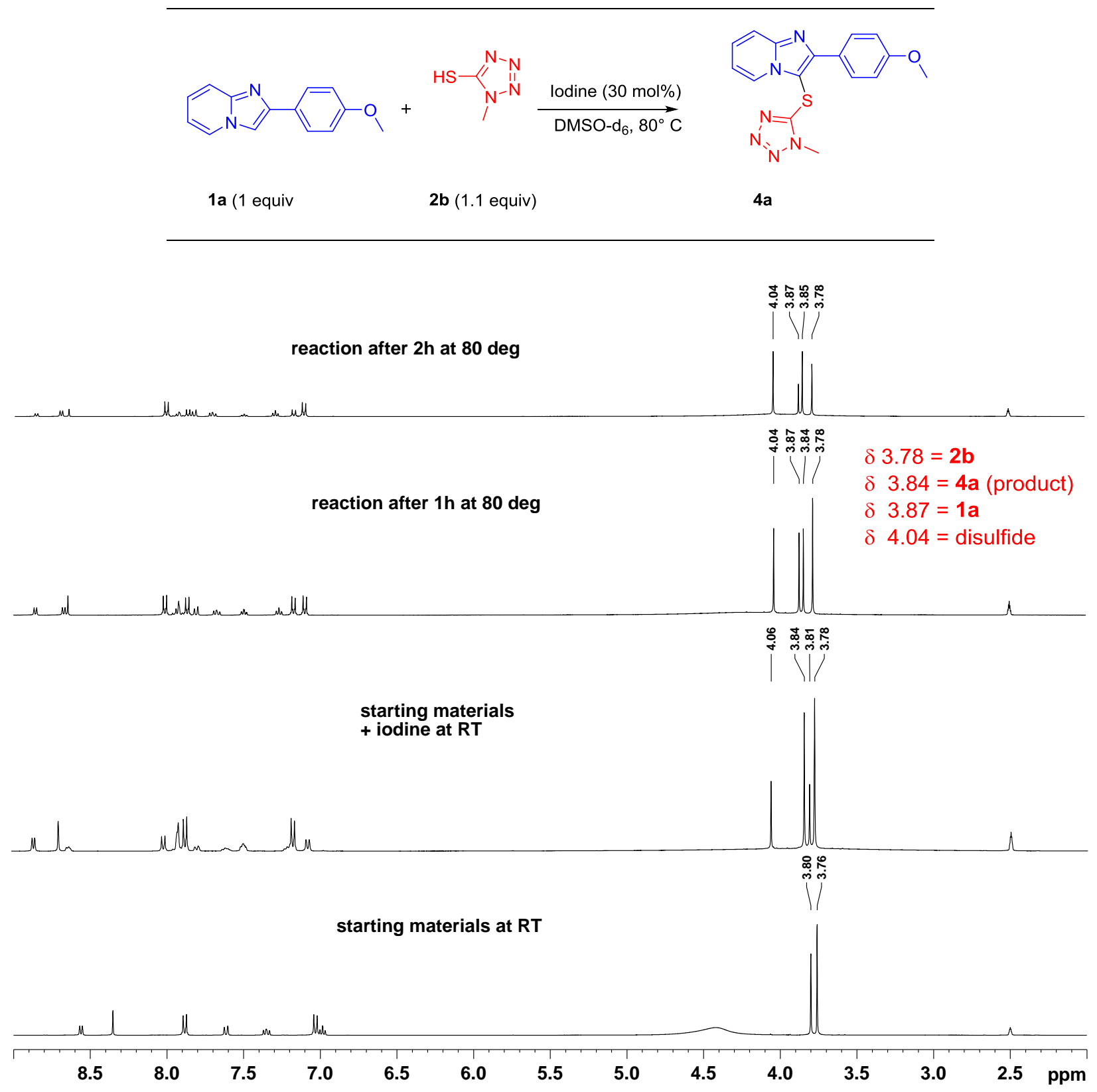


NMR STUDIES

EXP-2

$\delta 6.52$ aromatic peak completely disappears, indicates complete decomposition of 5-methylthiazole-2(3H)-thione

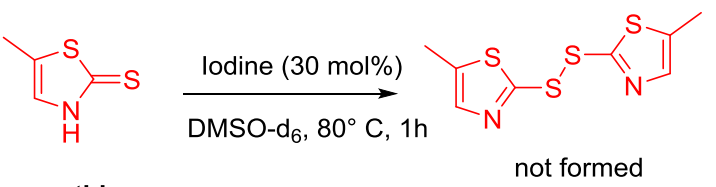

thione complete decomposition of thione

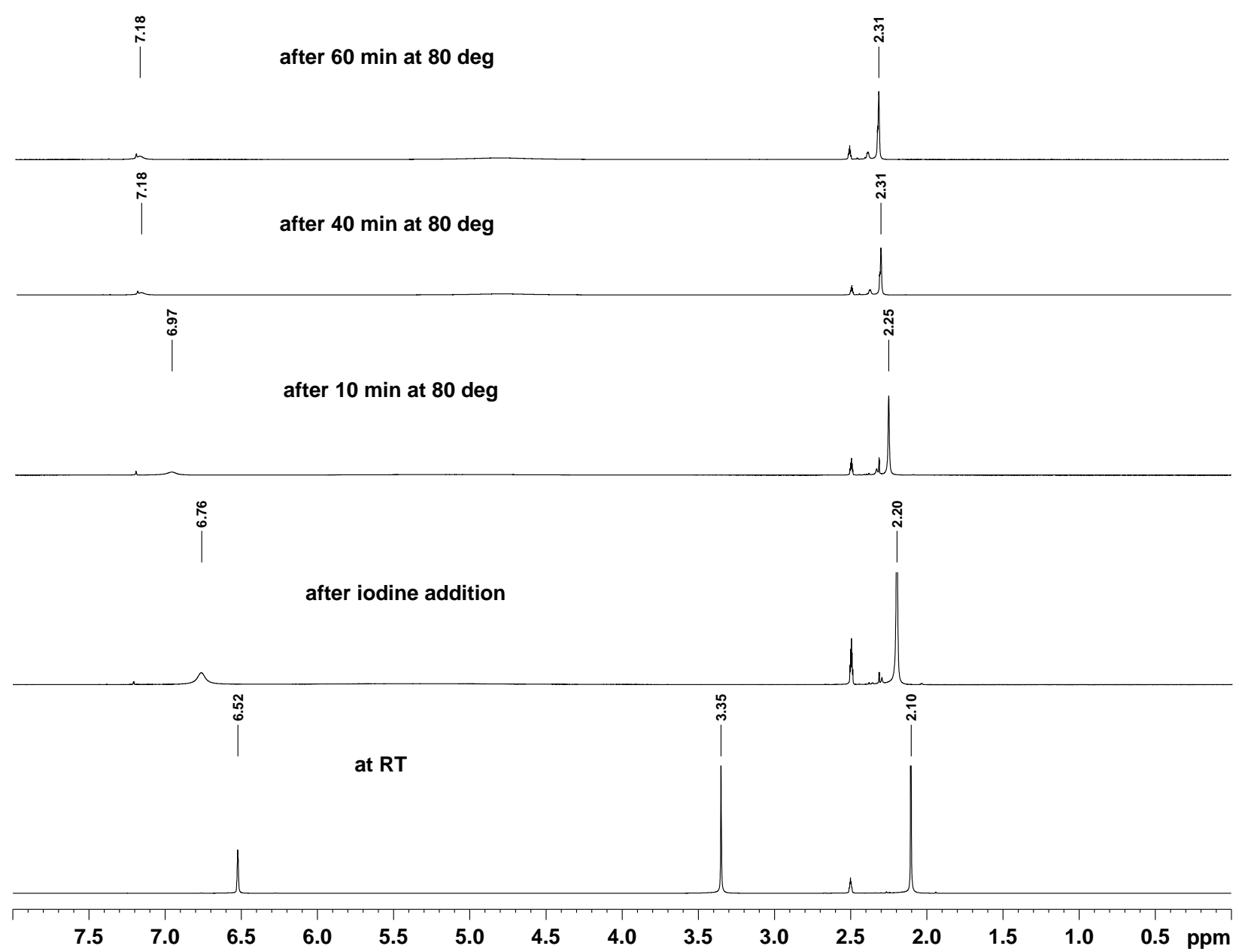


NMR STUDIES

EXP-3

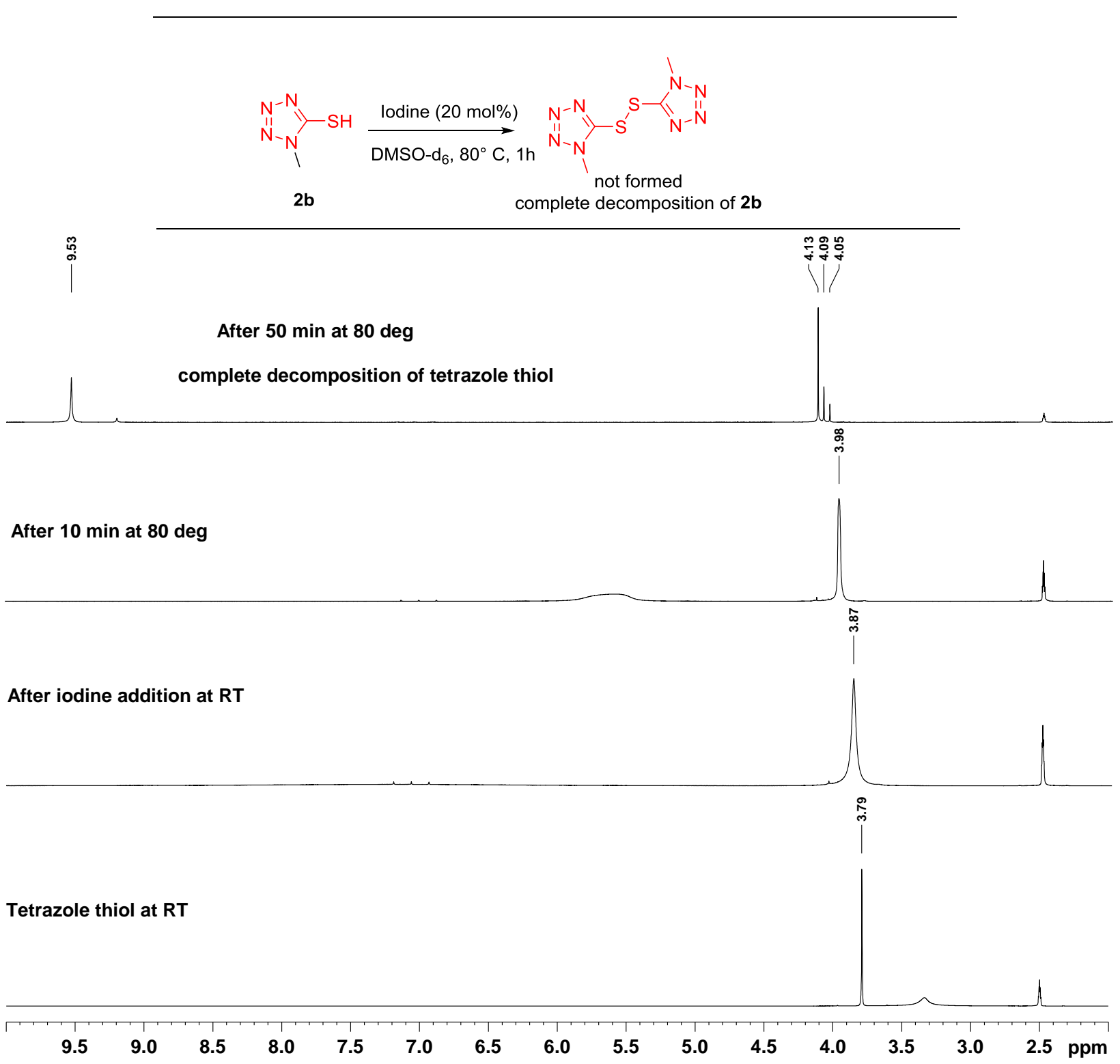




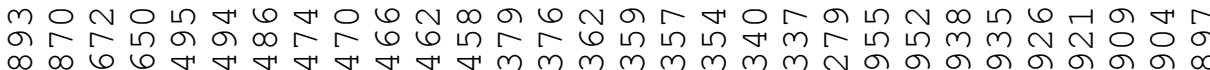

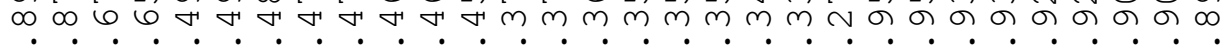

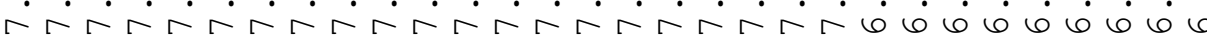
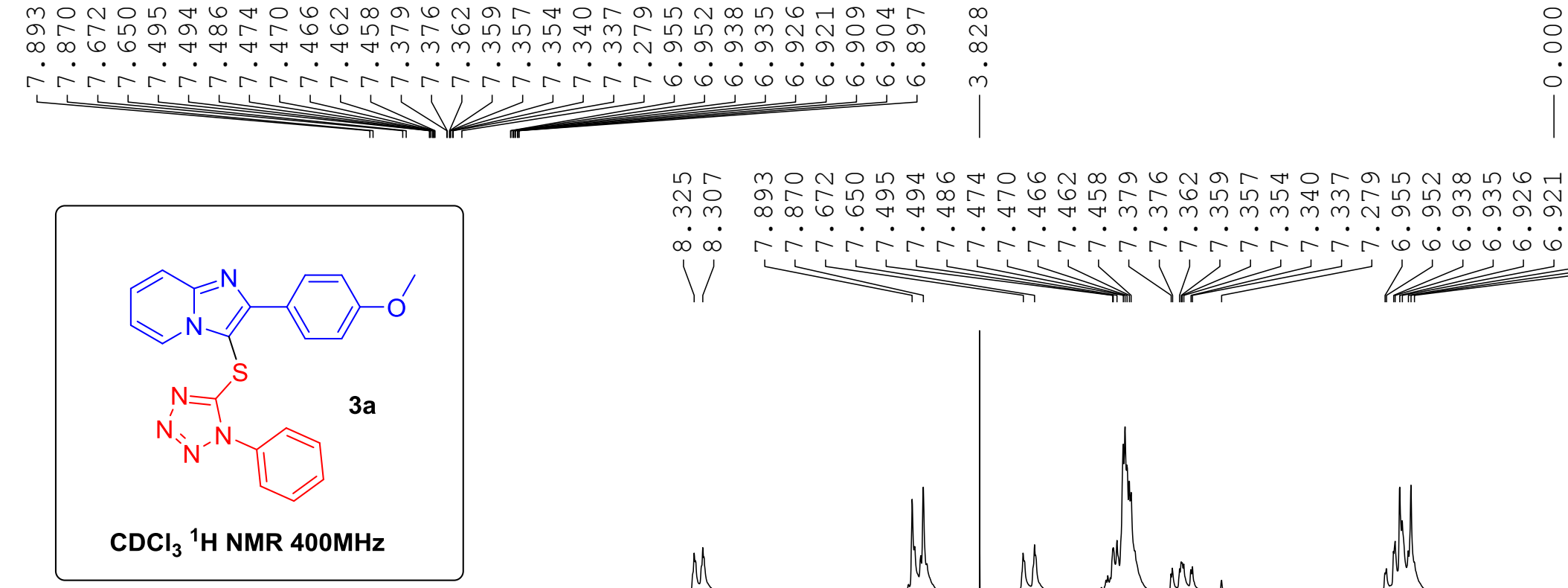

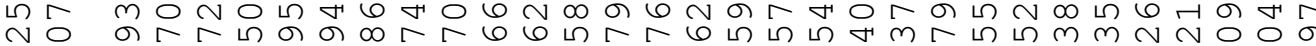

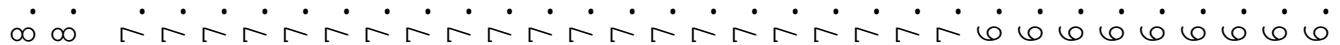
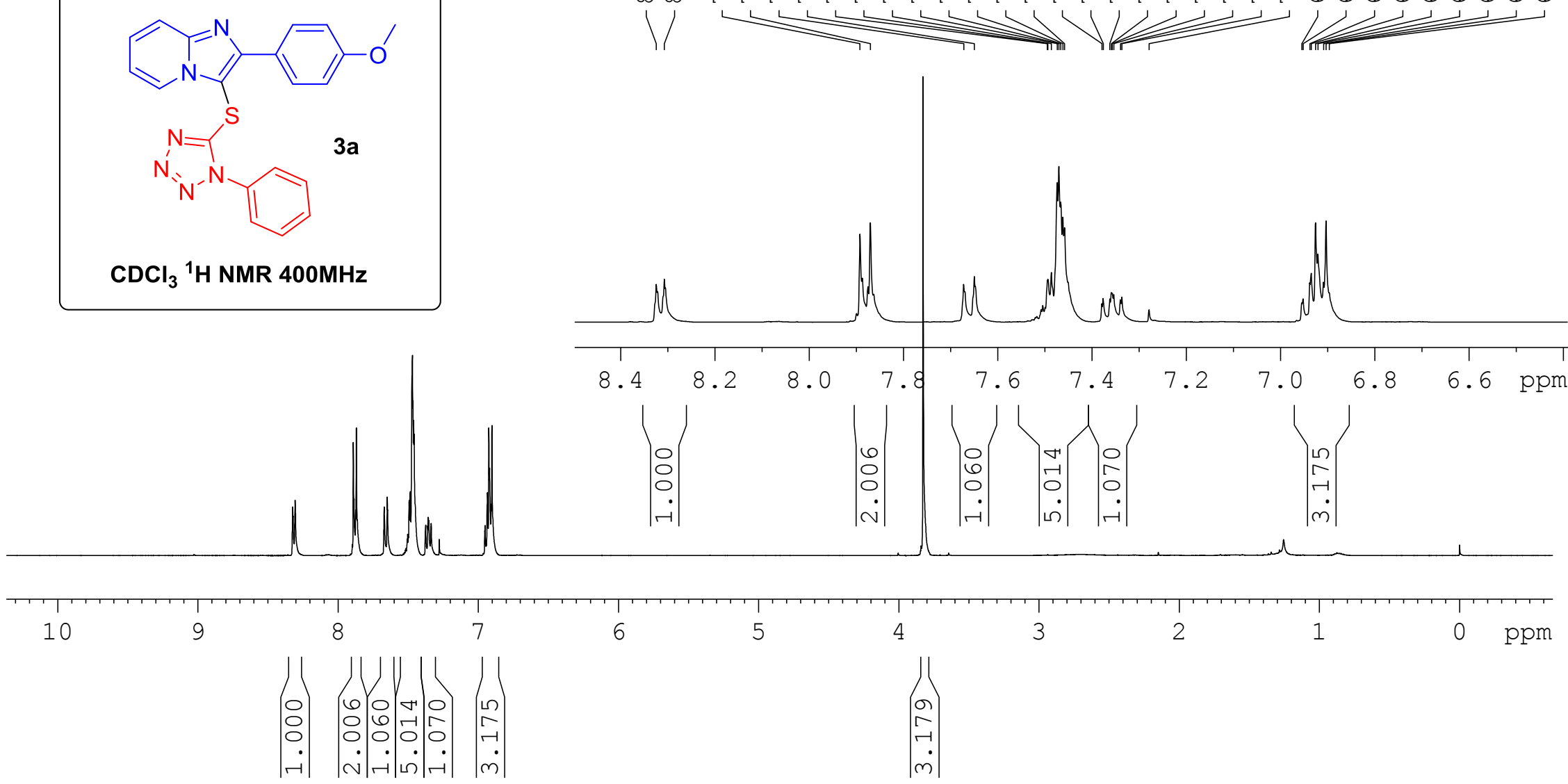


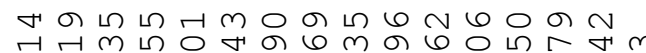

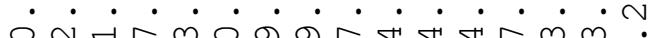

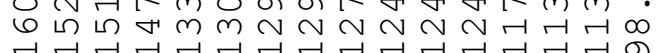
111

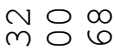

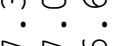
둥
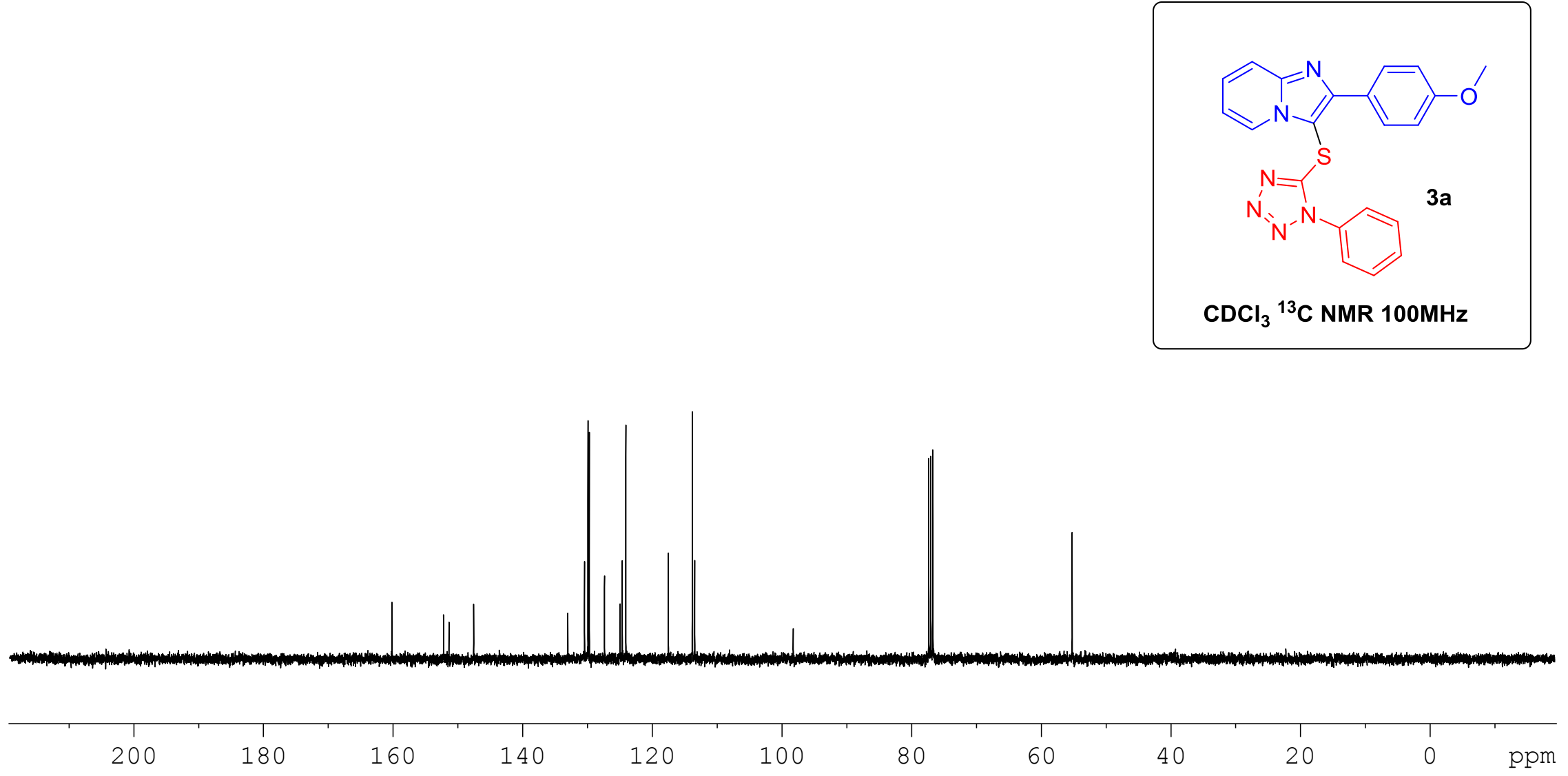
m

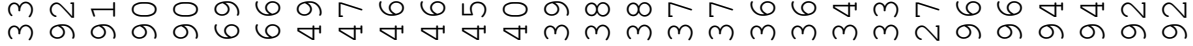

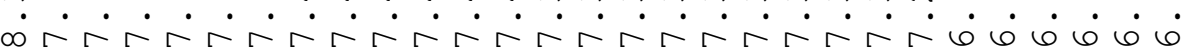
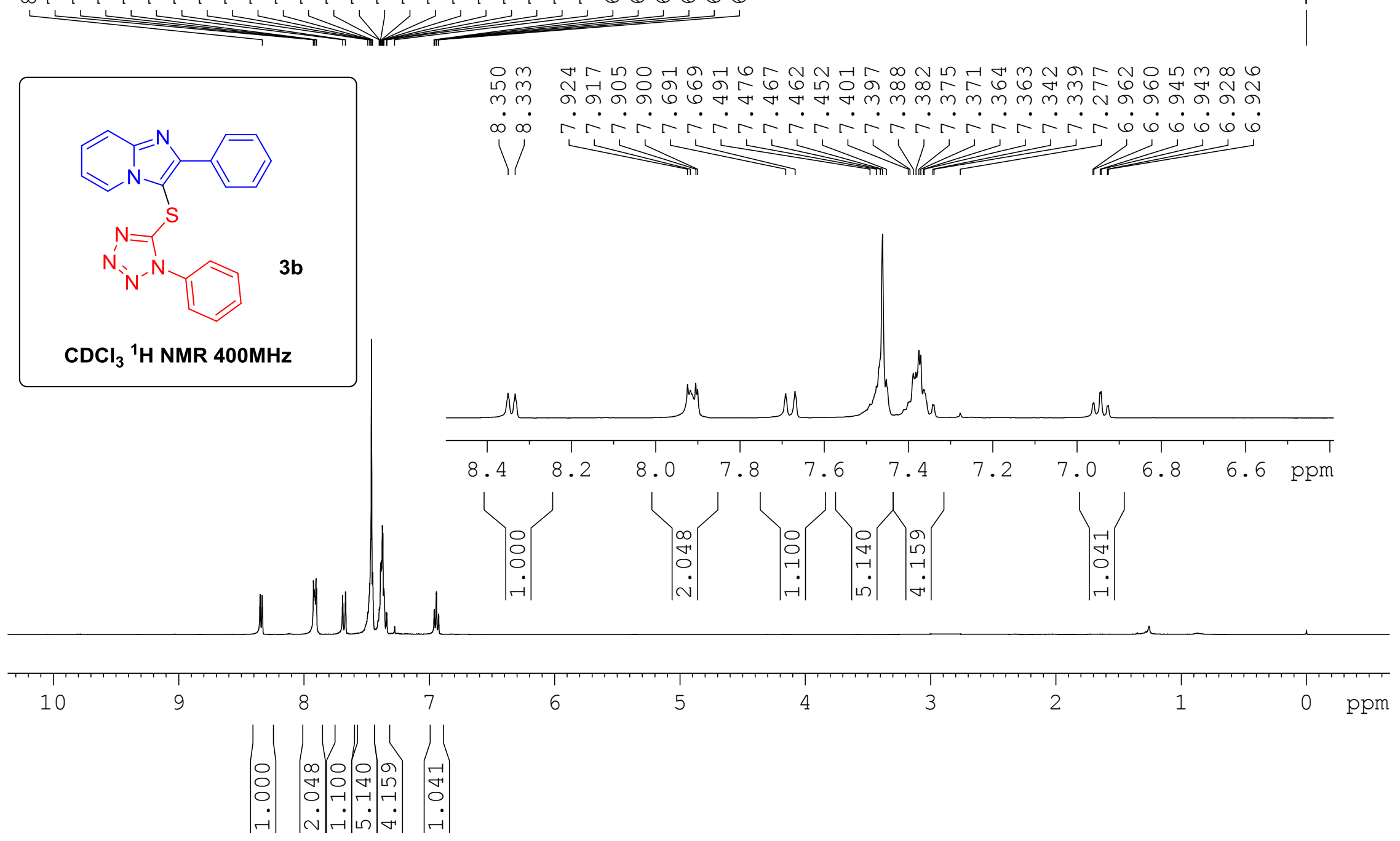


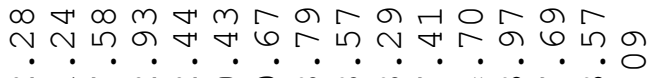

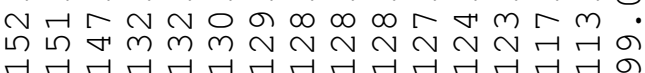

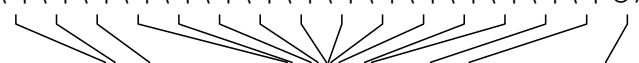

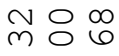

$\therefore \therefore 6$

I

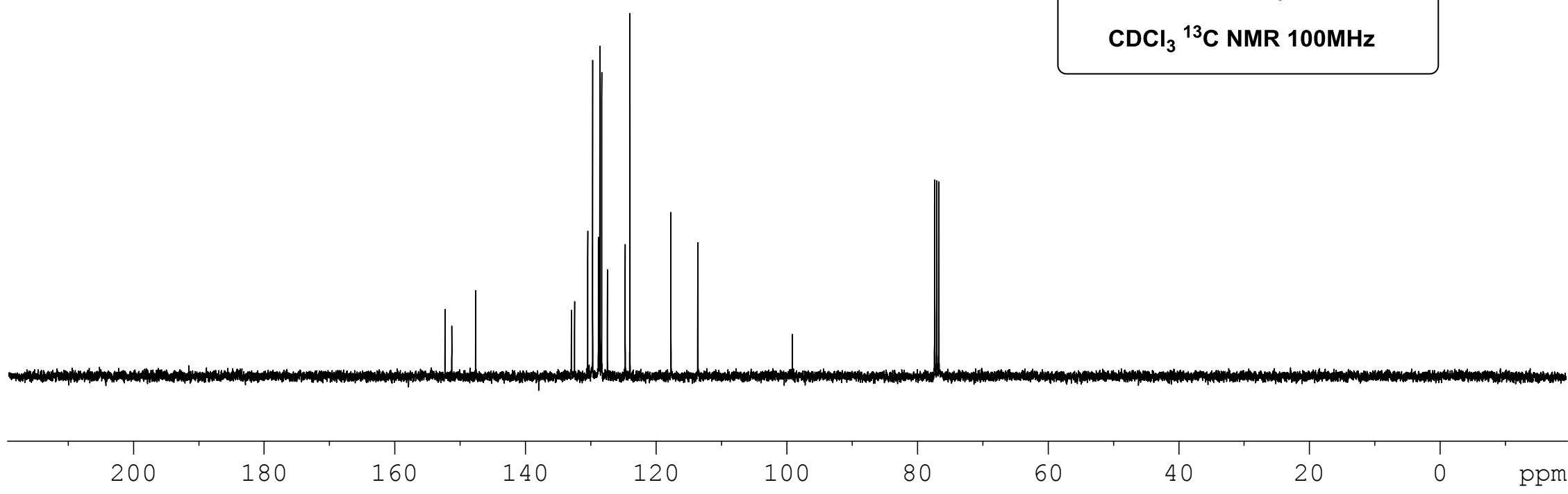


N $N \infty 6$ の

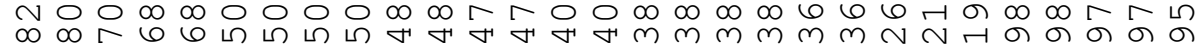

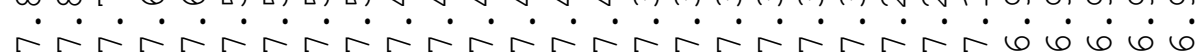

மூm

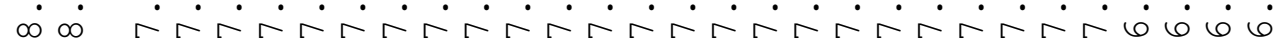

$1 / 2$
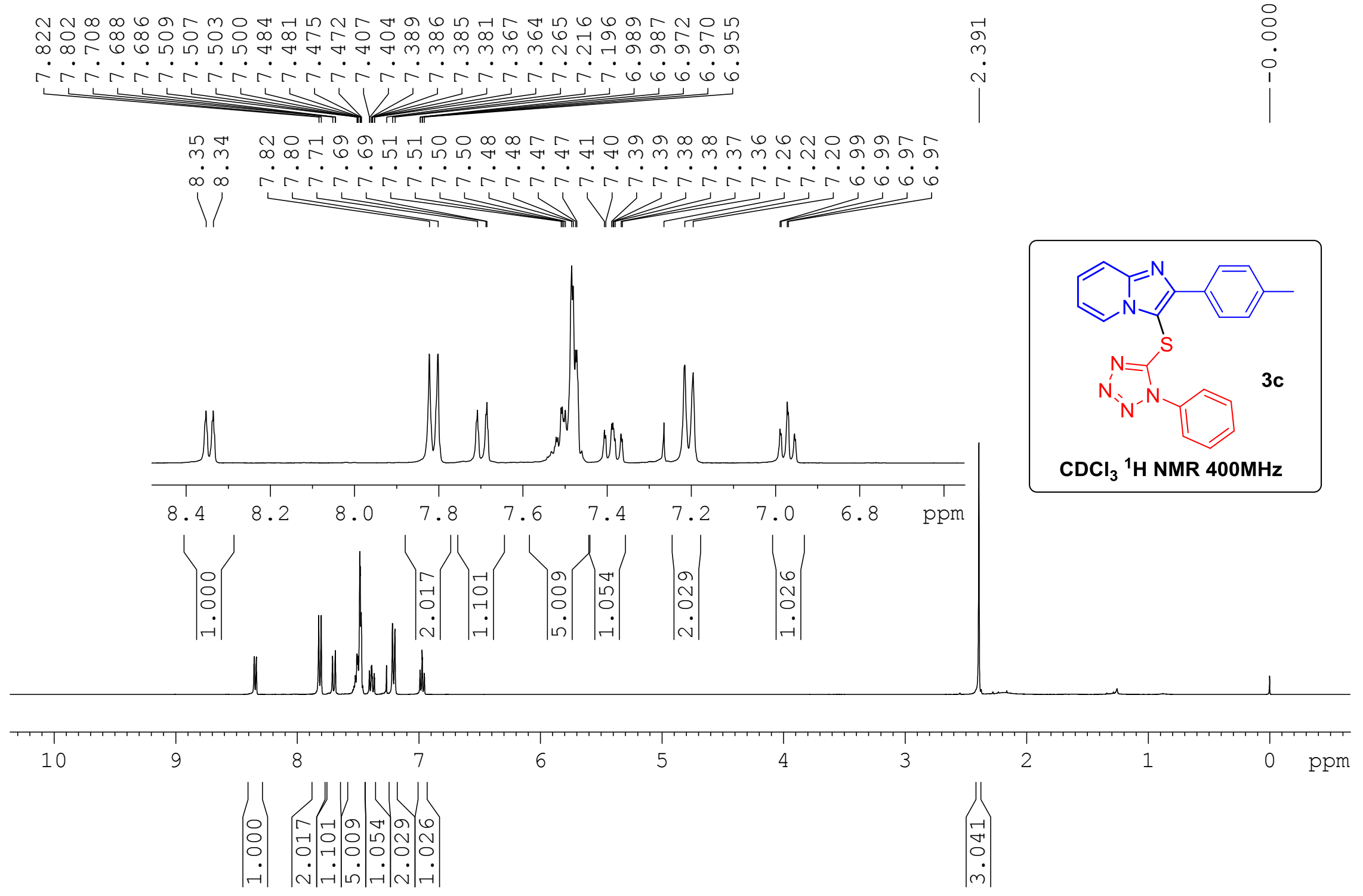


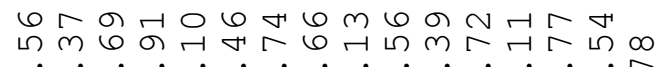

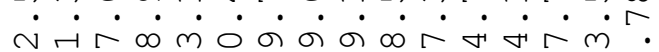
岁

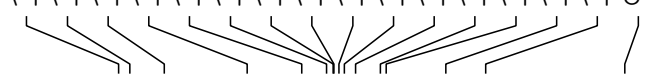

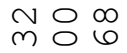

$\therefore \therefore$

$\stackrel{\circ}{m}$

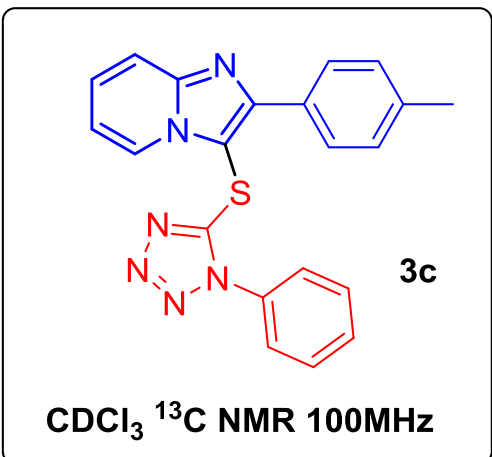

$\mathrm{CDCl}_{3}{ }^{13} \mathrm{C}$ NMR $100 \mathrm{MHz}$

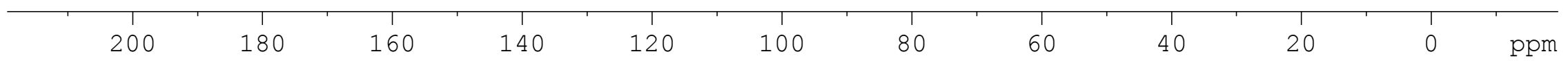


ம

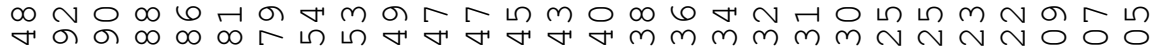

$0 r$
$0.6 r$
0

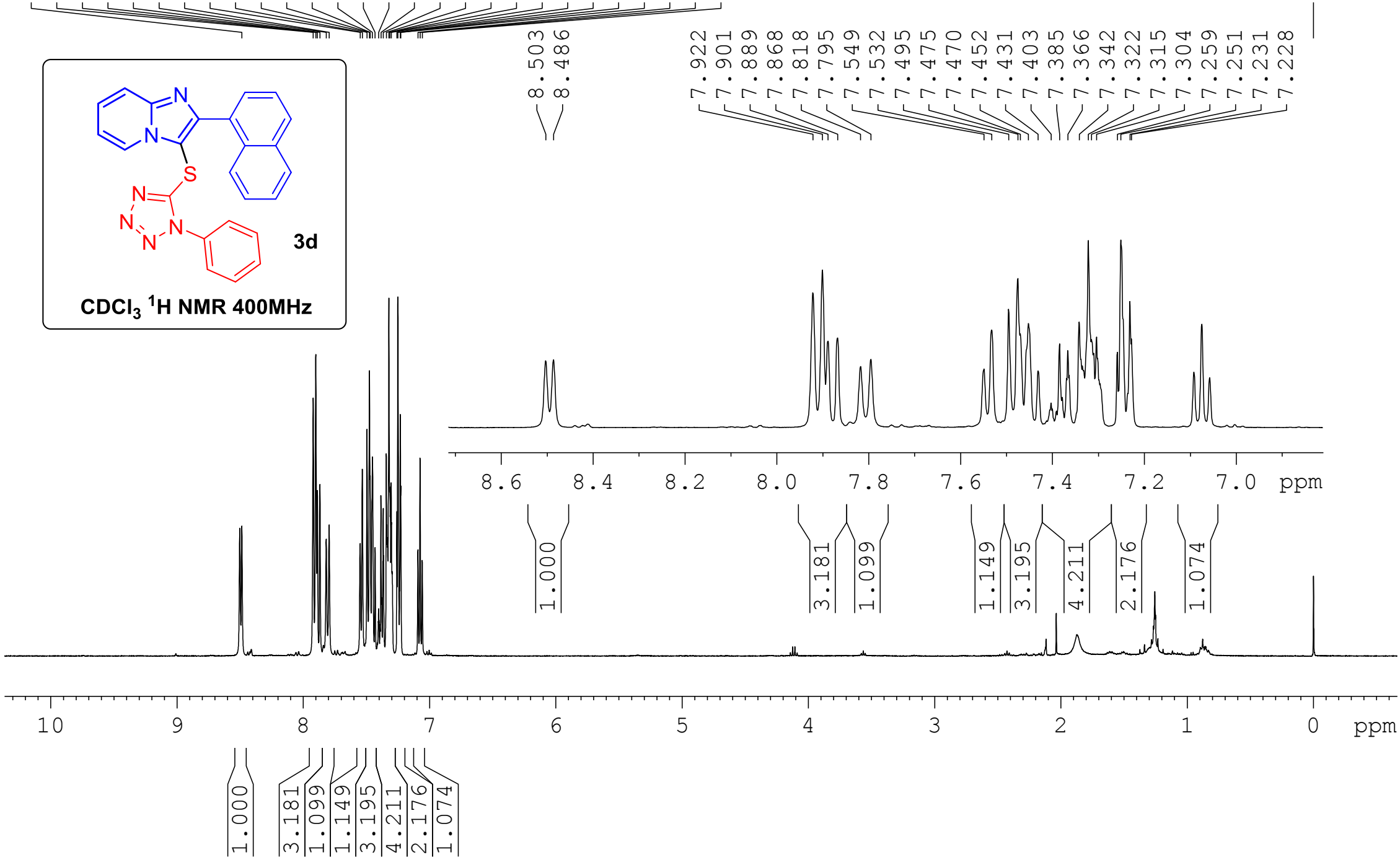




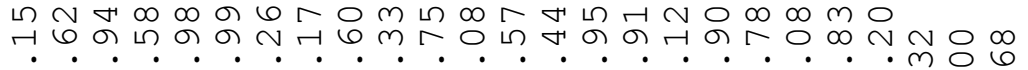

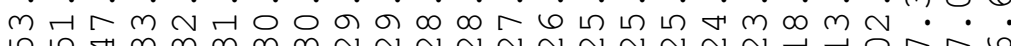

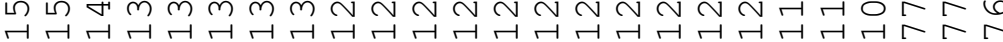

(n)

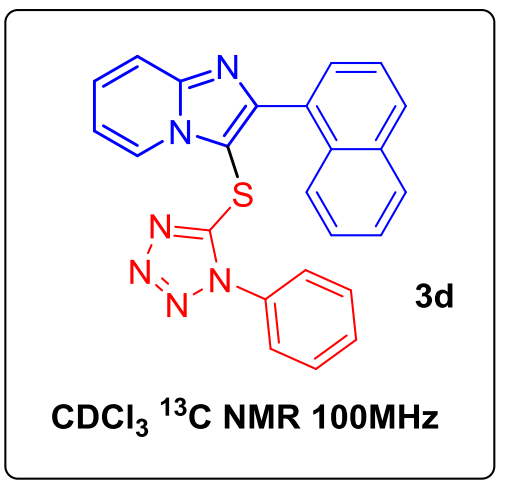

d

$\mathrm{CDCl}_{3}{ }^{13} \mathrm{C} \mathrm{NMR} 100 \mathrm{MHz}$

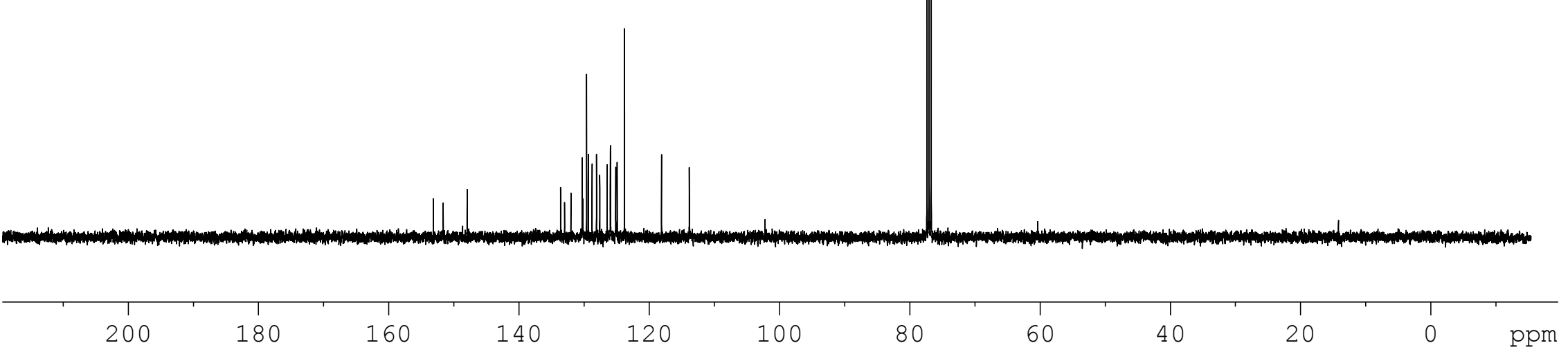




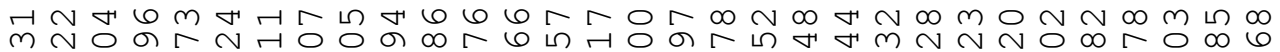

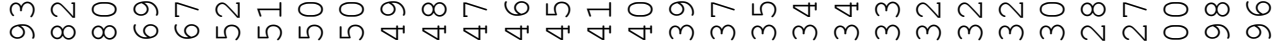

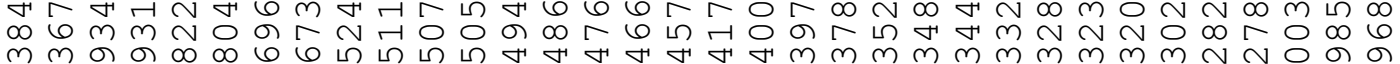

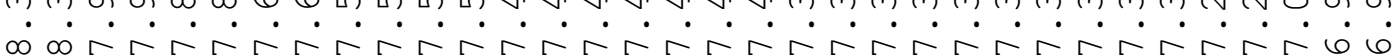

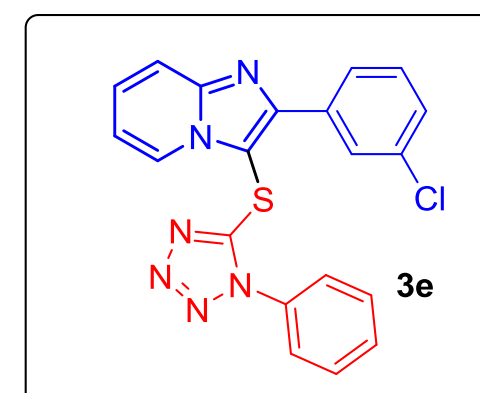

$1 \underbrace{\circ}_{1}$

$\mathrm{CDCl}_{3}{ }^{1} \mathrm{H}$ NMR $400 \mathrm{MHz}$

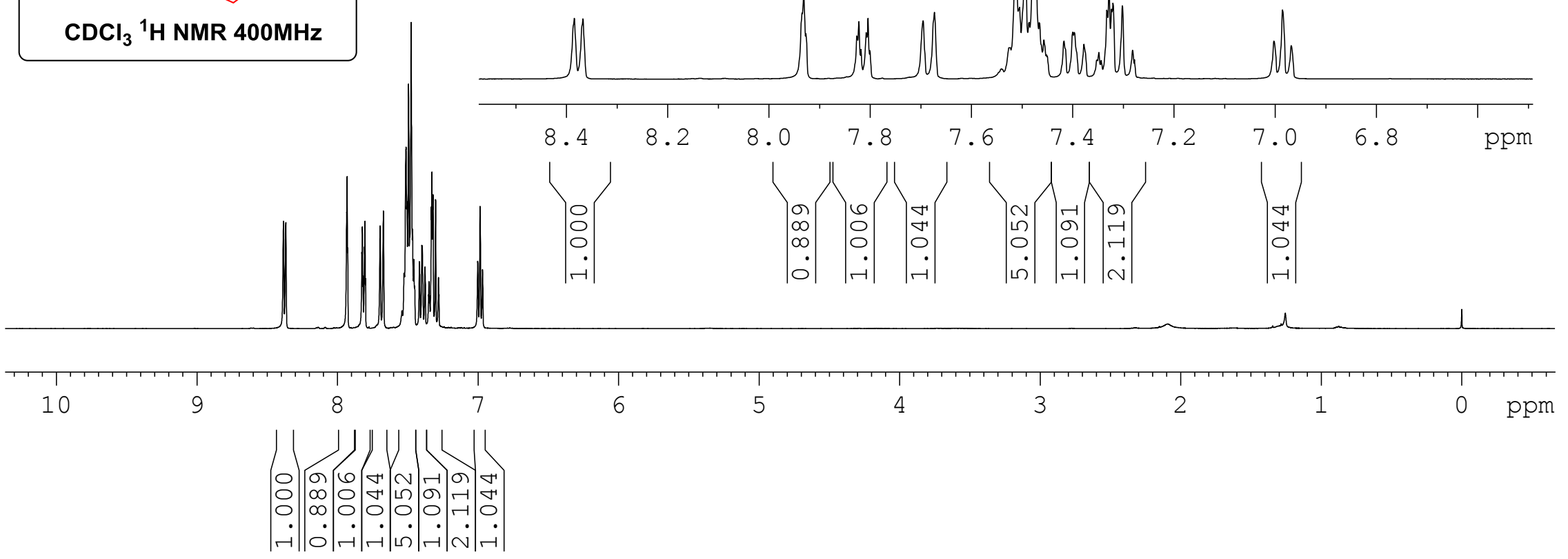


の

0. เी

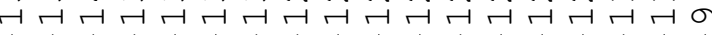

$\longrightarrow$

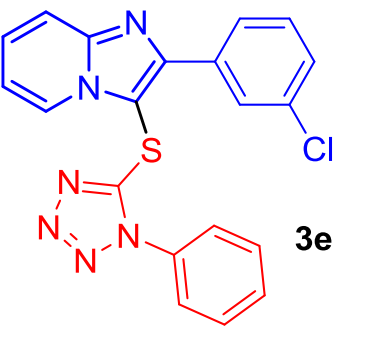

$\mathrm{CDCl}_{3}{ }^{13} \mathrm{C}$ NMR $100 \mathrm{MHz}$

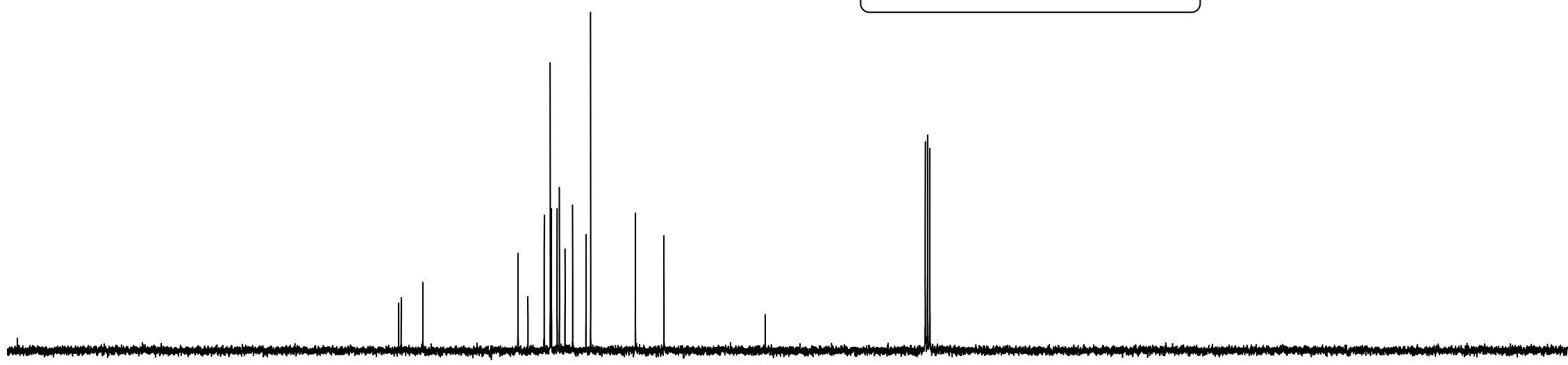

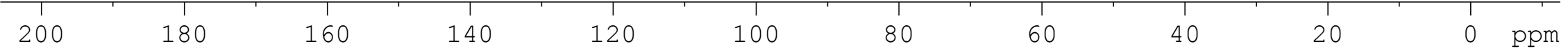


응유

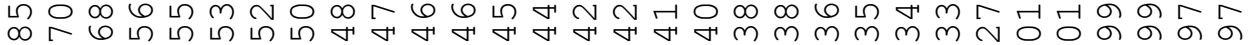

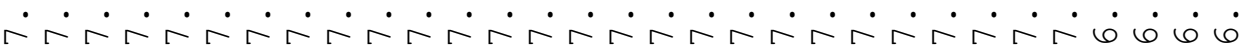

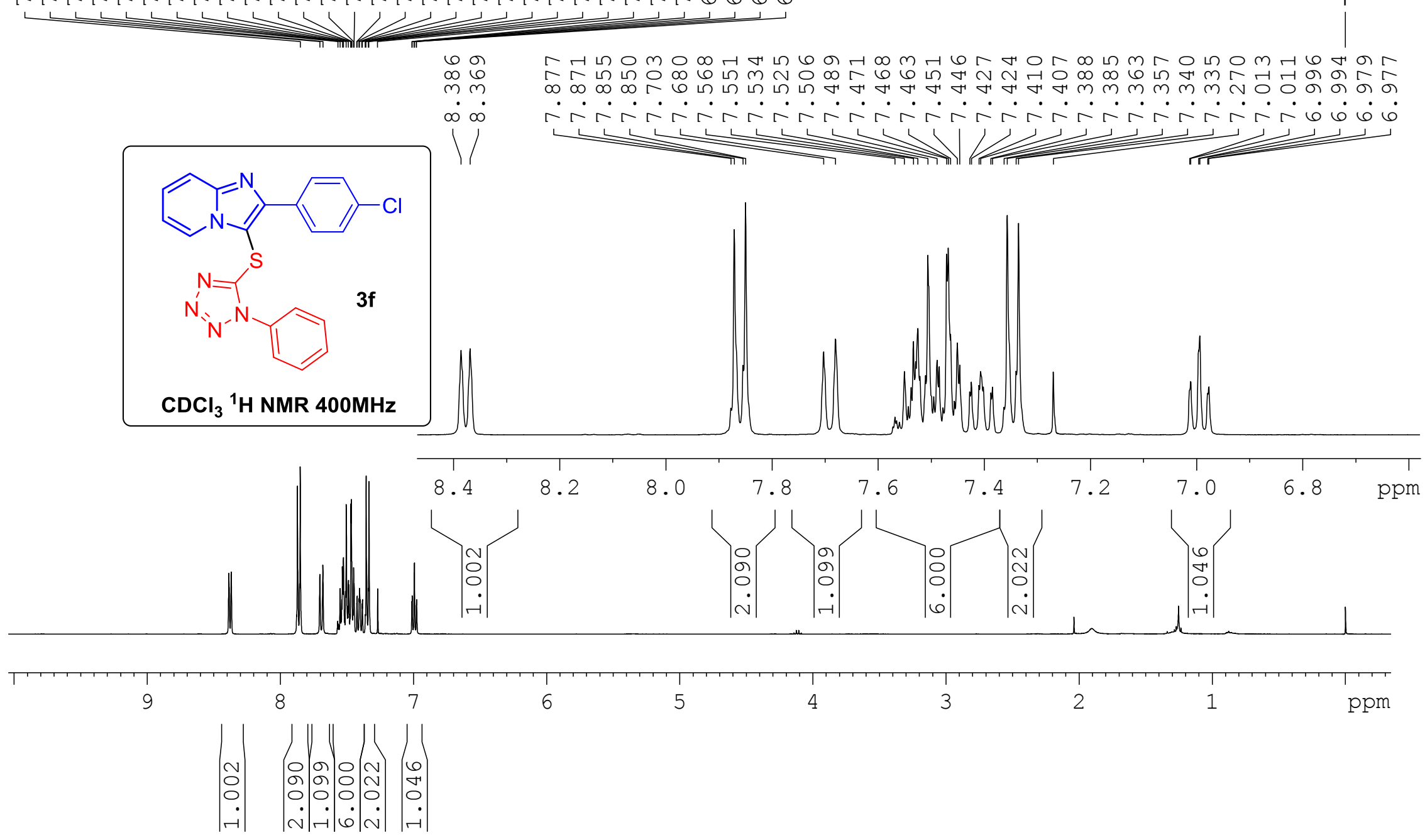




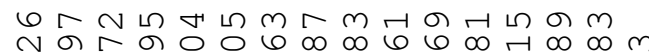

เூ

(

$\stackrel{n}{\infty} \circ 0$

$\therefore$

ㄷำ

Y
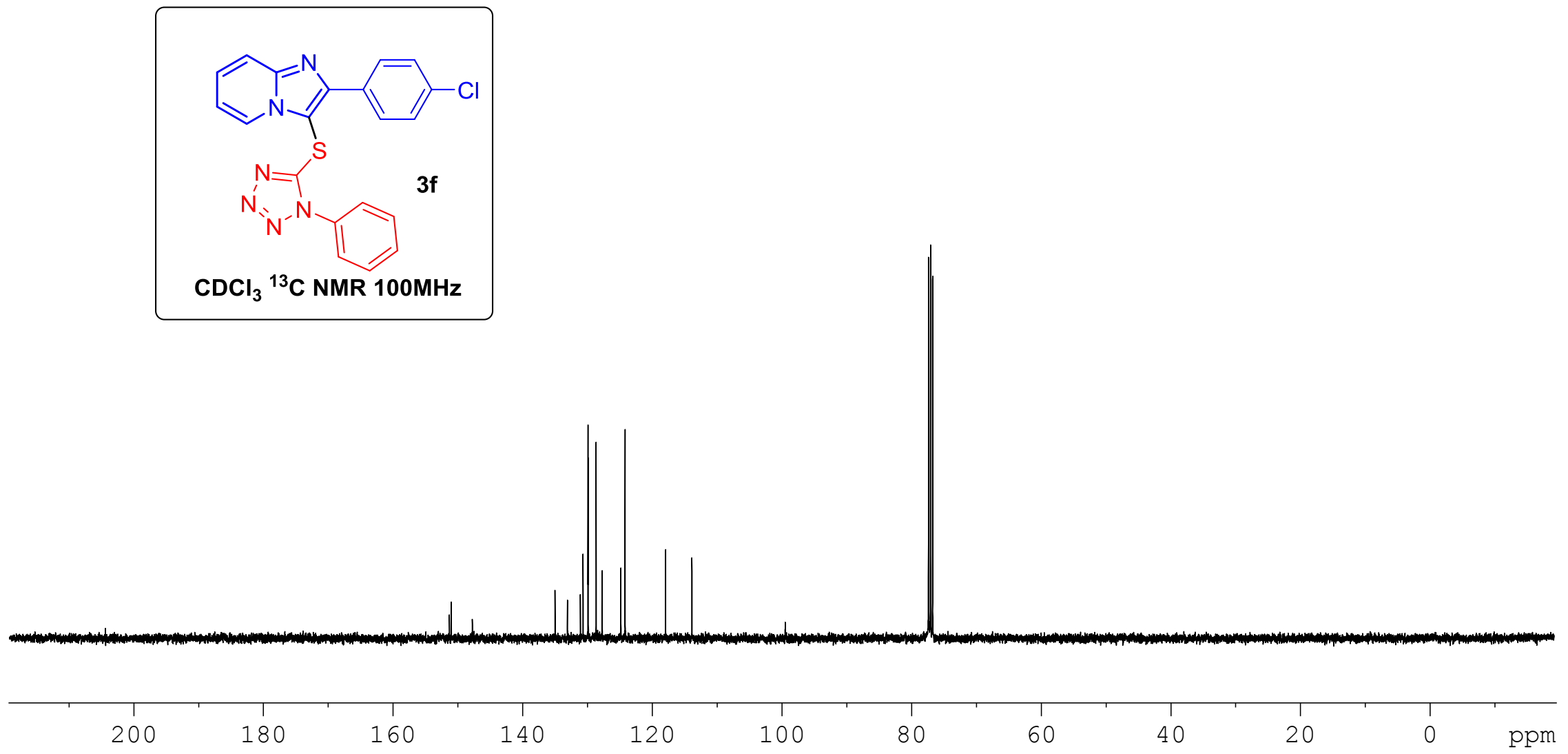
HN요

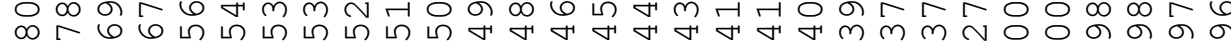

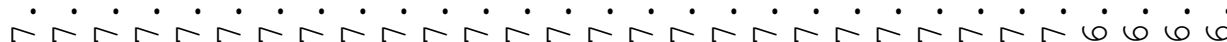

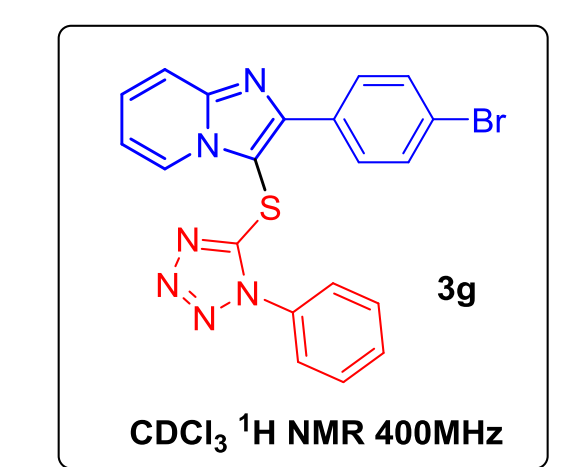

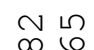

HNNOHGGmamLG $\infty m$

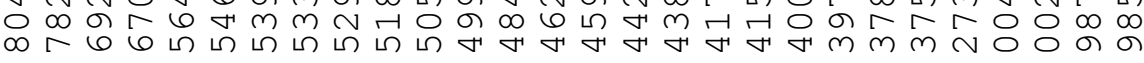

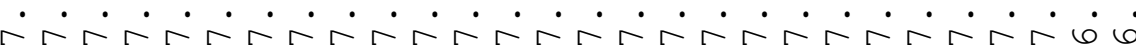
$1 /$
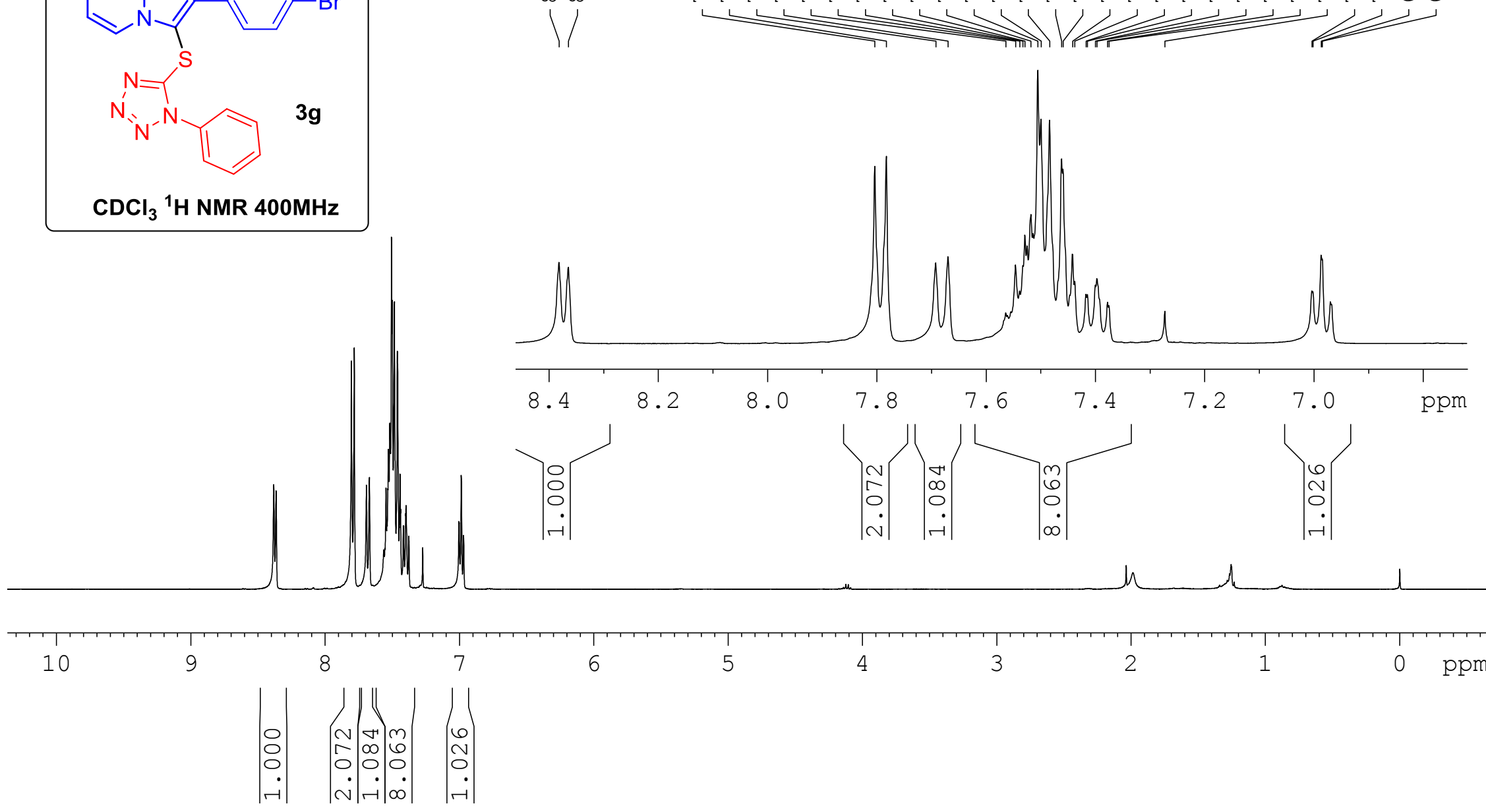


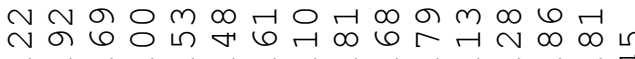

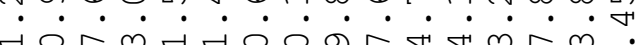

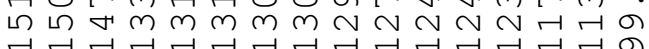

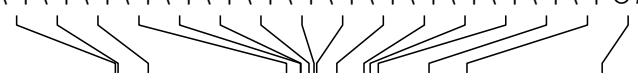

mo 00

둥

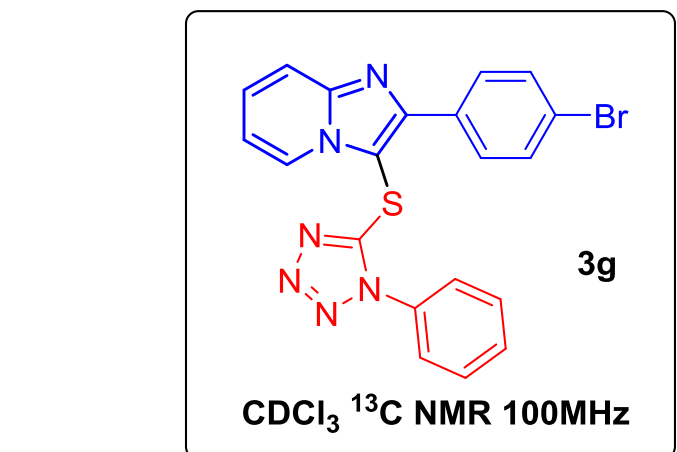

$\mathrm{CDCl}_{3}{ }^{13} \mathrm{C}$ NMR $100 \mathrm{MHz}$

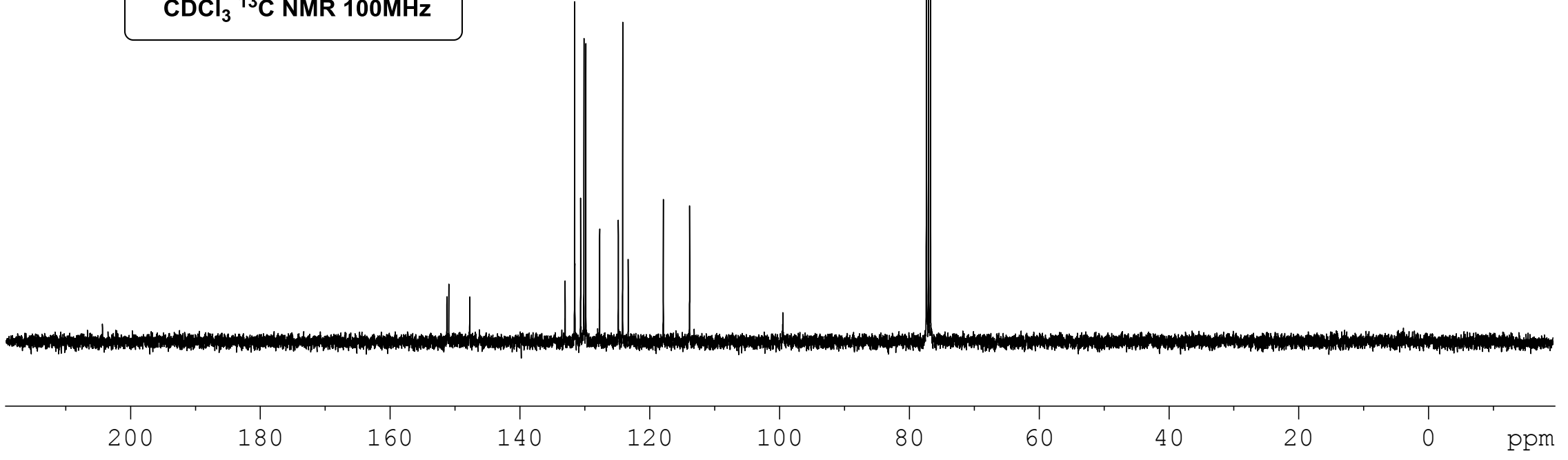




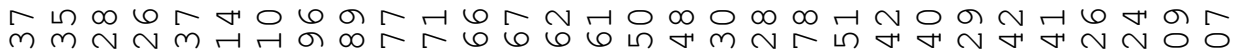

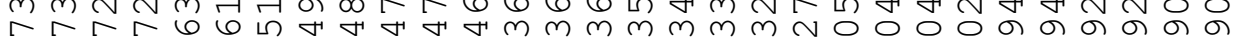

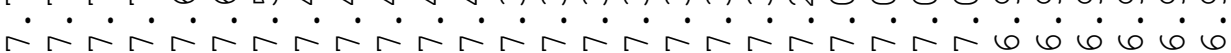

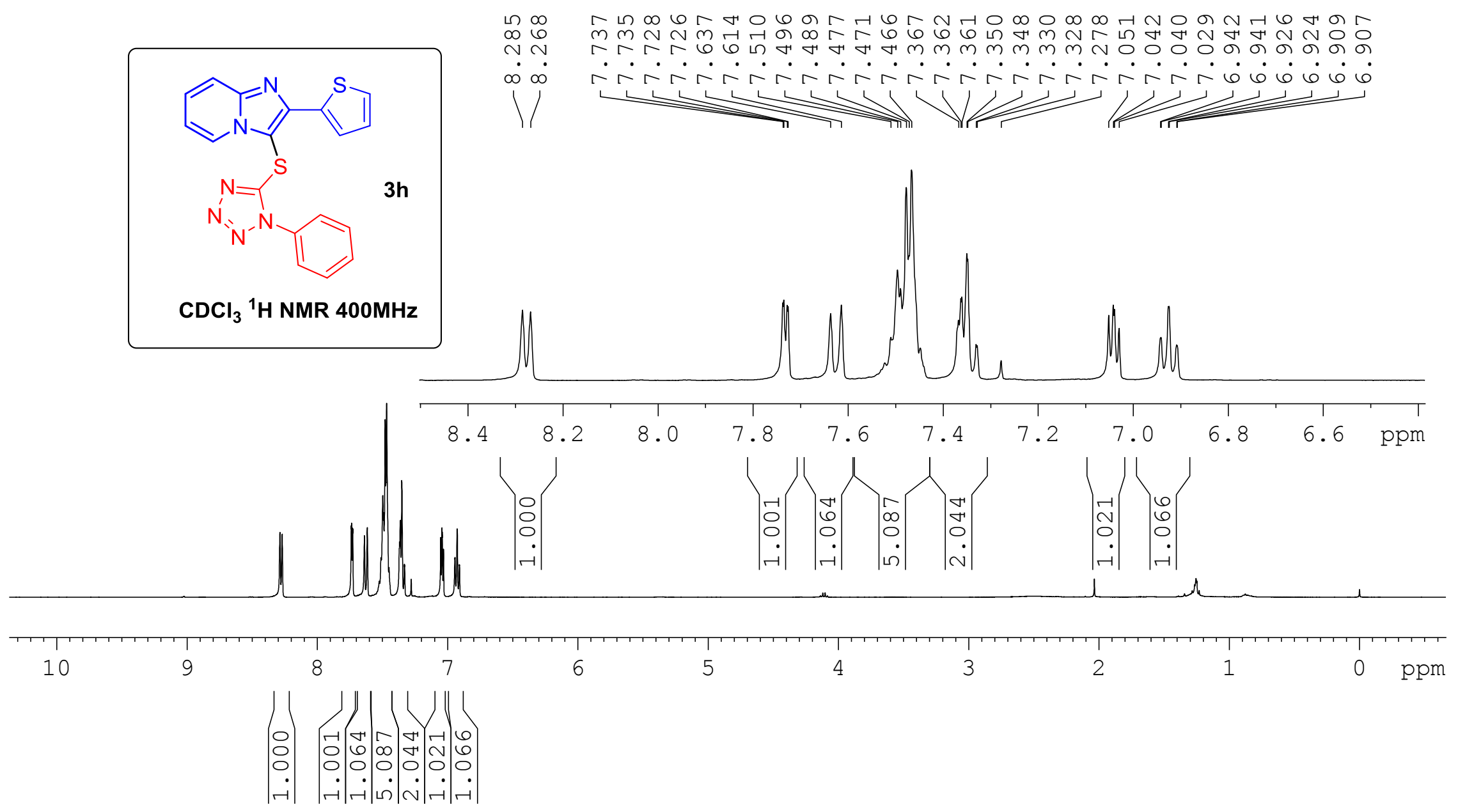




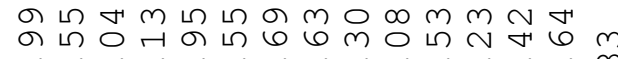

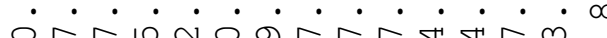

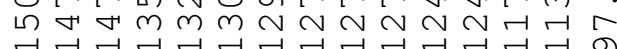

11

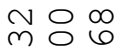

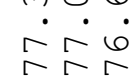
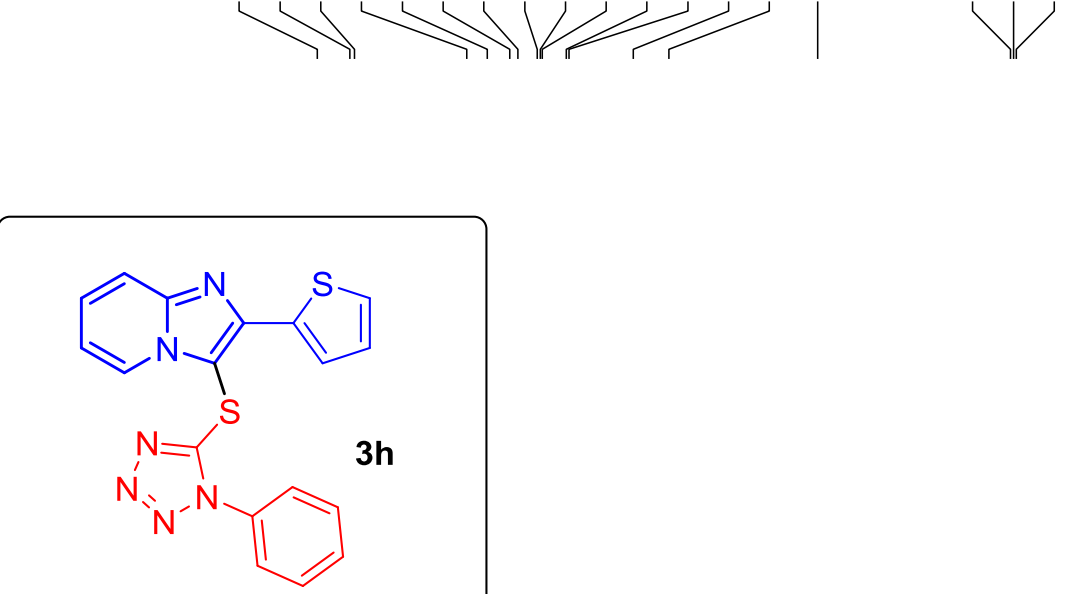

$\mathrm{CDCl}_{3}{ }^{13} \mathrm{C}$ NMR $100 \mathrm{MHz}$

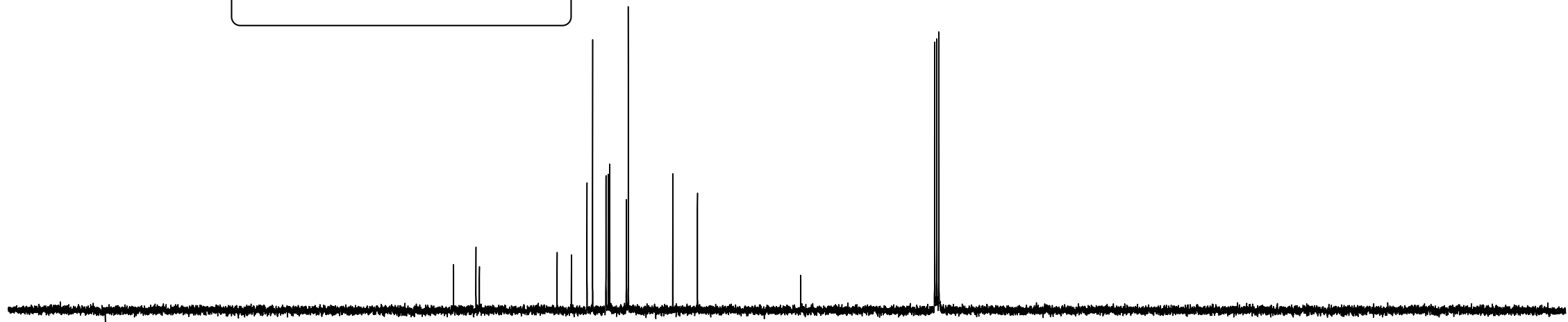


の

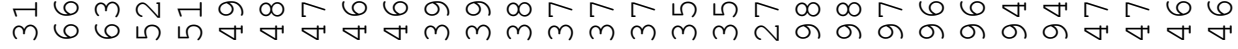

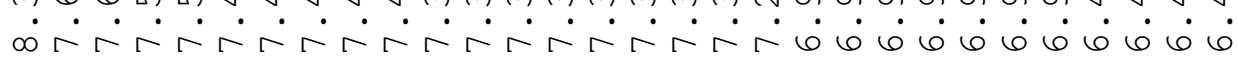

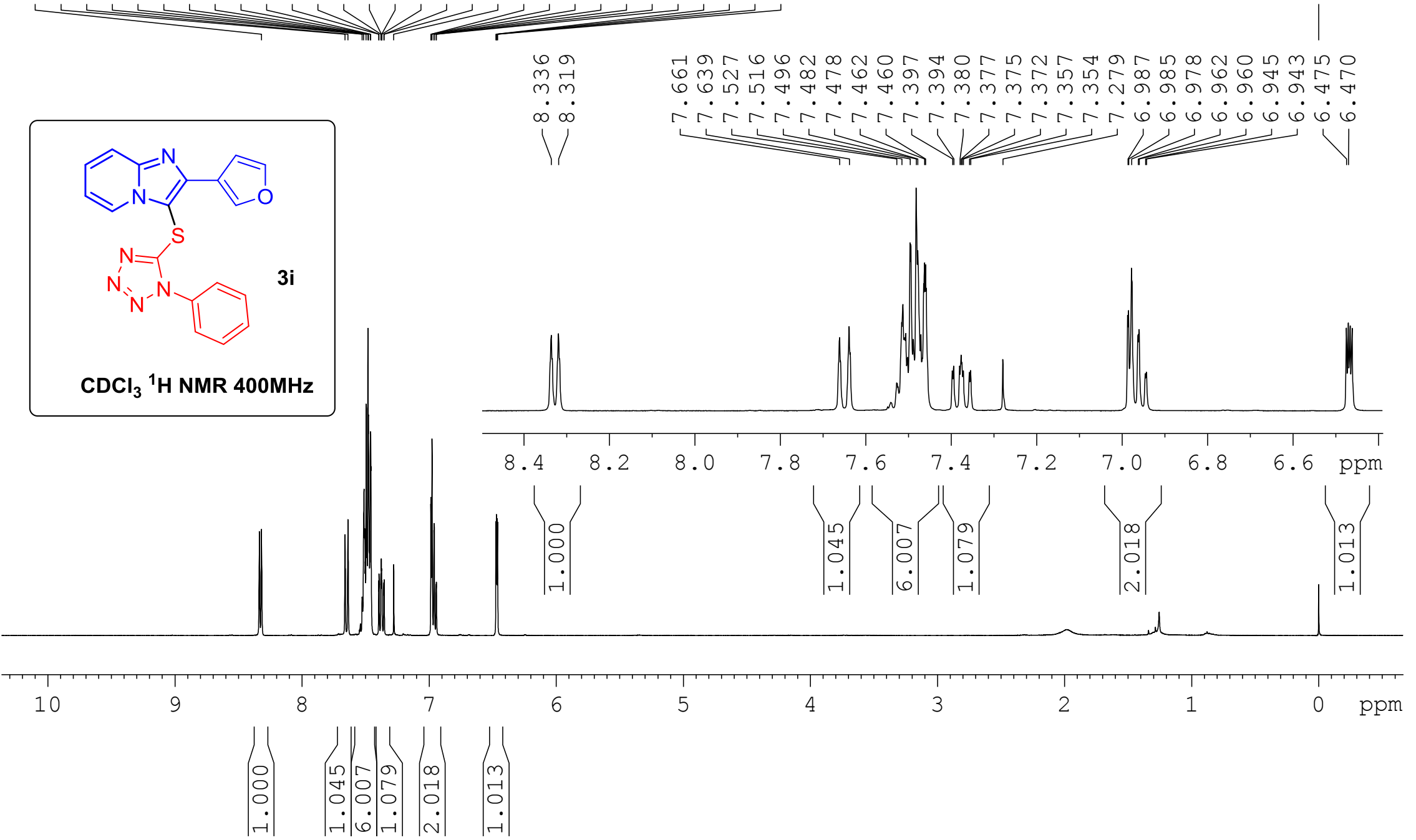




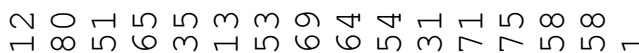

- $\dot{0} \dot{0} \dot{0} \dot{0} \dot{0} \cdot \dot{m}$

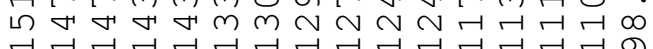

$\rightarrow$

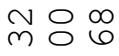

둥

V

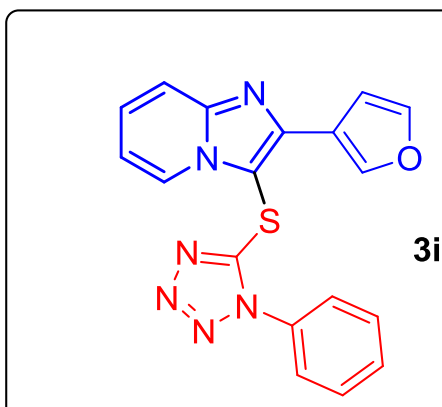

$\mathrm{CDCl}_{3}{ }^{13} \mathrm{C}$ NMR $100 \mathrm{MHz}$

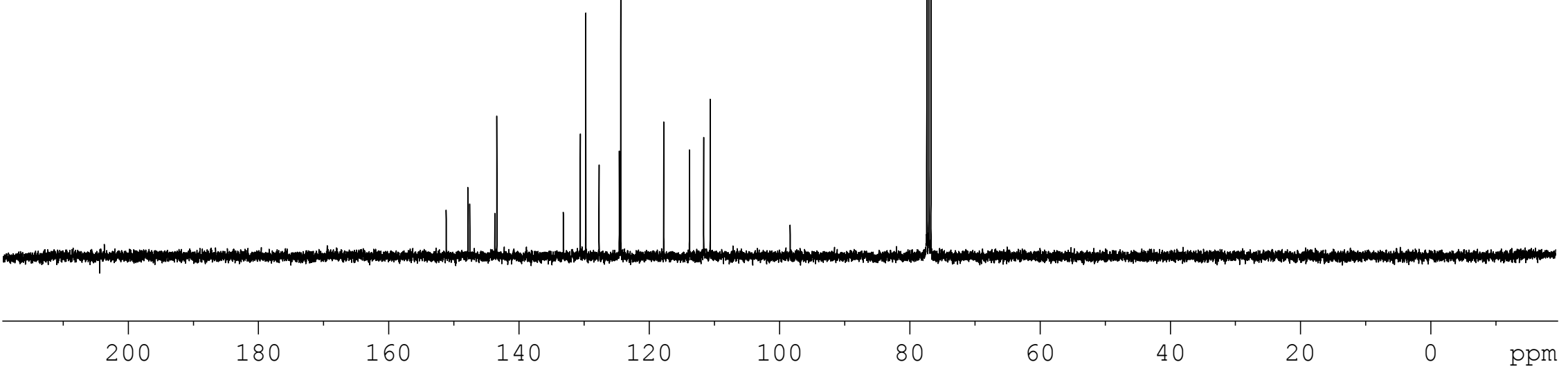




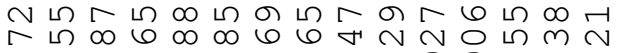
H $\dot{0} \dot{0} \dot{0} \dot{0} \dot{\circ} \dot{0} \dot{0} \dot{0} \dot{0}$

$3<1311$
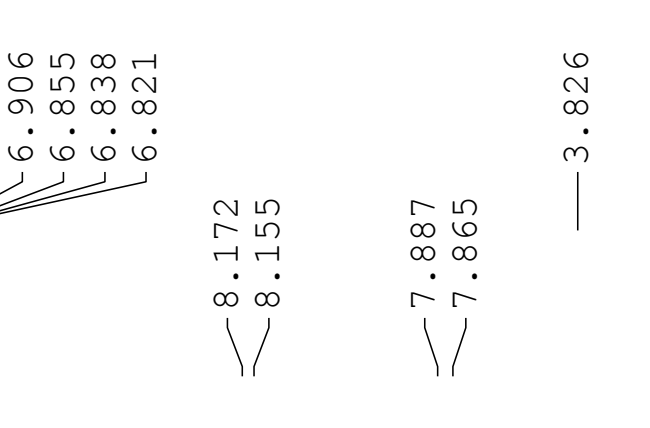

$\mathrm{CDCl}_{3}{ }^{1} \mathrm{H}$ NMR $400 \mathrm{MHz}$
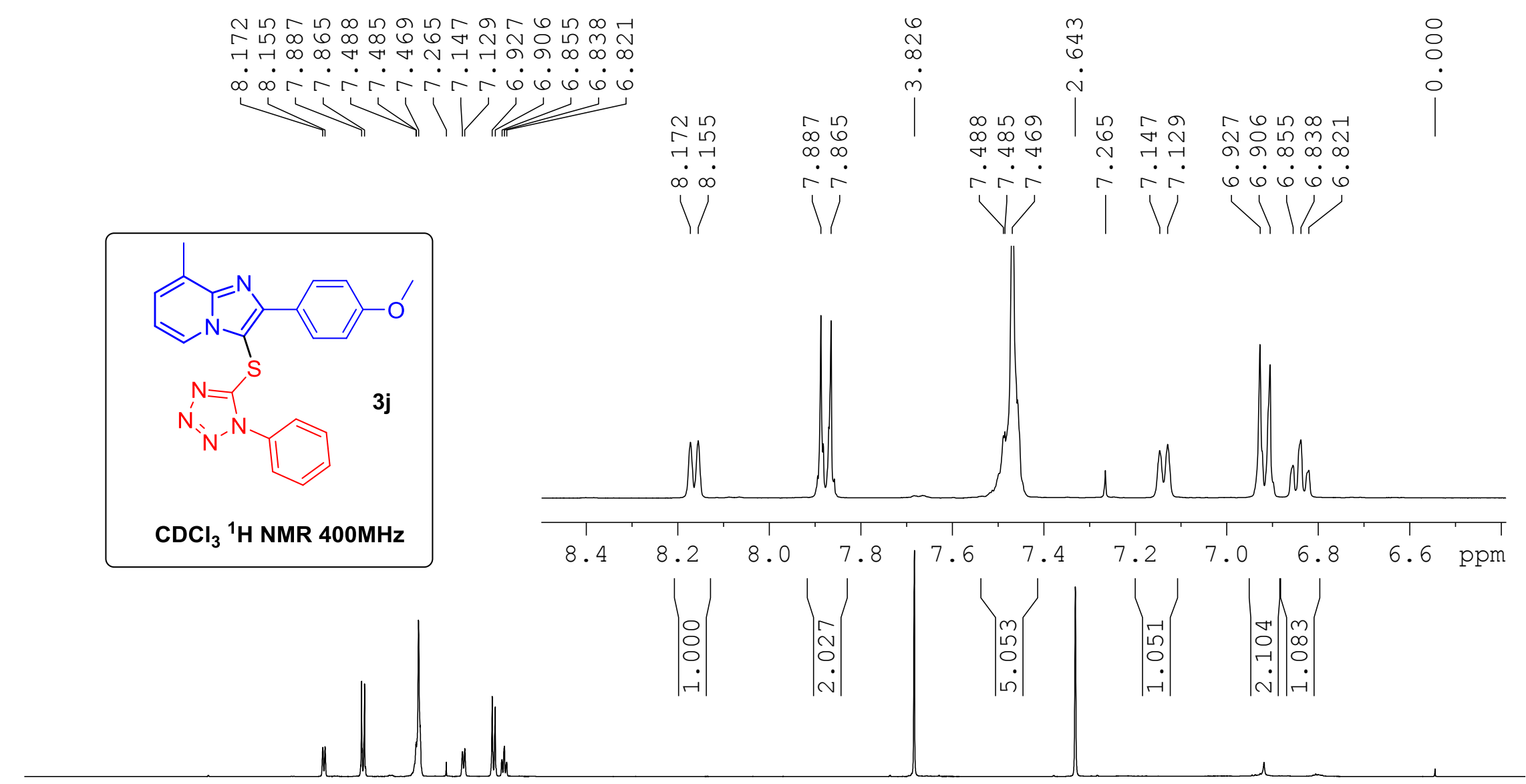

$m$
$\stackrel{m}{+}$
$\dot{v}$
$\dot{v}$

$\circ$
8
0
0
1

ก)

ㄱ. ㄷ. न न $\infty \infty_{\infty} \infty$

- $\dot{1} \dot{0} \dot{0} \dot{0}$

$1 / 11$

$11 /$

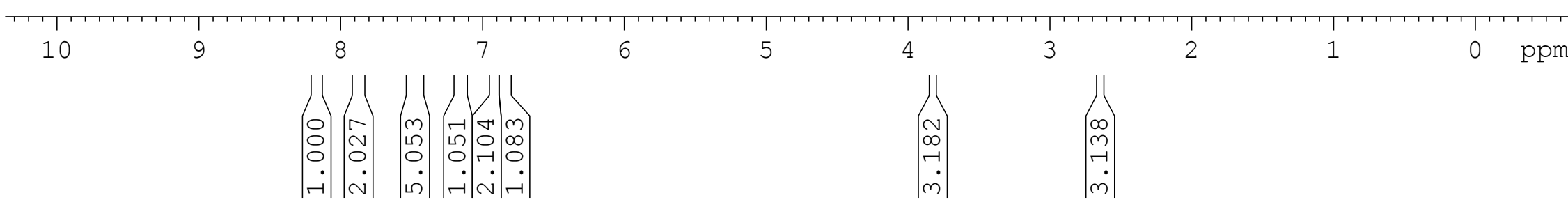


긍

$0-1$ mo 0 ñ

$b$ 光

111

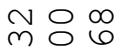

$\therefore \therefore \dot{0}$

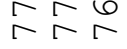

$Y$

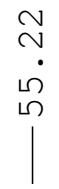

$\tilde{6}$
6
+

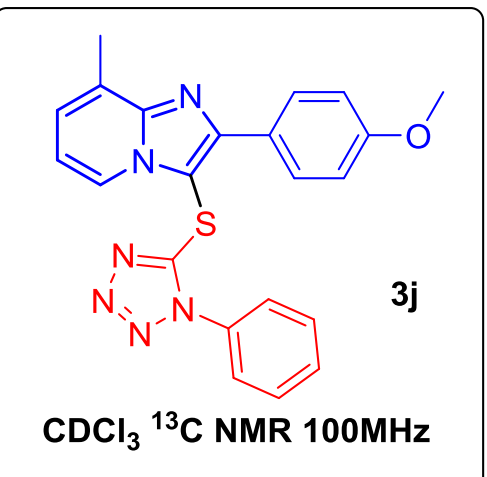

$\mathrm{CDCl}_{3}{ }^{13} \mathrm{C}$ NMR $100 \mathrm{MHz}$

180

160

140

120

100

80

60

20

ppm 


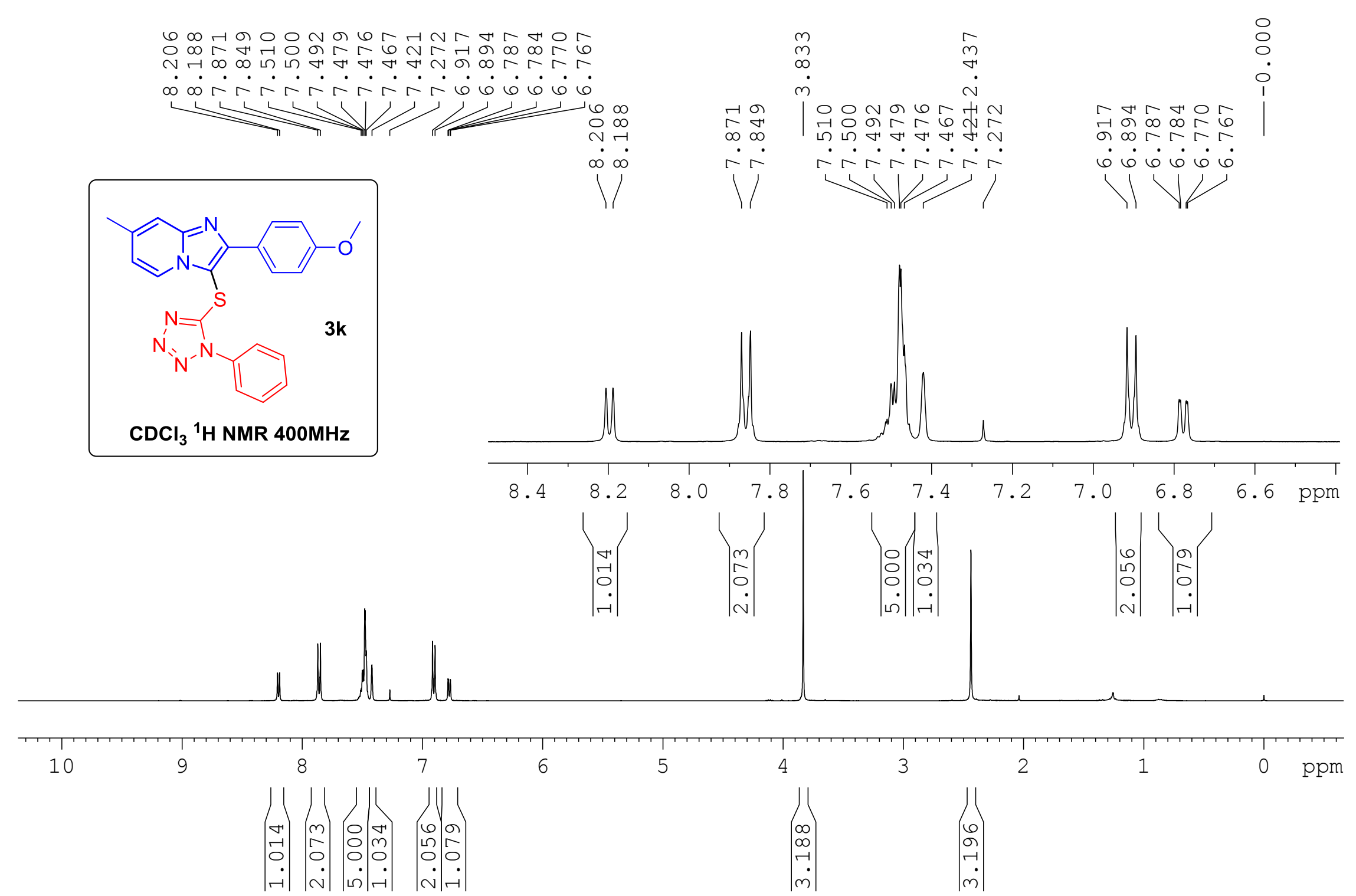




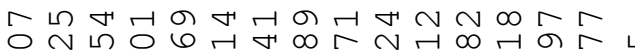

$\dot{0} \dot{\infty} \dot{0} \dot{0} \dot{0} \dot{0} \dot{m}$

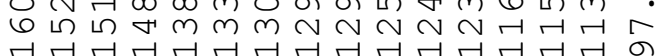

।
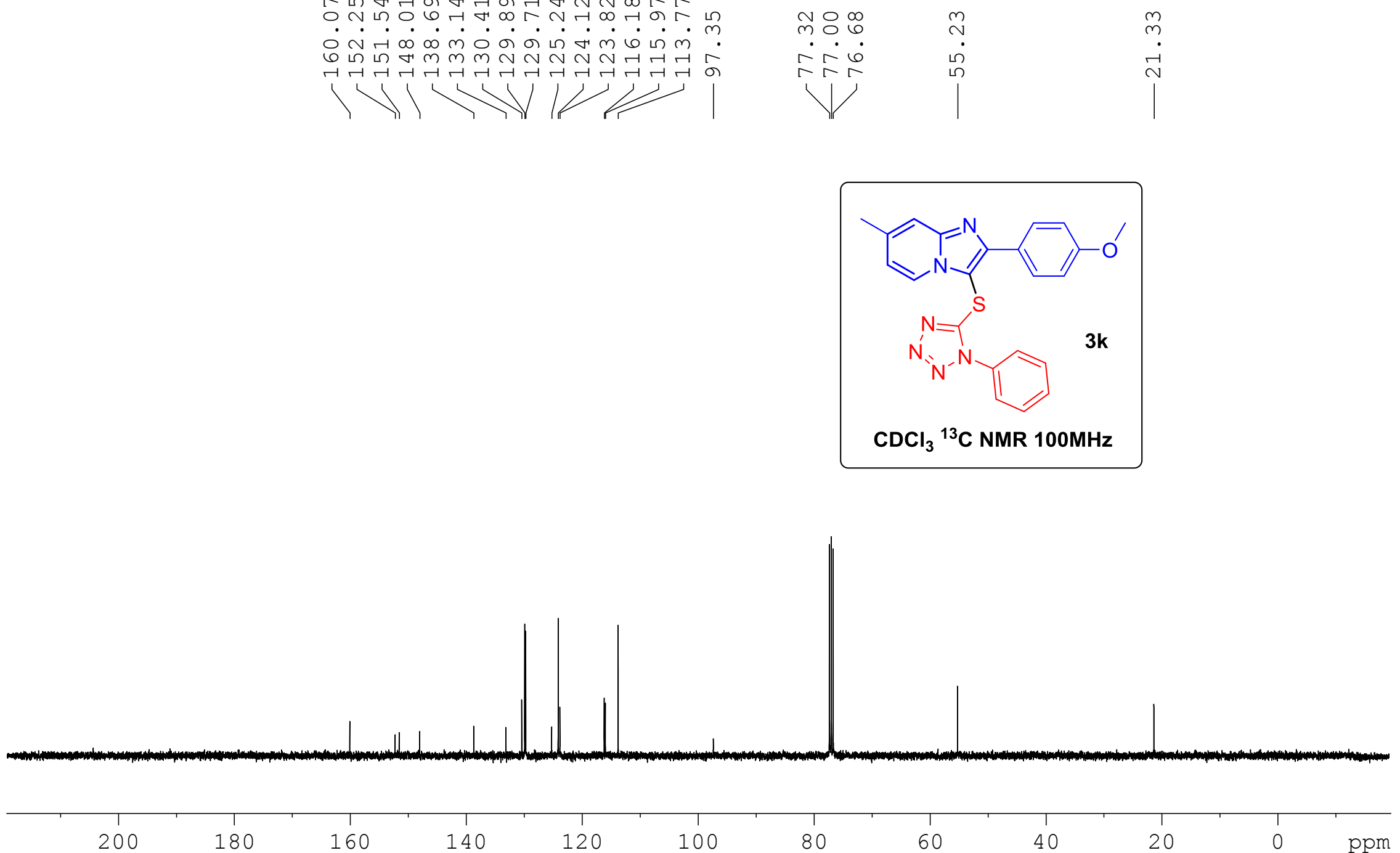


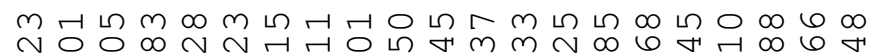
웃

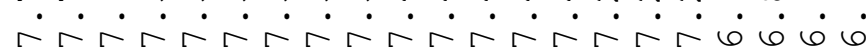

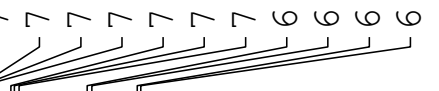

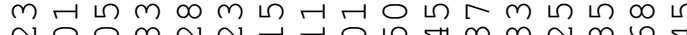

$N O O \infty N N$ H

. . . . . . . . . . . . . .

$\mathrm{S}^{\mathrm{N}}$

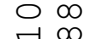

ने $\infty$

$\dot{0}$

11

66

66

1
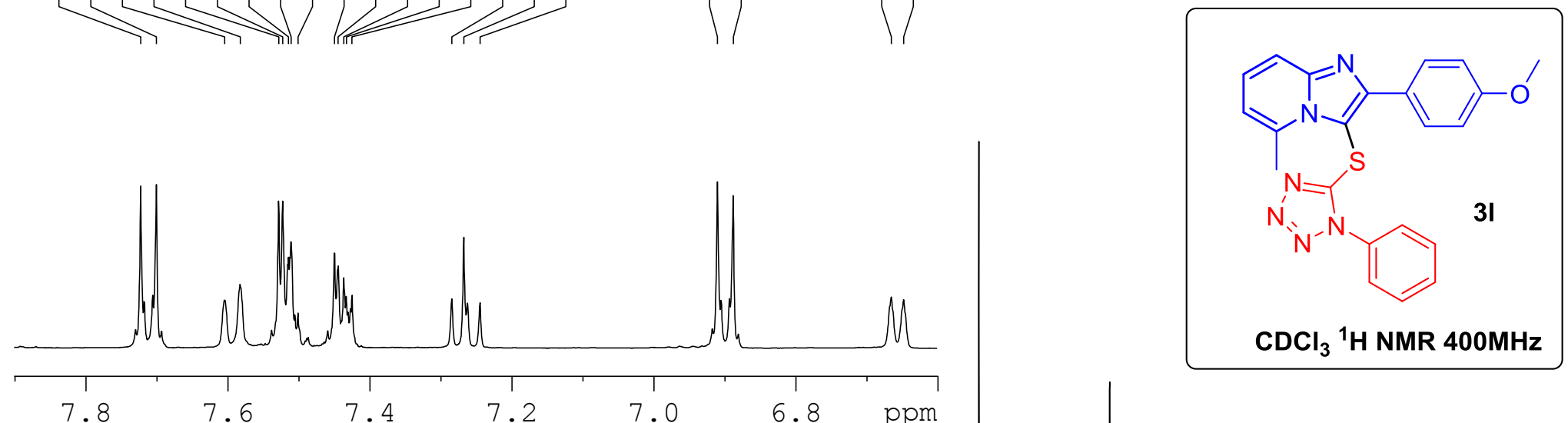

7.8
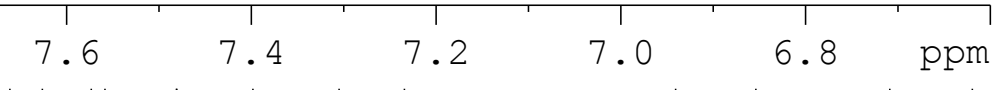

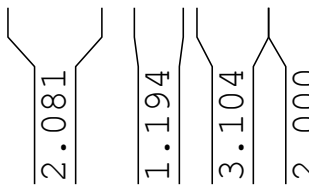
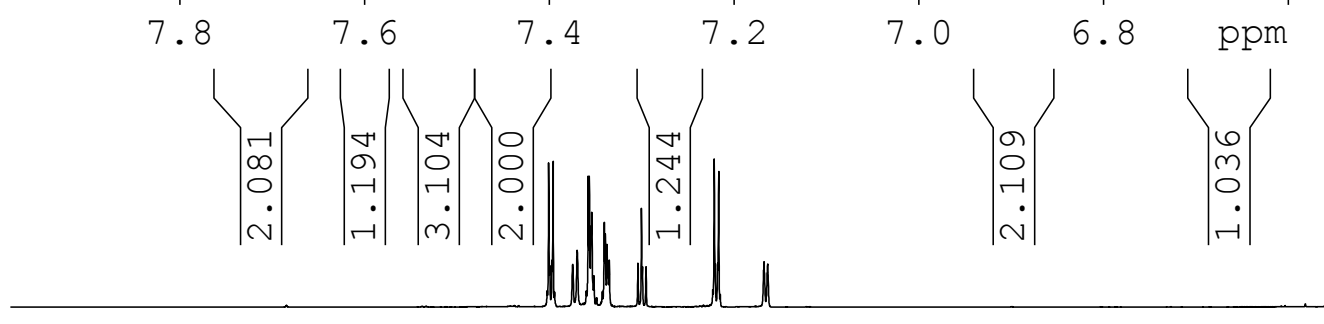

\begin{tabular}{ll}
\multicolumn{1}{r}{} & \multicolumn{2}{c}{} \\
$\infty$ & ने \\
$\dot{m}$ & $\dot{\sim}$
\end{tabular}

0
$\circ$
0
0
1

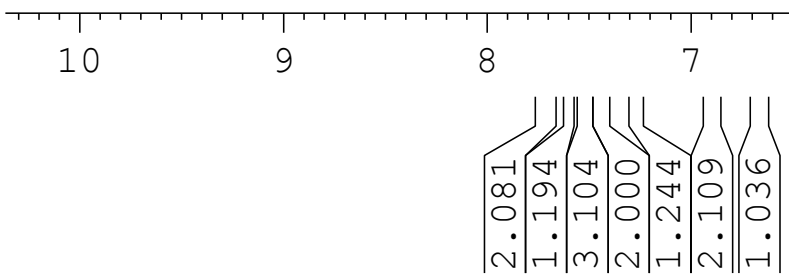

6

5
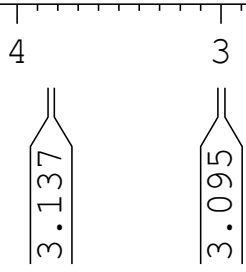

2

1

0 ppm

$\left|\begin{array}{c}n \\ 0 \\ 0 \\ \dot{m}\end{array}\right|$ 
Na

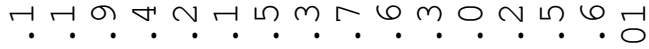
0 अ 年

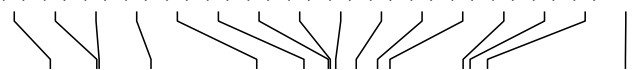
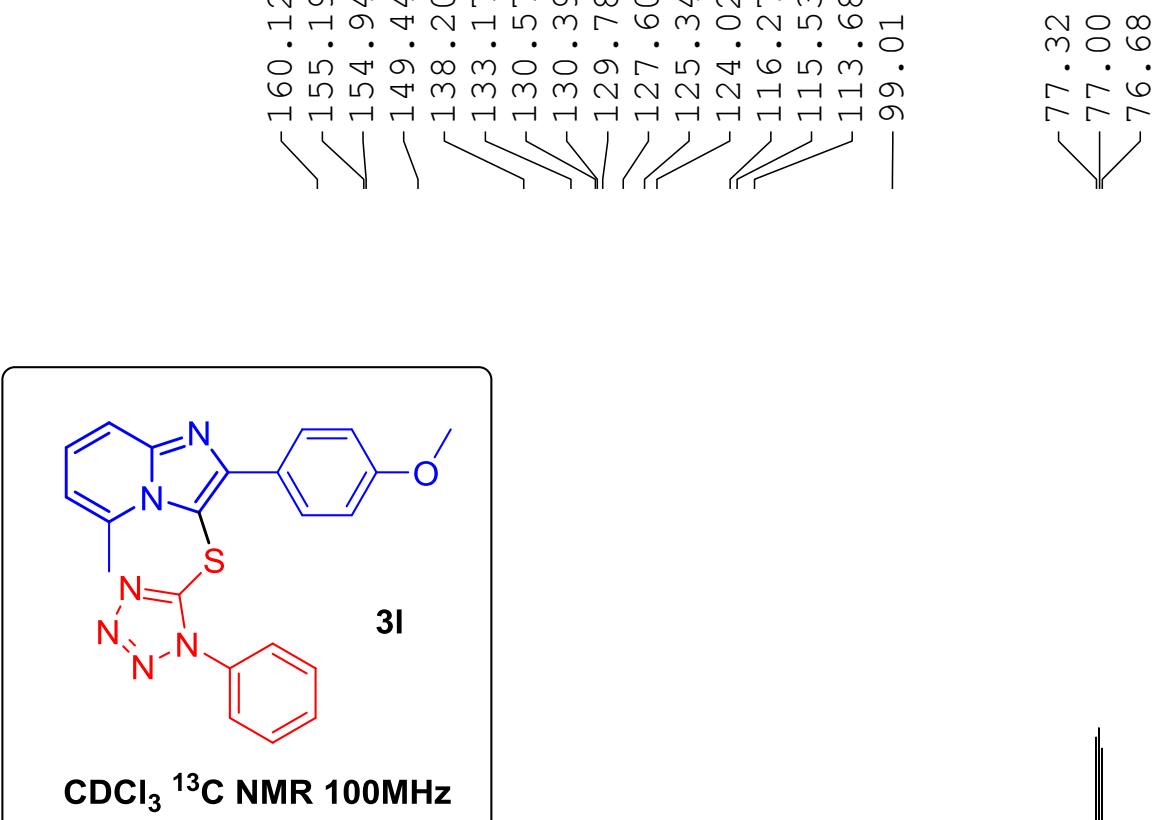

C NMR 100MHz

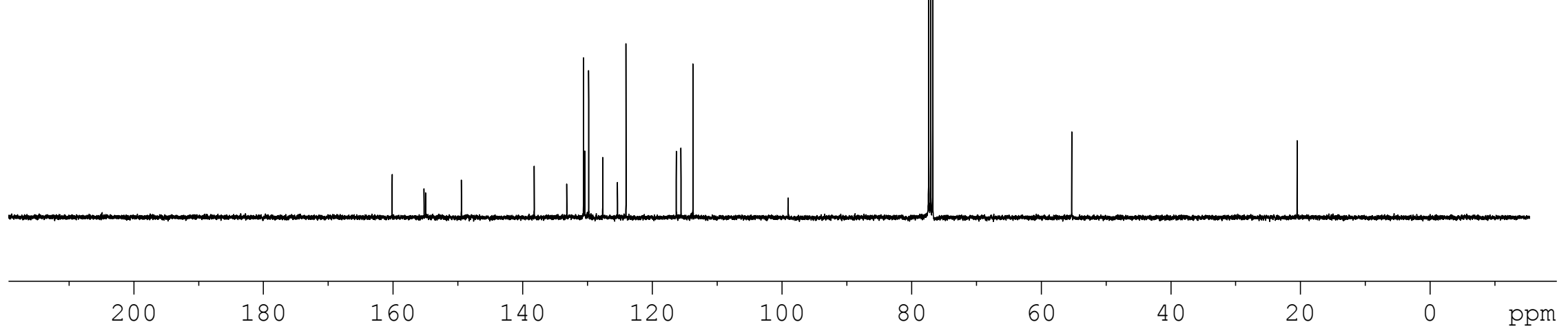




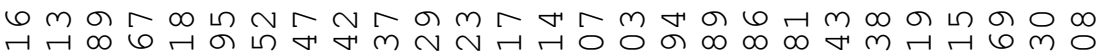

$\infty 0^{-}-100$

- $6 m$ m

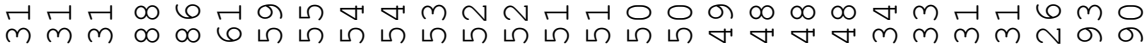

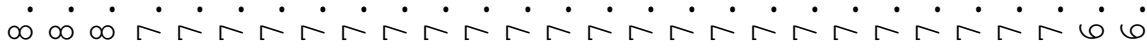

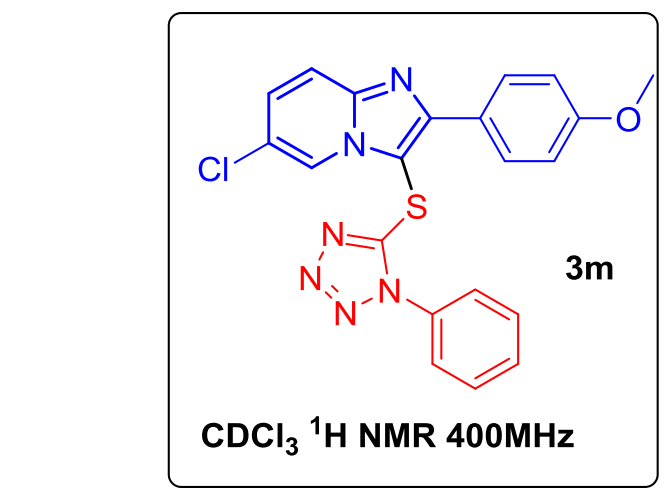

12
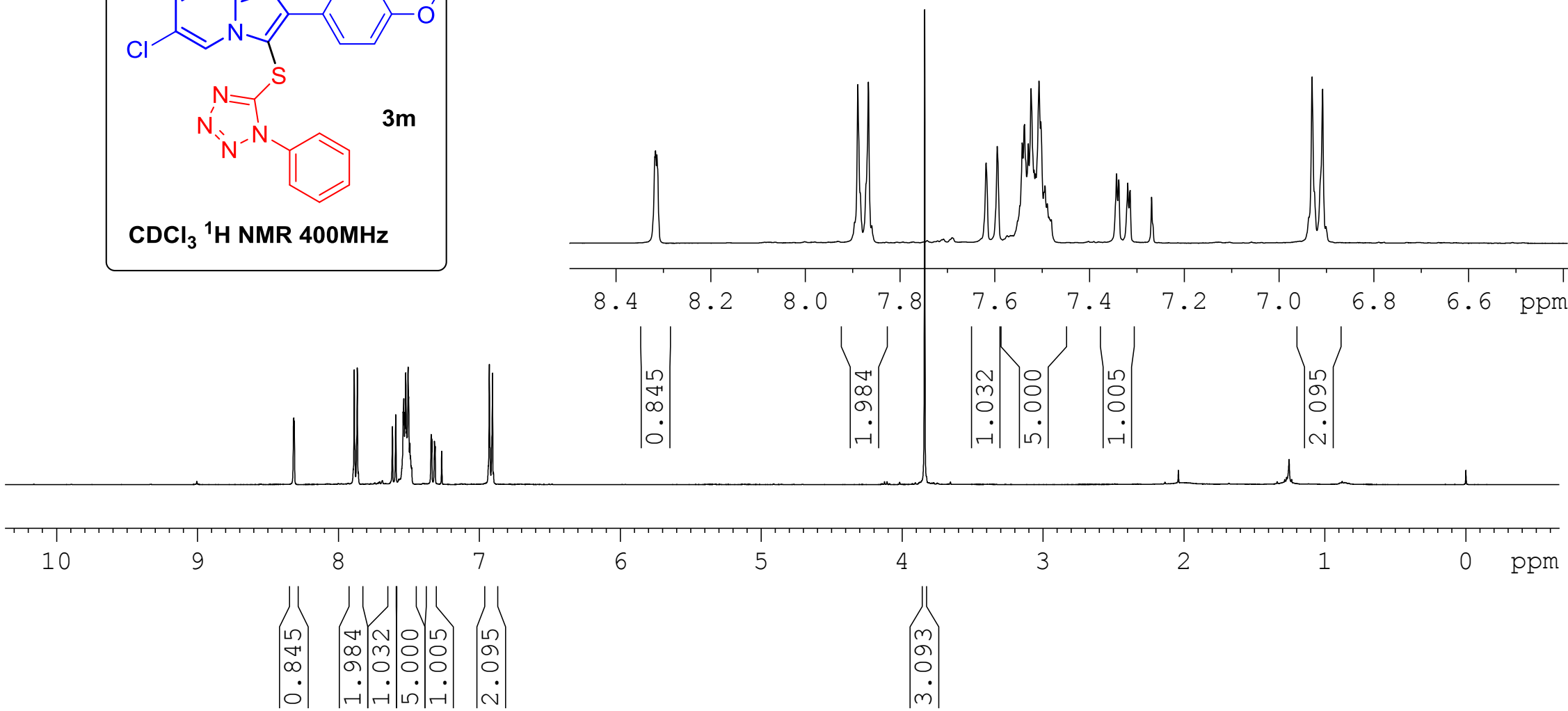


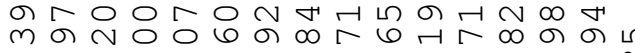

$\circ \sim-100$

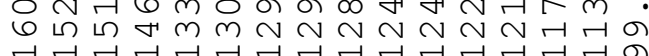

111
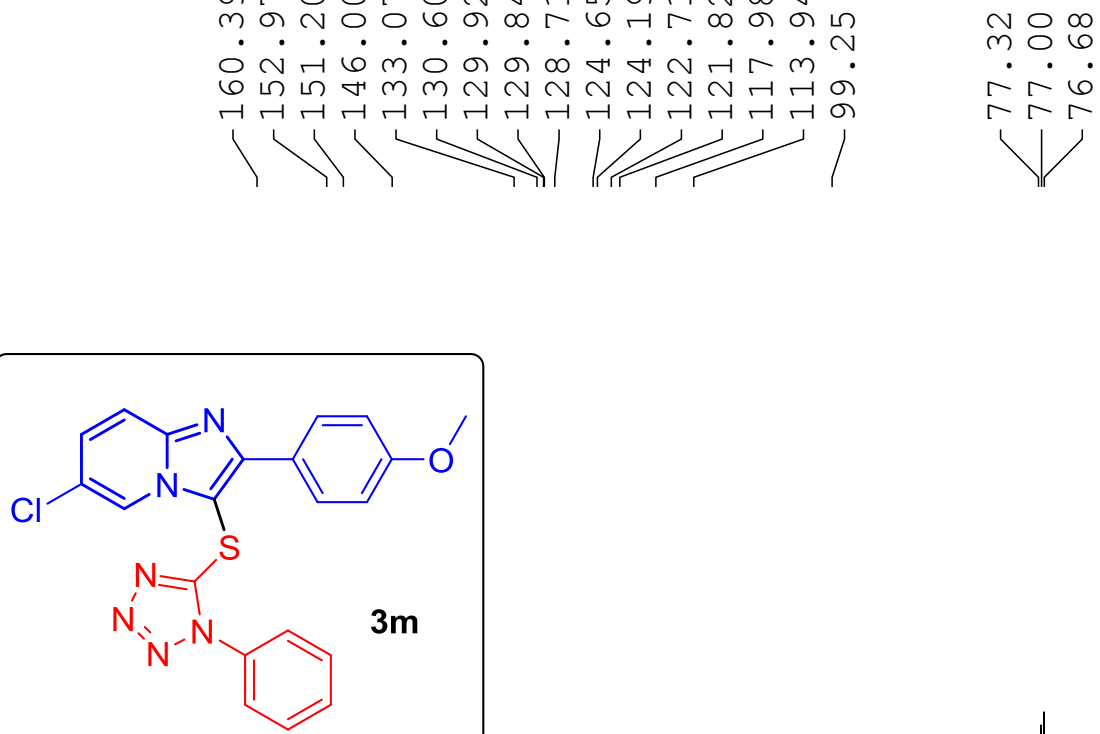

$\mathrm{CDCl}_{3}{ }^{13} \mathrm{C}$ NMR $100 \mathrm{MHz}$

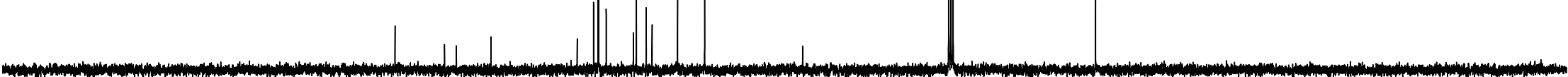


강

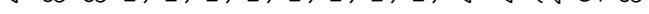

$\dot{0} \cdot \dot{0} \cdot \dot{0} \cdot \dot{0} \cdot \dot{0}$
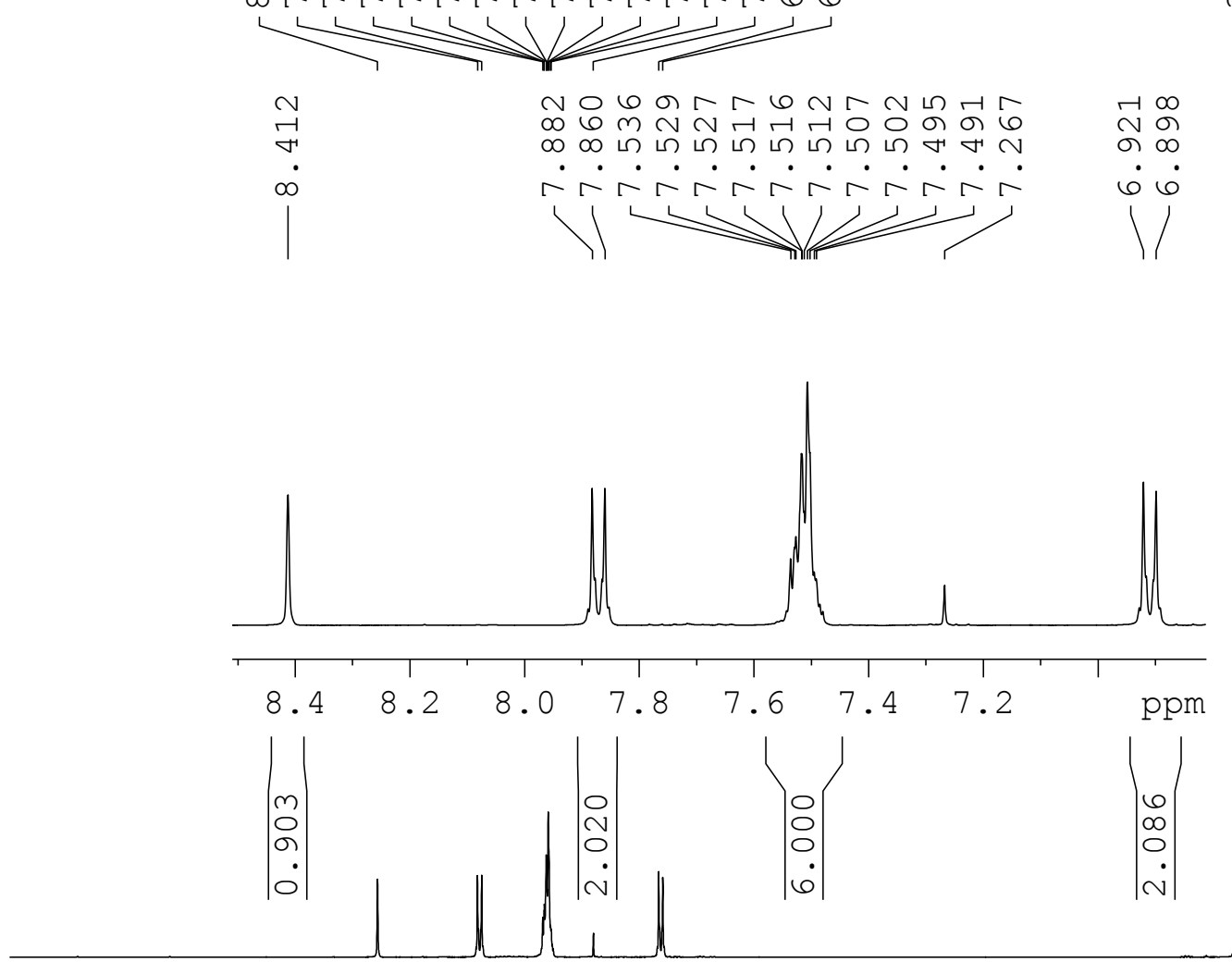

$\stackrel{6}{m}$

$\infty$
$\infty$
$\forall$

i

ᄋ
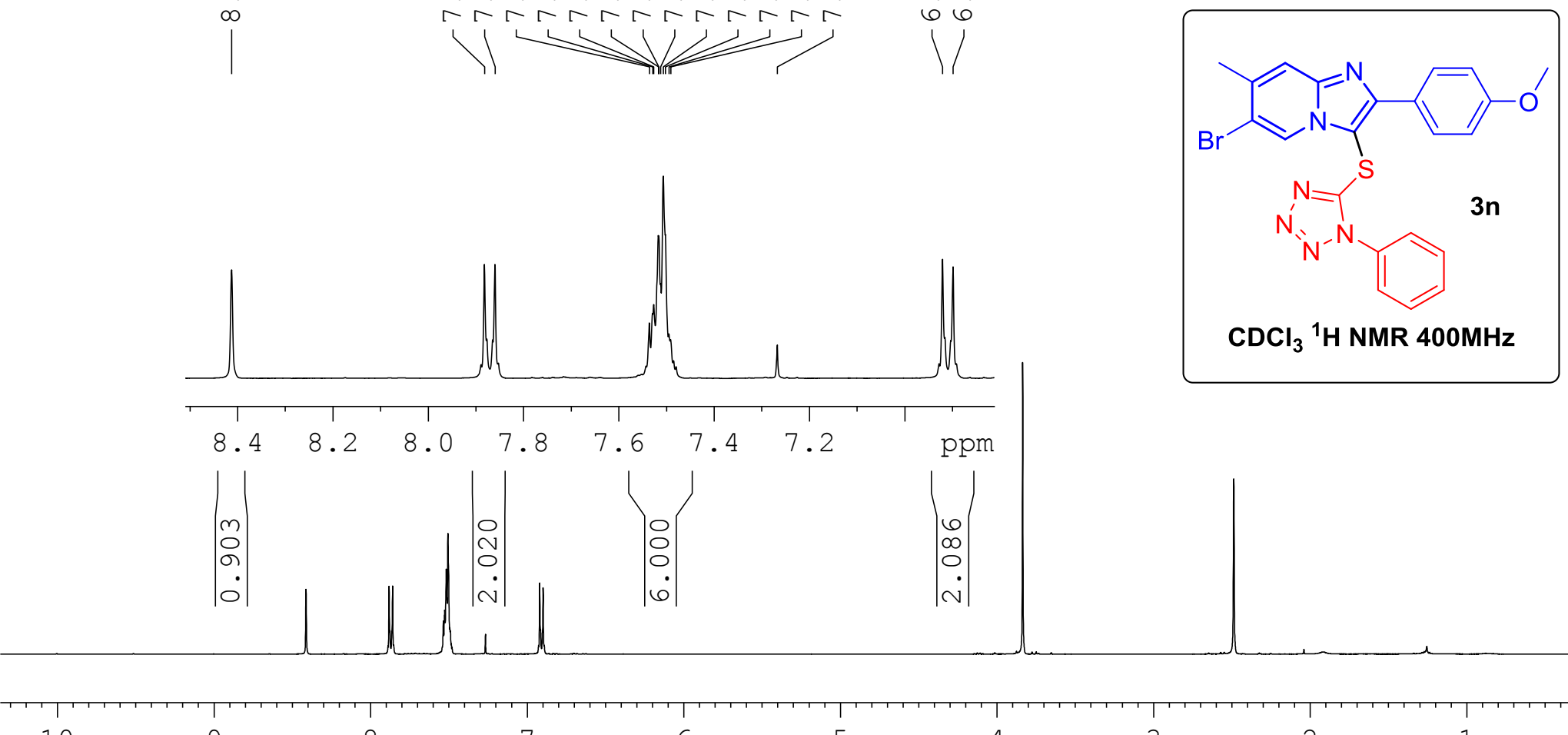

9

8

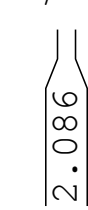

5

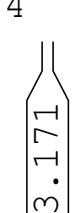

1

0 ppm

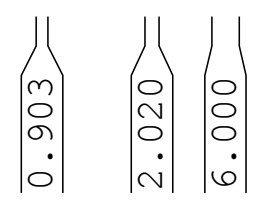

$\left|\begin{array}{c}1 \\ 6 \\ \infty \\ 0 \\ ن \\ \sim\end{array}\right|$

6

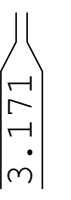

$\left|\begin{array}{c}n \\ \sigma \\ 0 \\ \dot{m}\end{array}\right|$ 


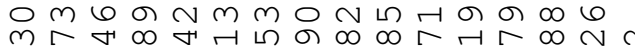

0 bำ 111

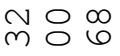 $\therefore \therefore 6$
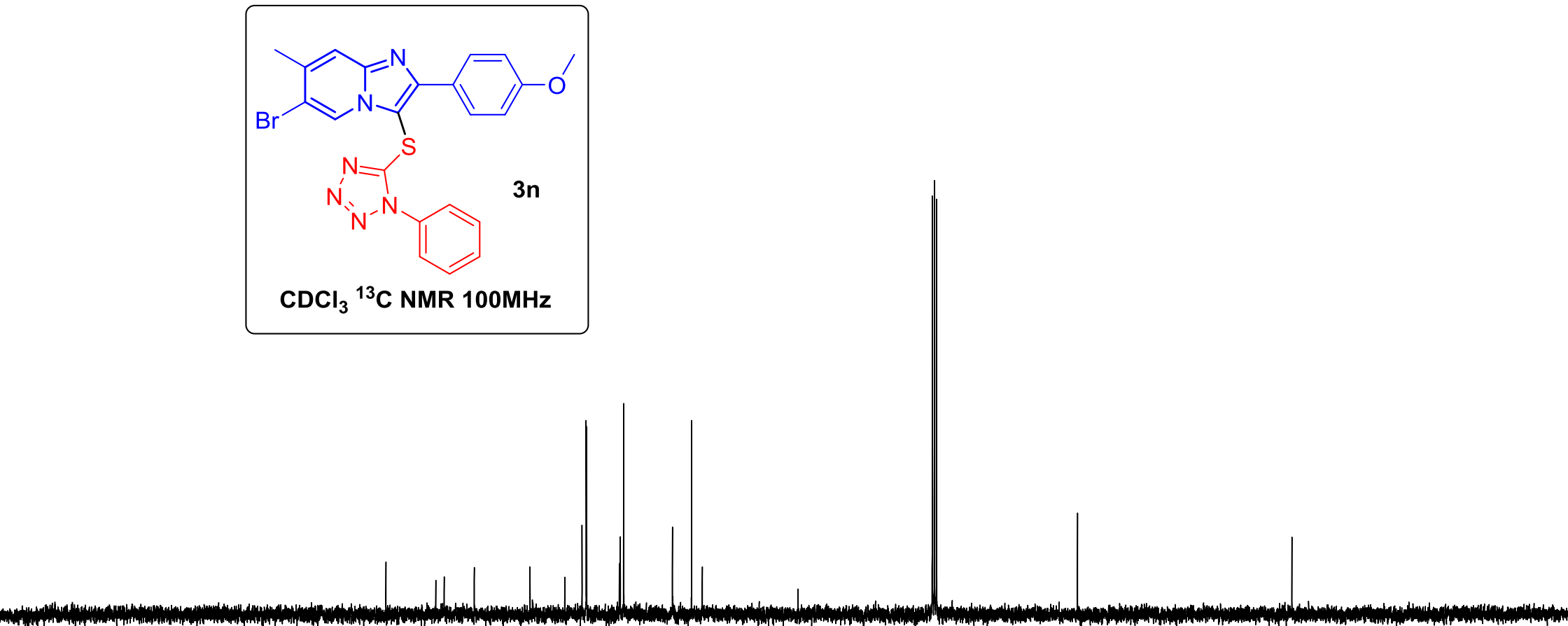

\begin{tabular}{rrrrrrrrrrrr}
\hline & 1 \\
\hline 200 & 180 & 160 & 140 & 120 & 100 & 80 & 60 & 40 & 20 & 0 & ppm
\end{tabular}




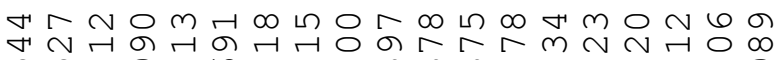

ज访

$\infty \infty \infty \sim \dot{r}$
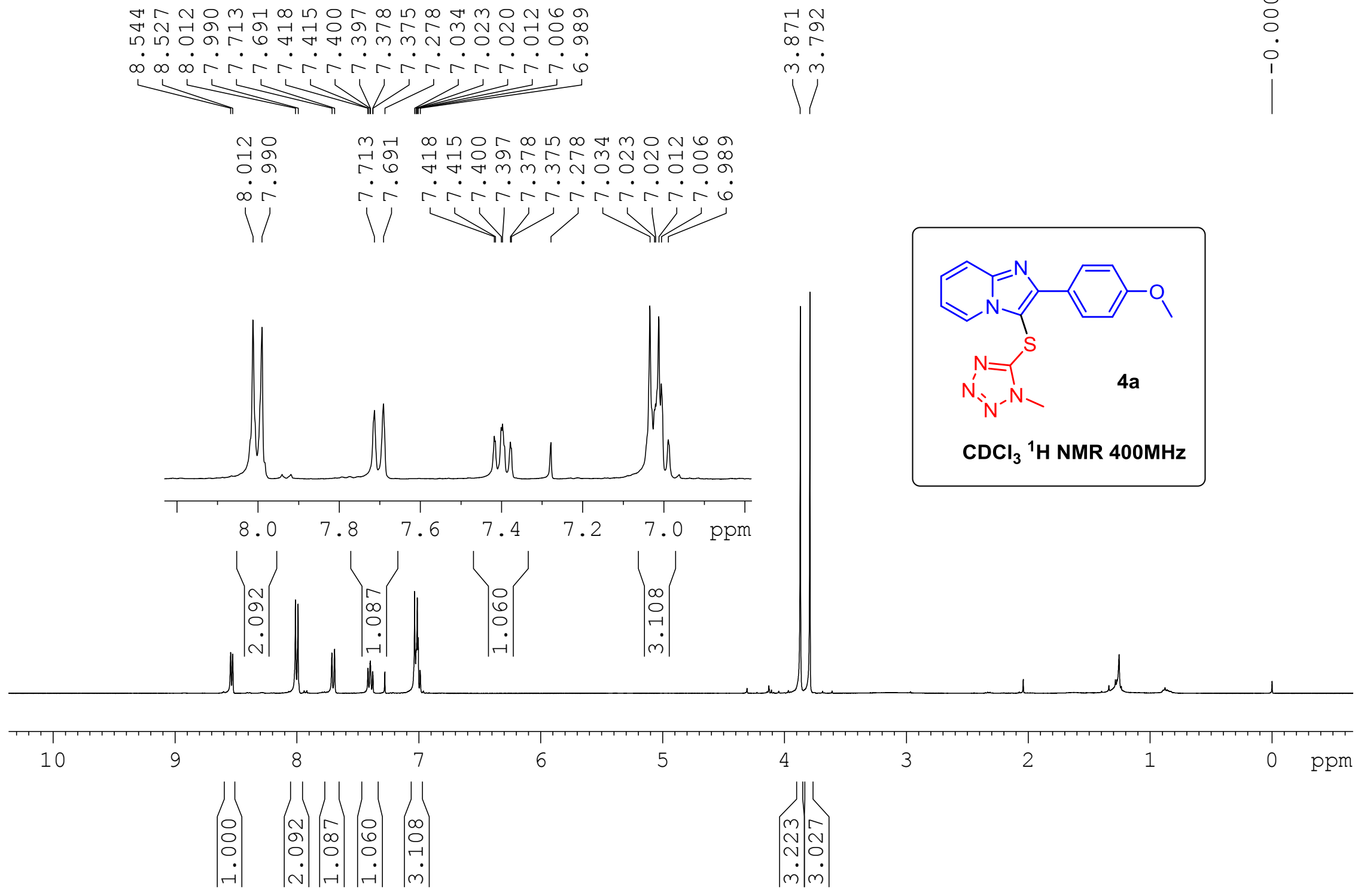

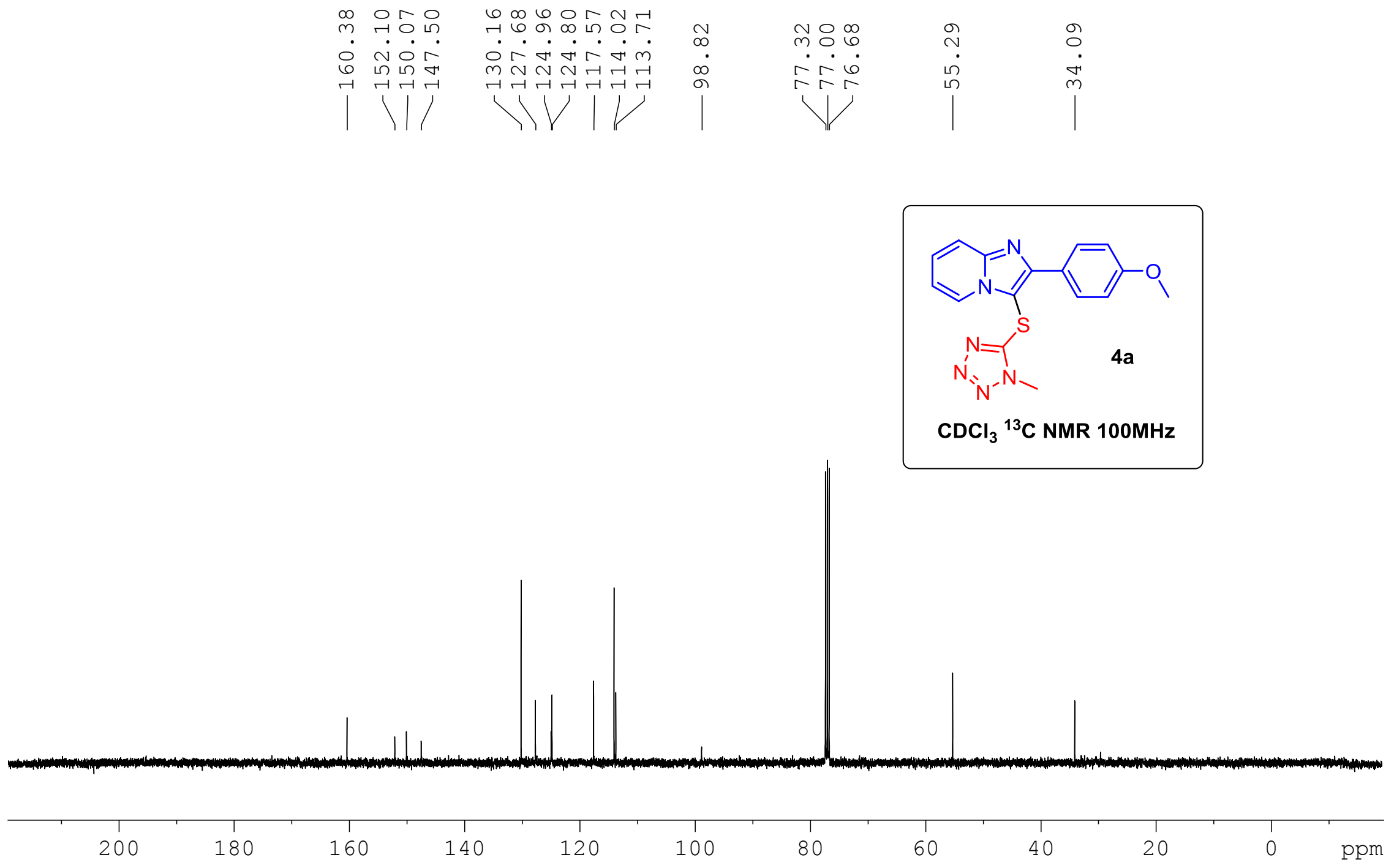


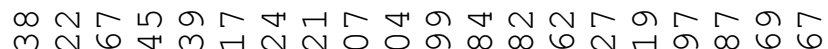
अ

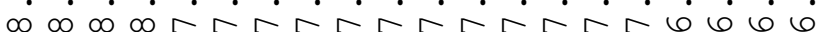
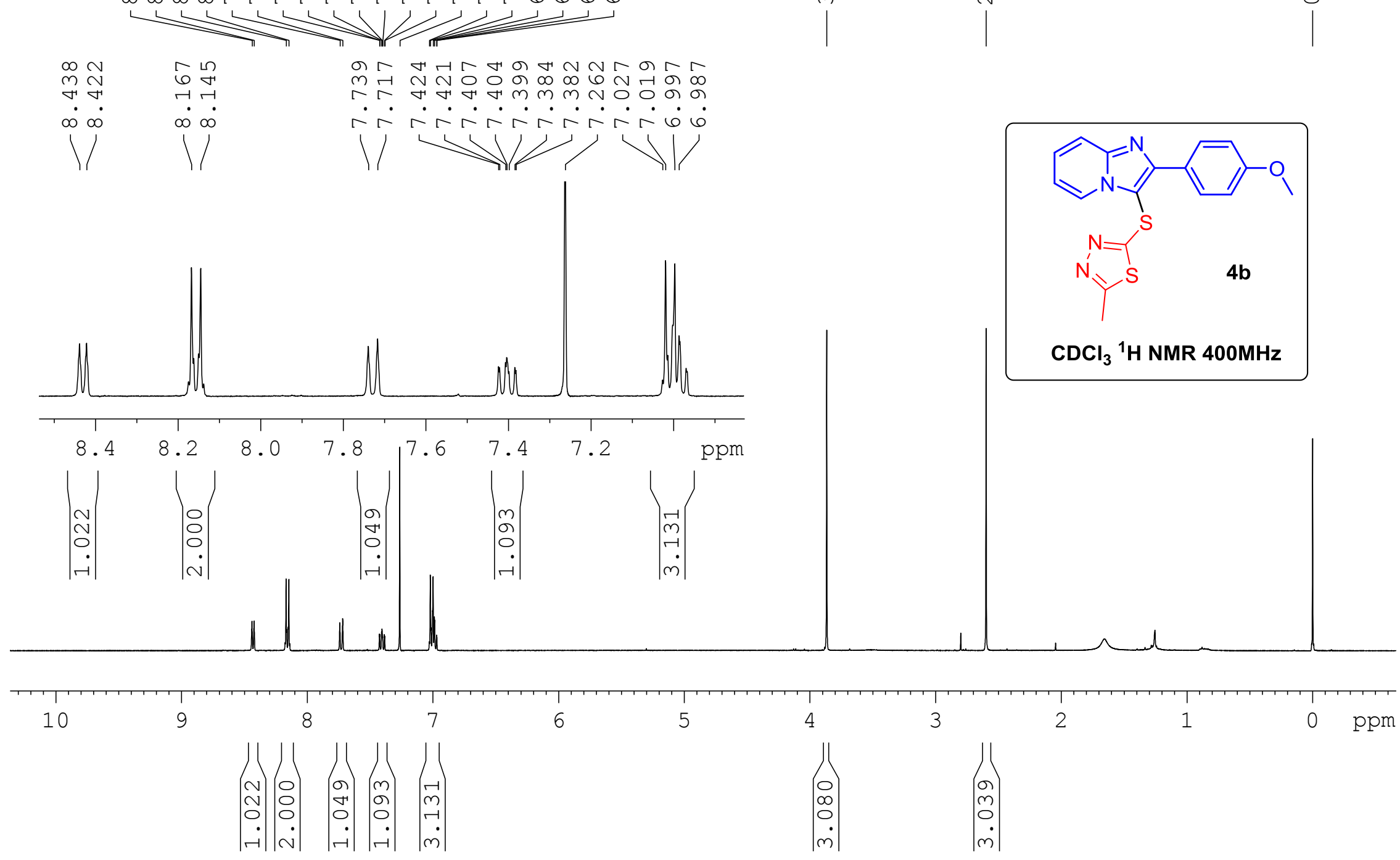


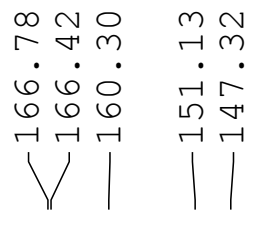

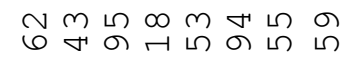

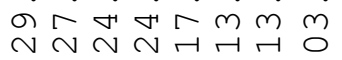

n̊

$\therefore \therefore 6$

$\therefore$

Y

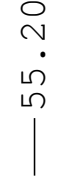

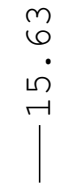
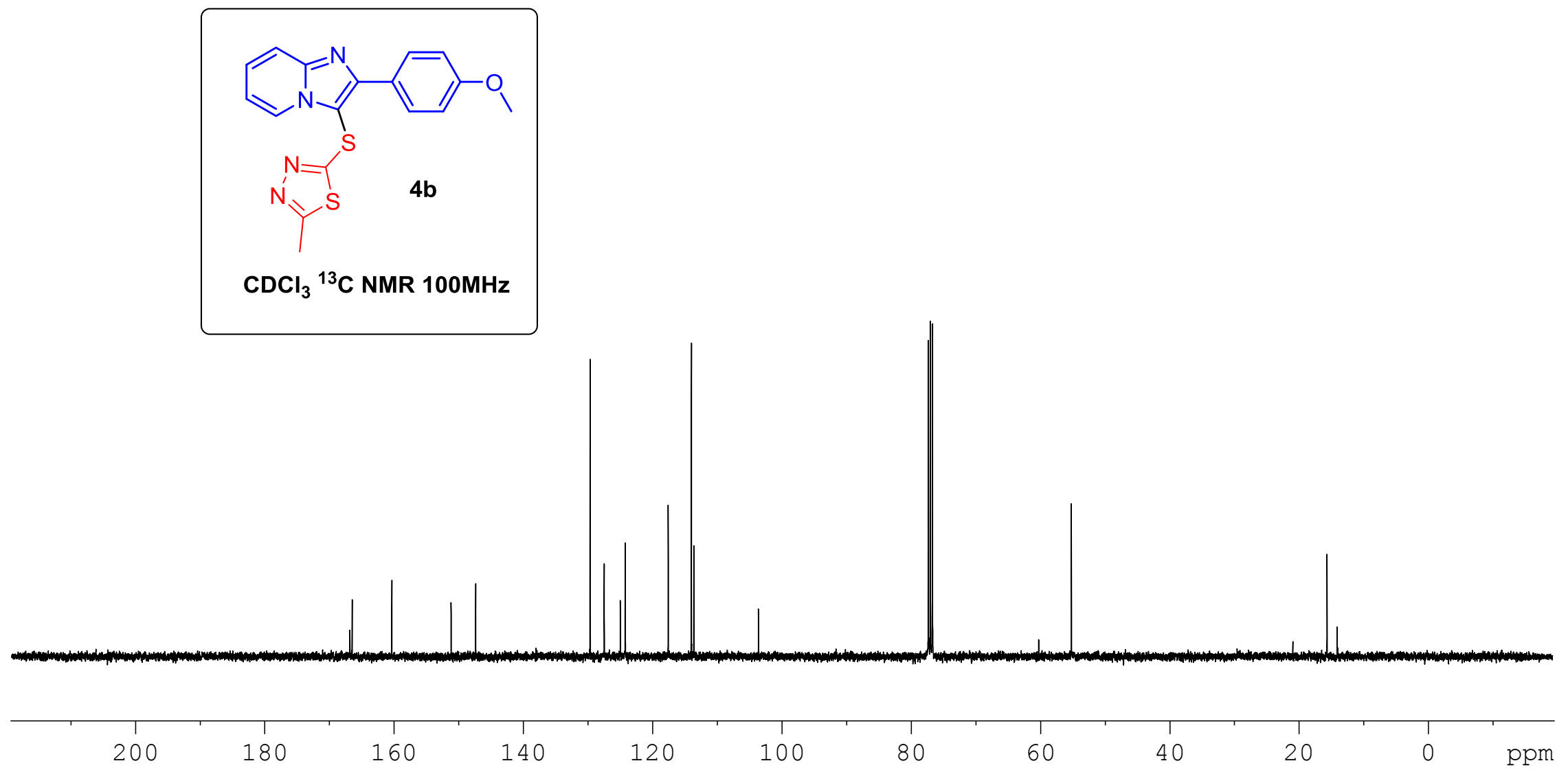
৫ Hrir

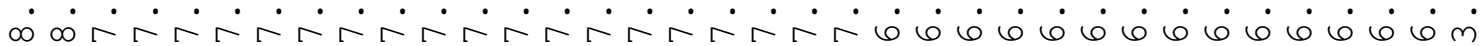
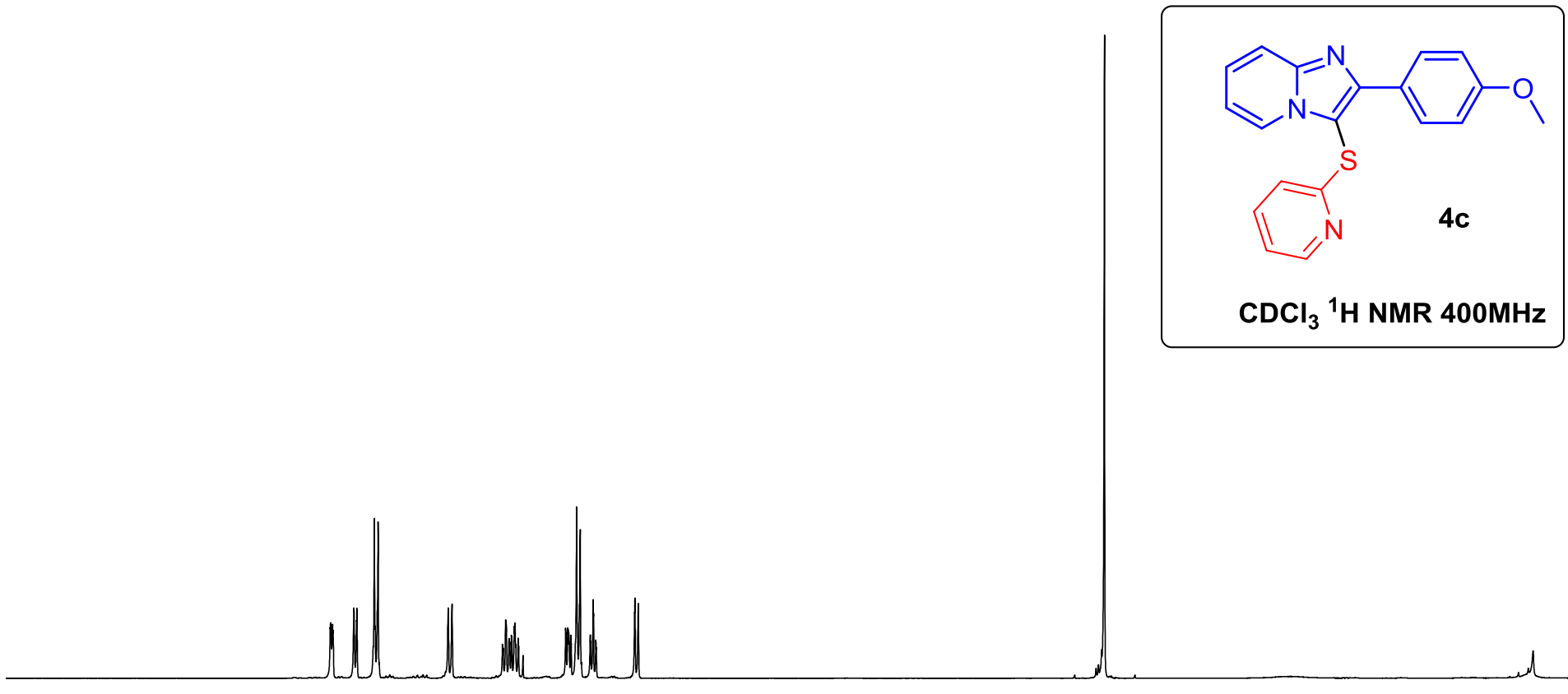

7

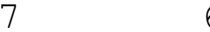

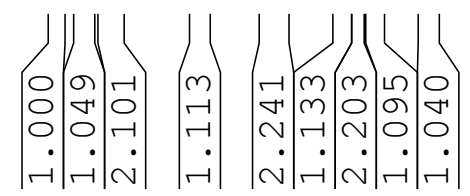

4

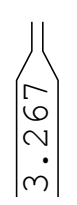

0 ppm 


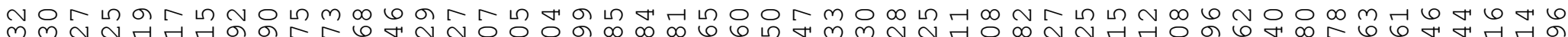

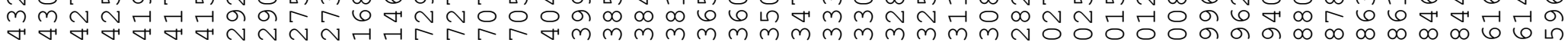

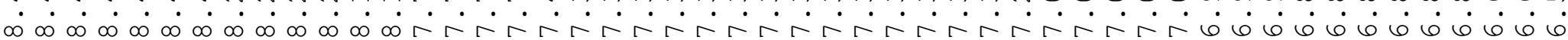
$\longrightarrow$
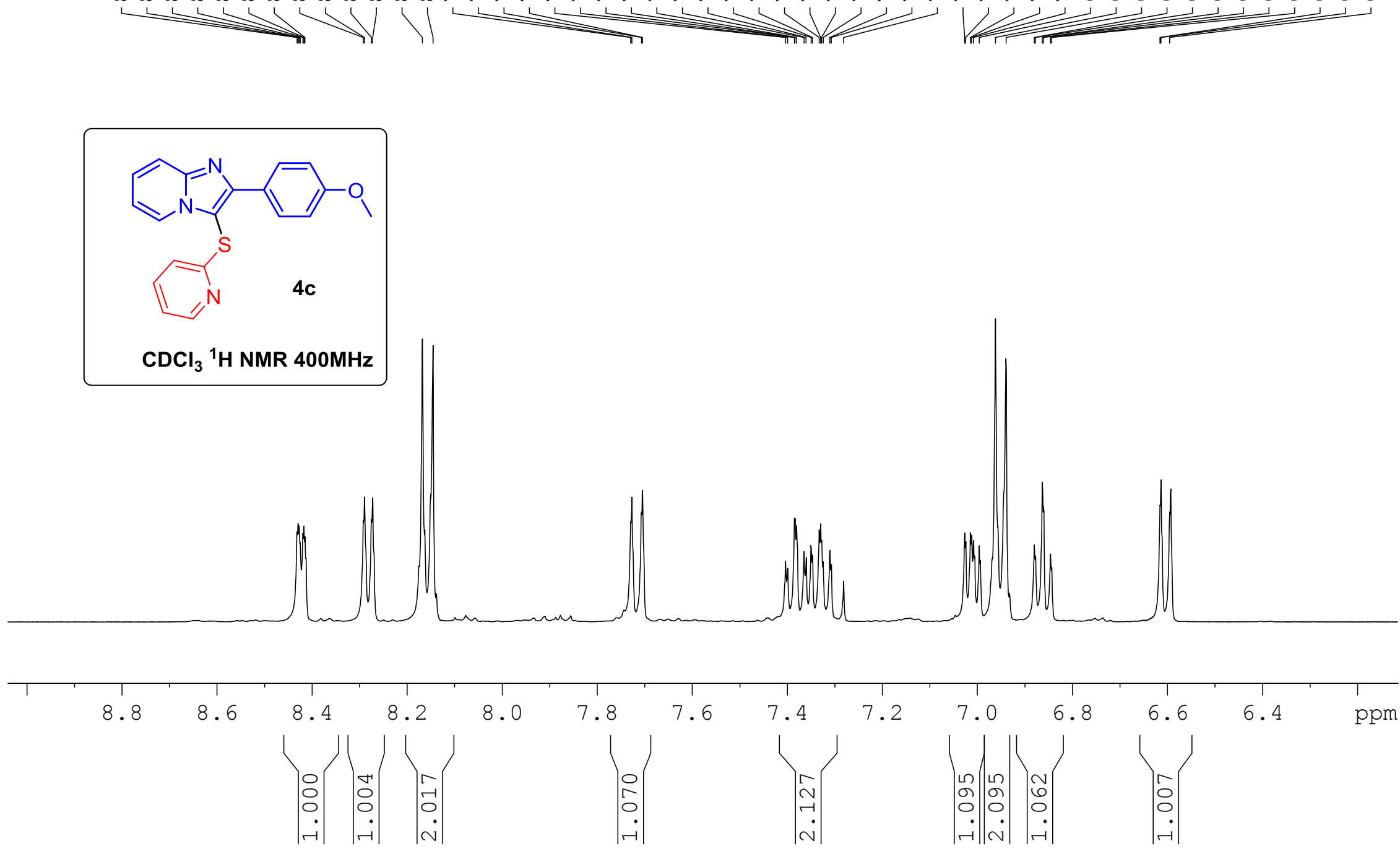


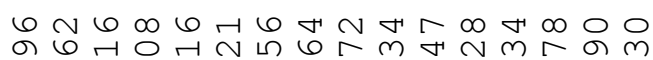
$\cdot \cdot \cdot \cdot \cdot \cdot \cdot \cdot \cdot \cdot \cdot \cdot \cdot$

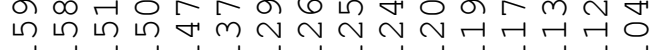

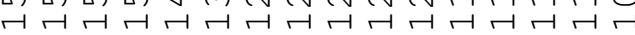

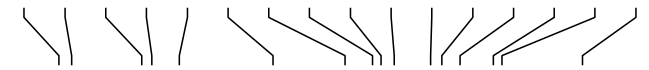

$$
\begin{aligned}
& \text { 긍요 } \\
& \text { 숭은 }
\end{aligned}
$$
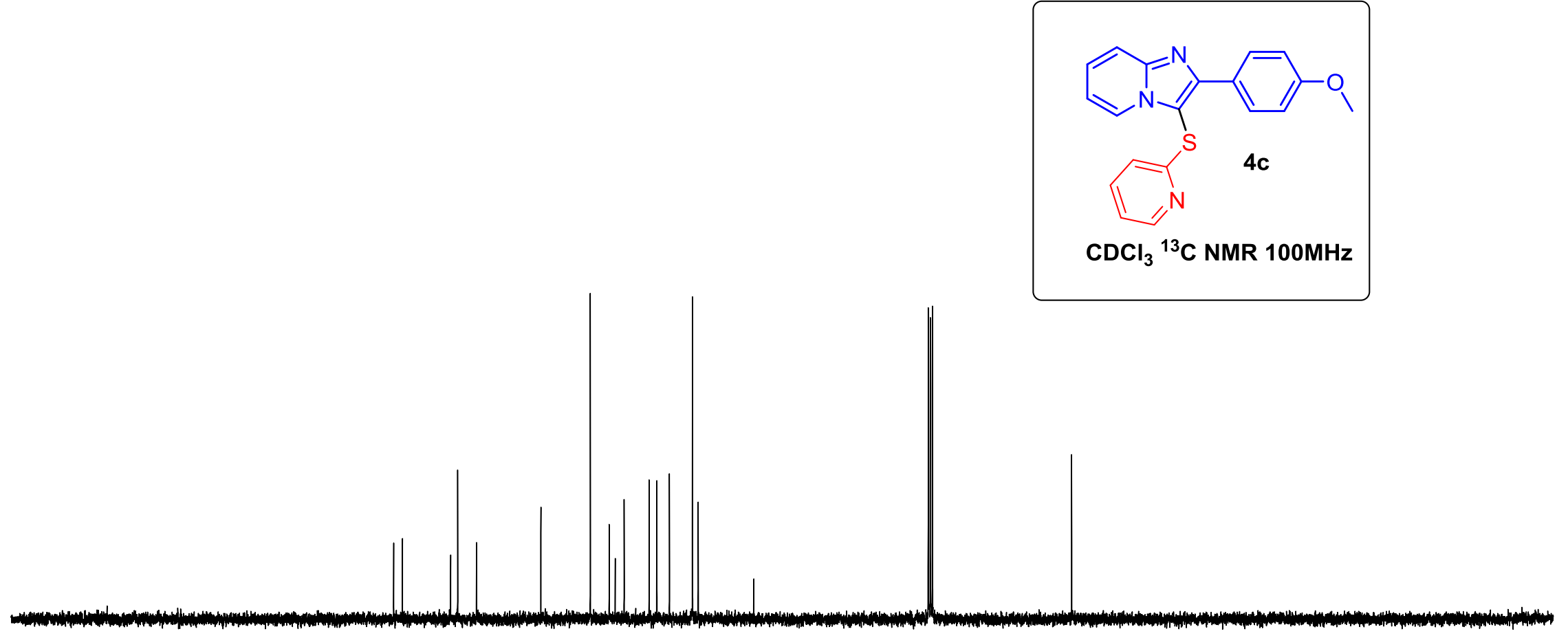

\begin{tabular}{rrrrrrrrrrrrr}
\hline & 1 \\
\hline 200 & 180 & 160 & 140 & 120 & 100 & 80 & 60 & 40 & 20 & 0 & ppm
\end{tabular}




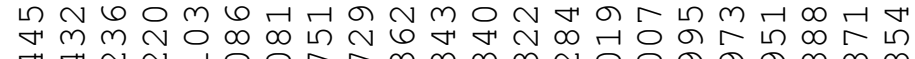

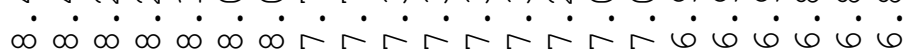
$\longrightarrow$

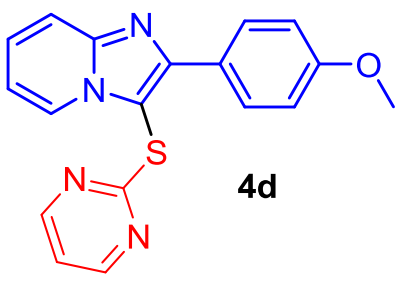

$\mathrm{CDCl}_{3}{ }^{1} \mathrm{H} \mathrm{NMR} 400 \mathrm{MHz}$

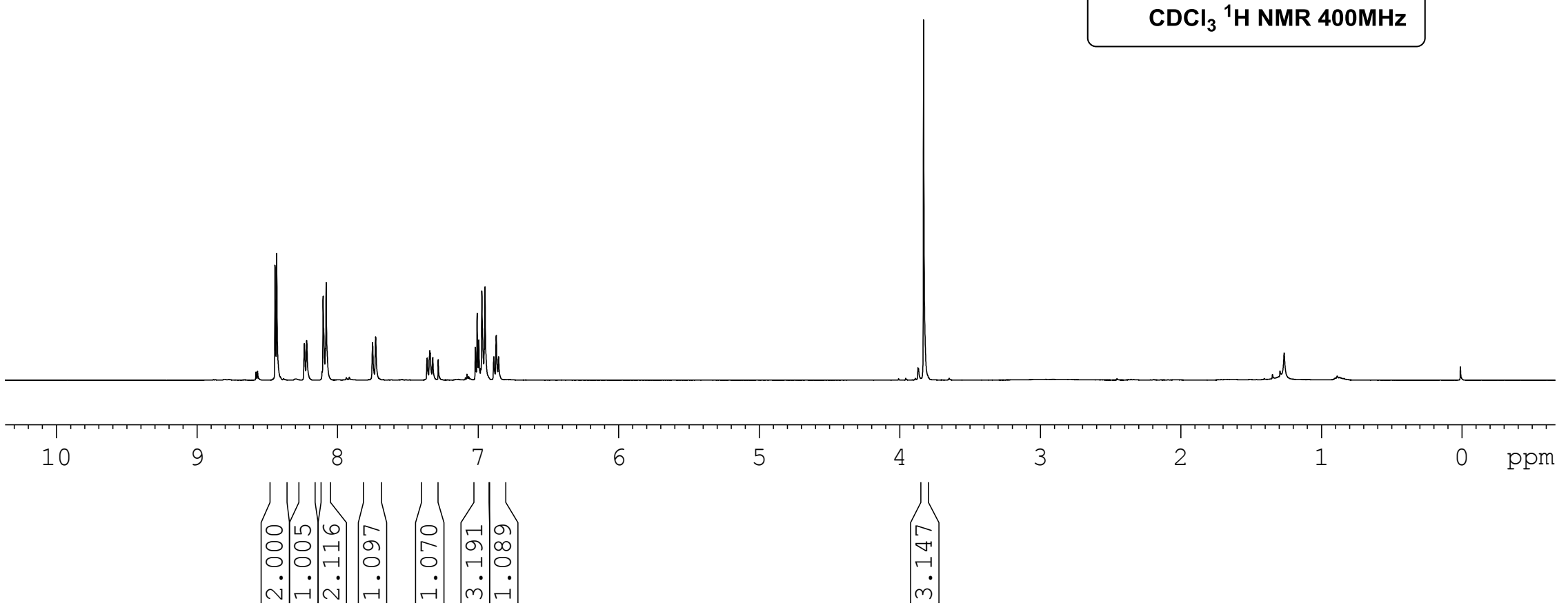



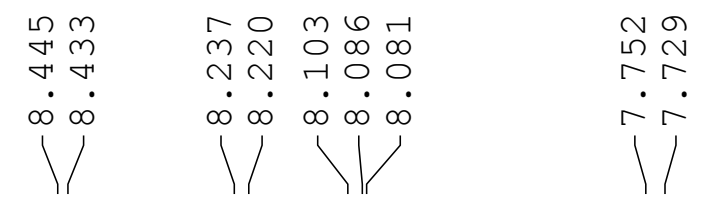

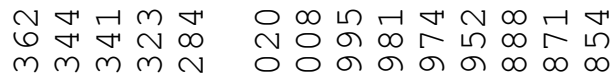
m.n. . . @ : ?

M/l

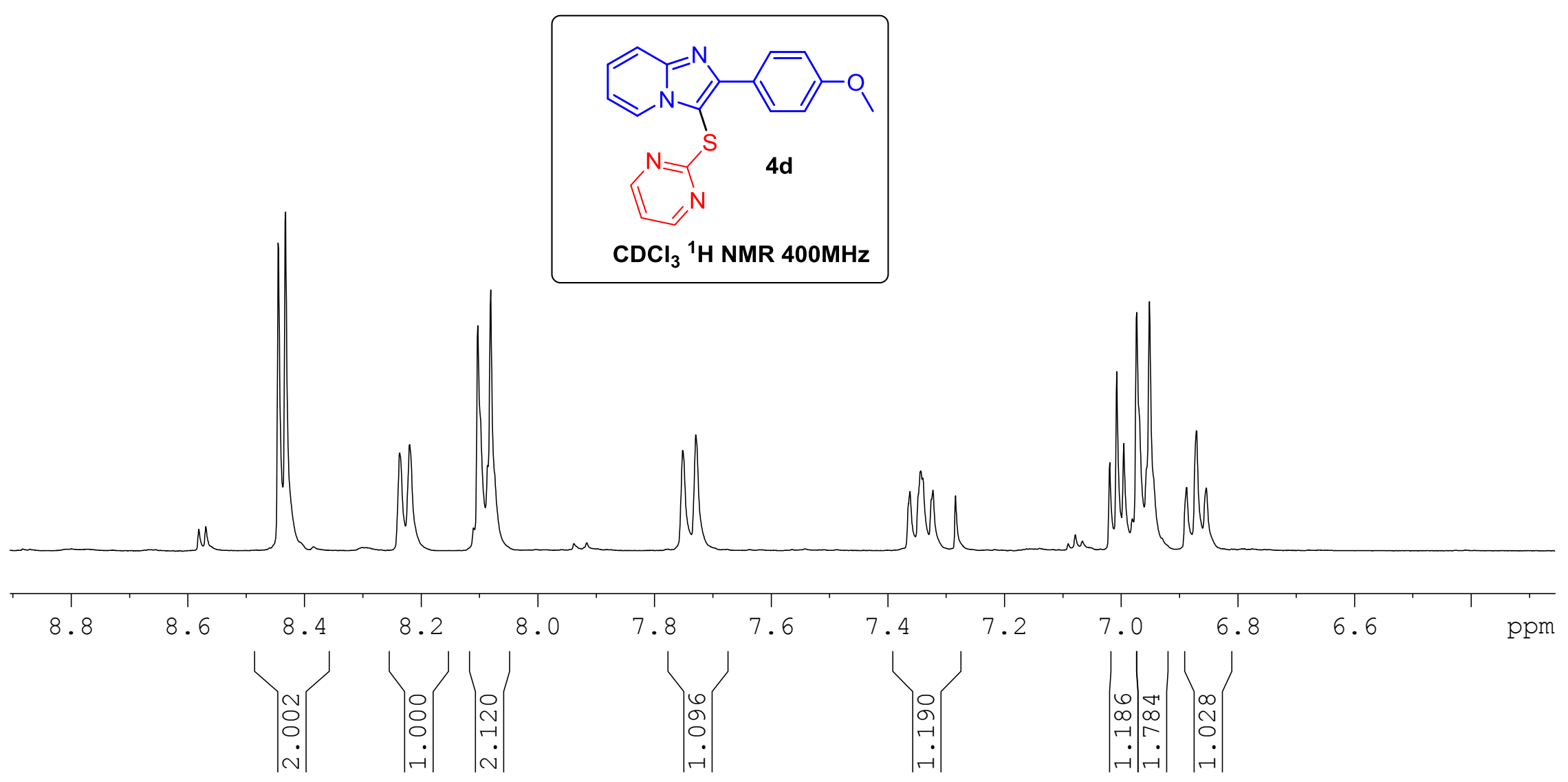



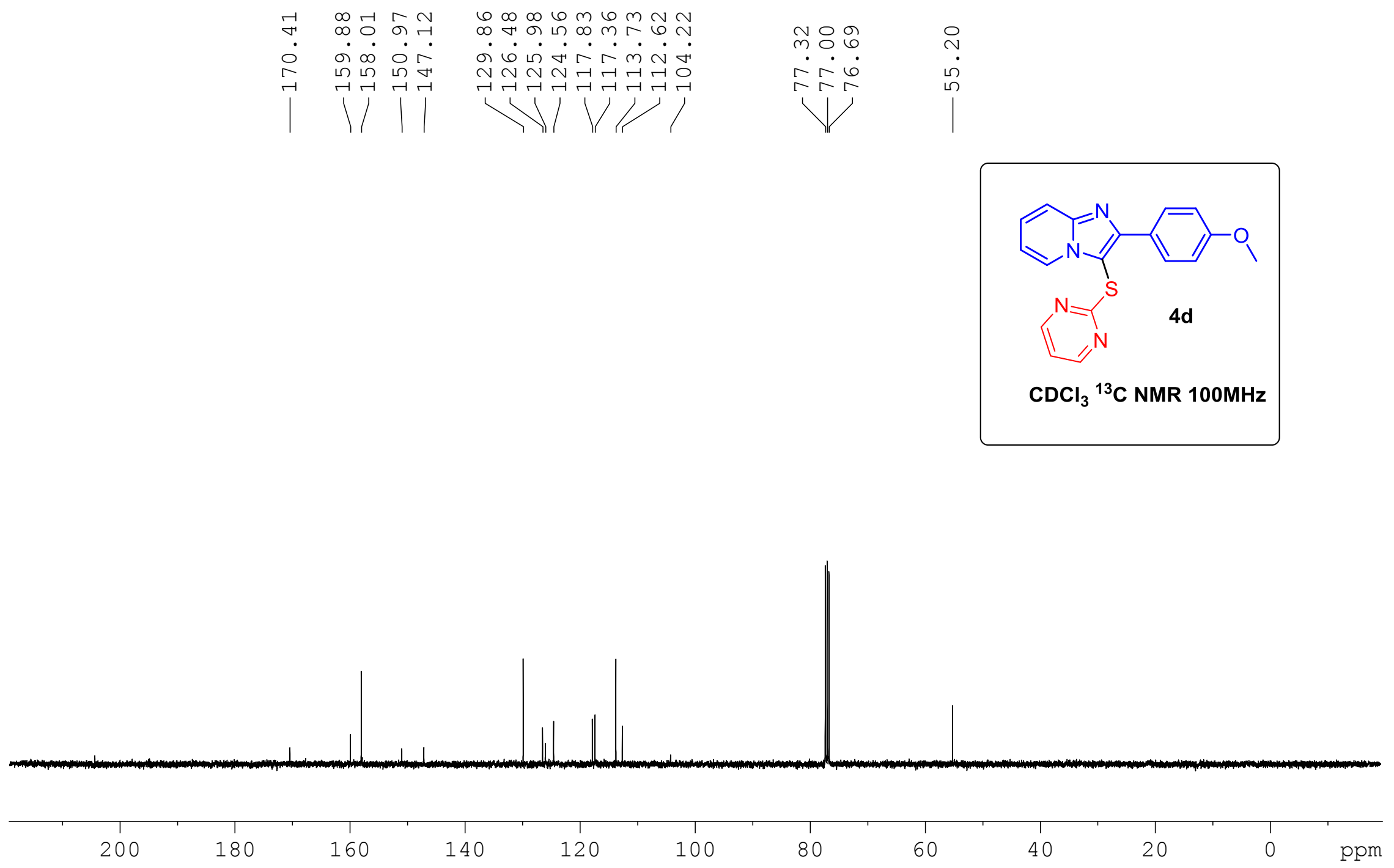


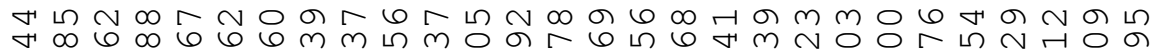

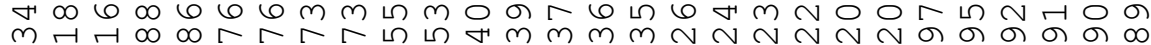
o o o 0

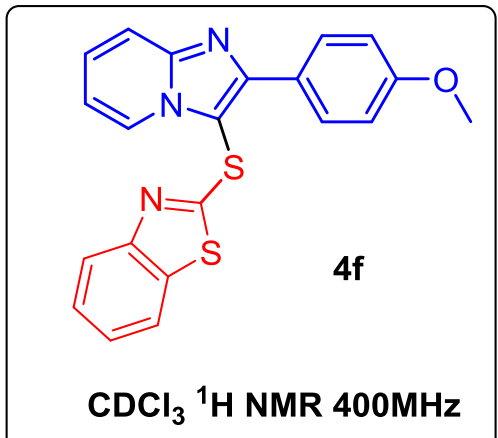

\section{$\mathrm{CDCl}_{3}{ }^{1} \mathrm{H}$ NMR $400 \mathrm{MHz}$}

1) mul

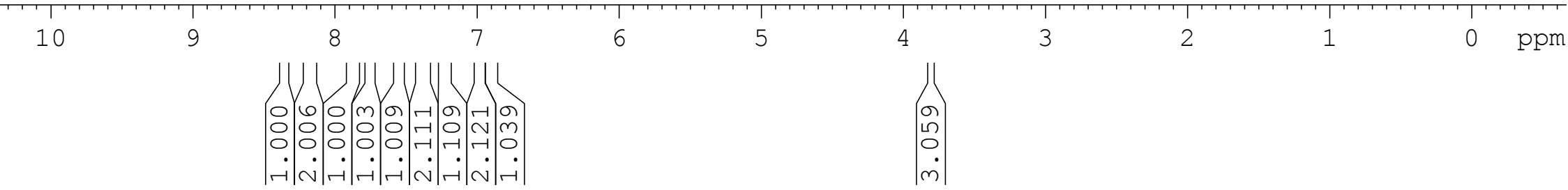




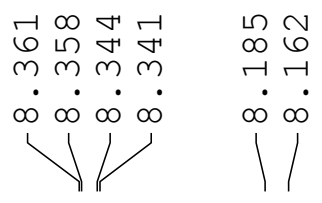

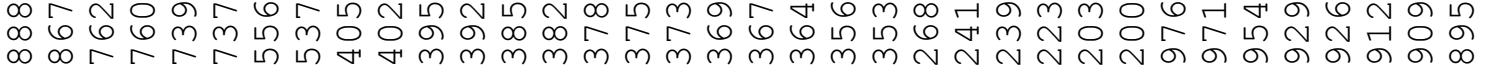
o
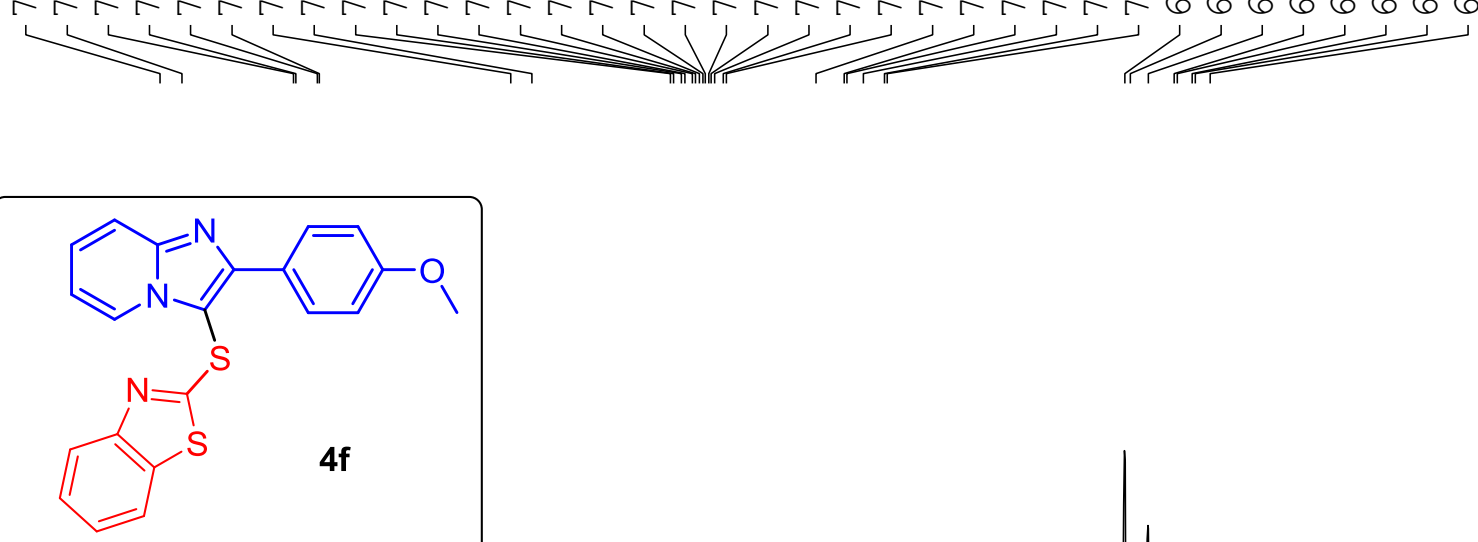

$\mathrm{CDCl}_{3}{ }^{1} \mathrm{H}$ NMR 400MHz
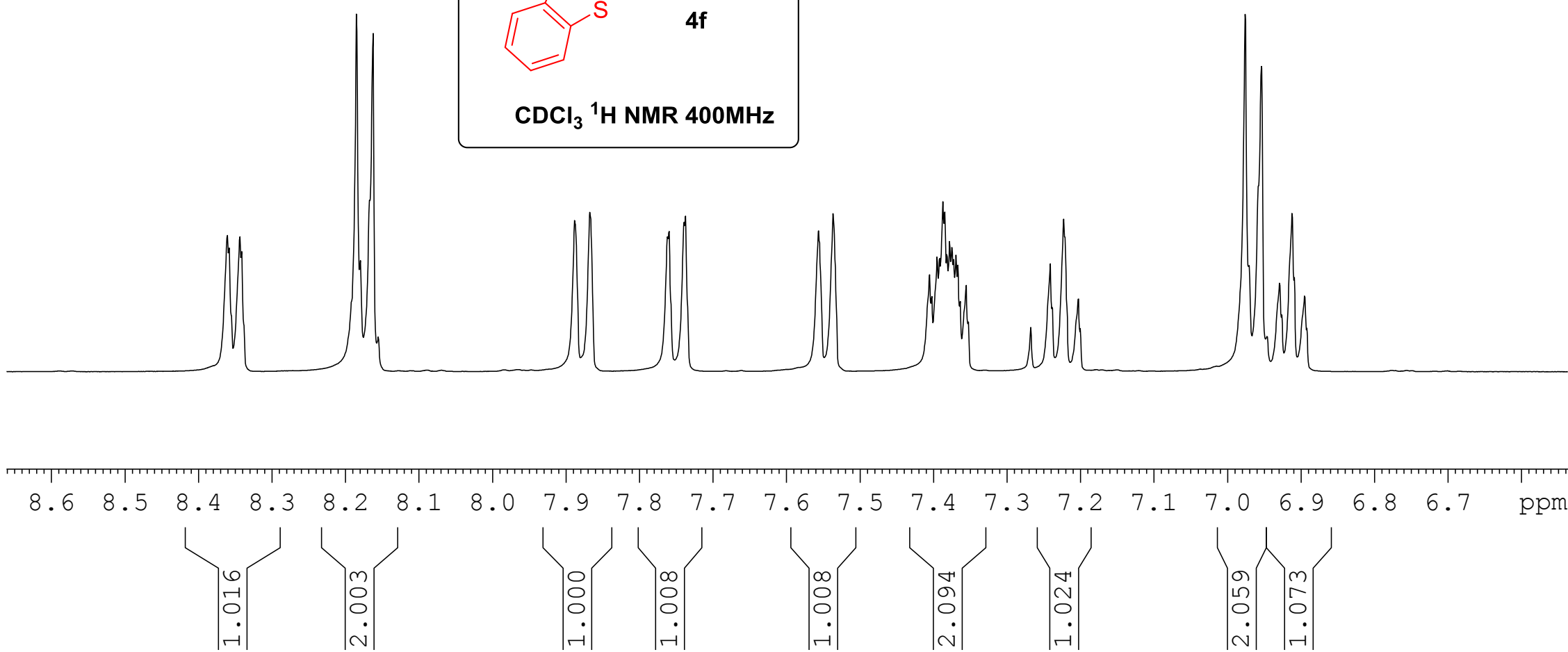


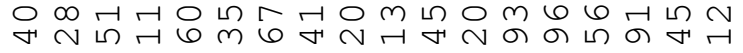
$\infty$ iñ

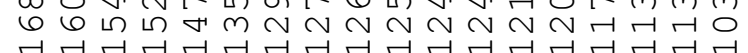
$1<1<1$

ñ॰

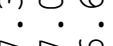

รัก

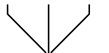

$\stackrel{r}{\stackrel{r}{r}}$

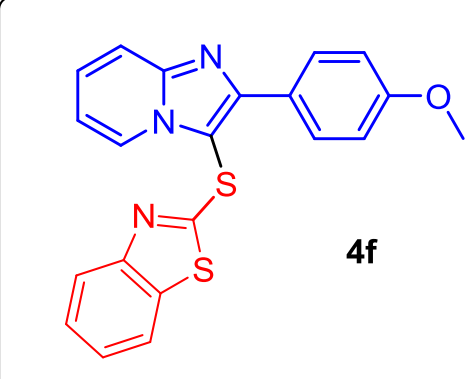

$\mathrm{CDCl}_{3}{ }^{13} \mathrm{C}$ NMR $100 \mathrm{MHz}$

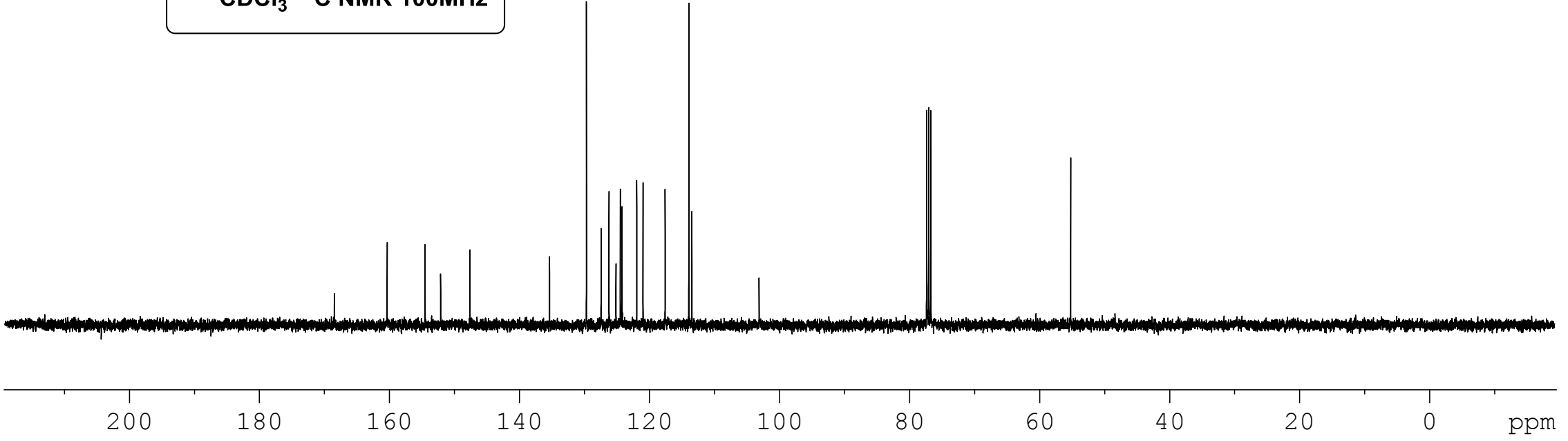


ம

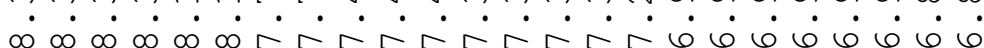

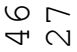

$\infty \infty$

$\dot{m} \dot{m}$
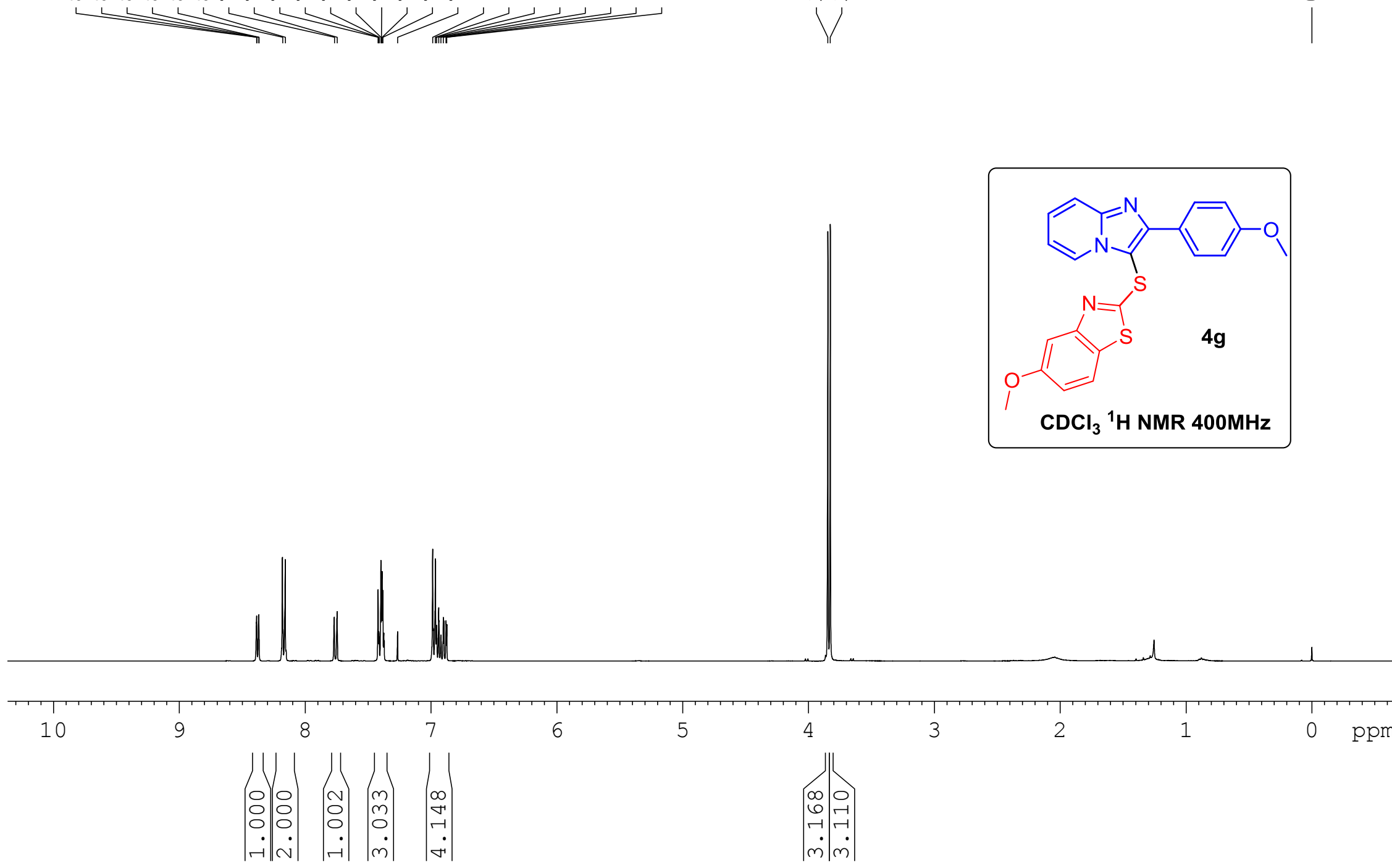


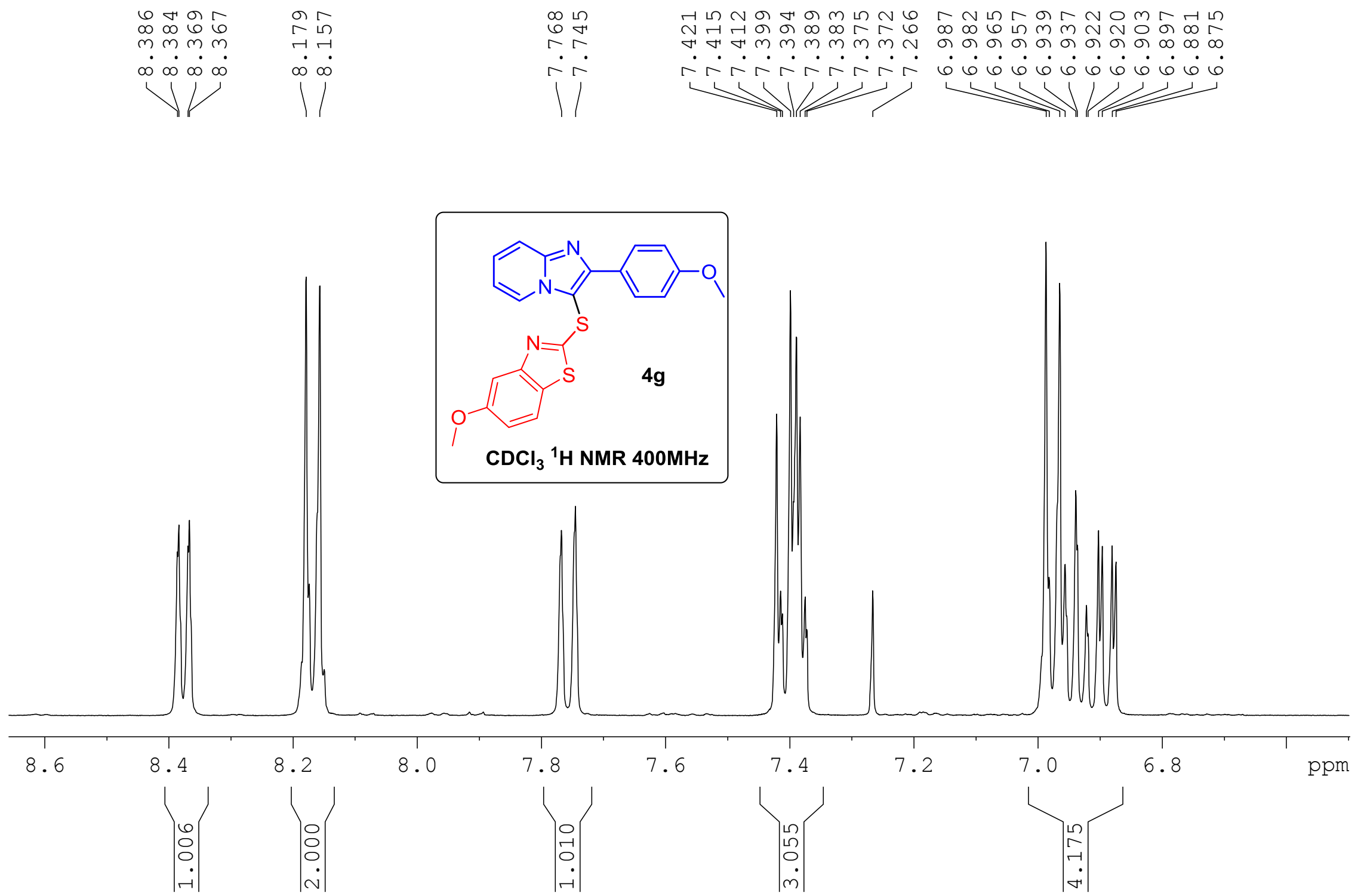


மூ

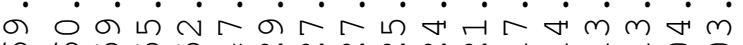

6
0

1111111

mo

$\therefore \dot{0}$

$ヘ \stackrel{1}{\sim}$

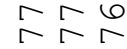

م.

ถัก

V

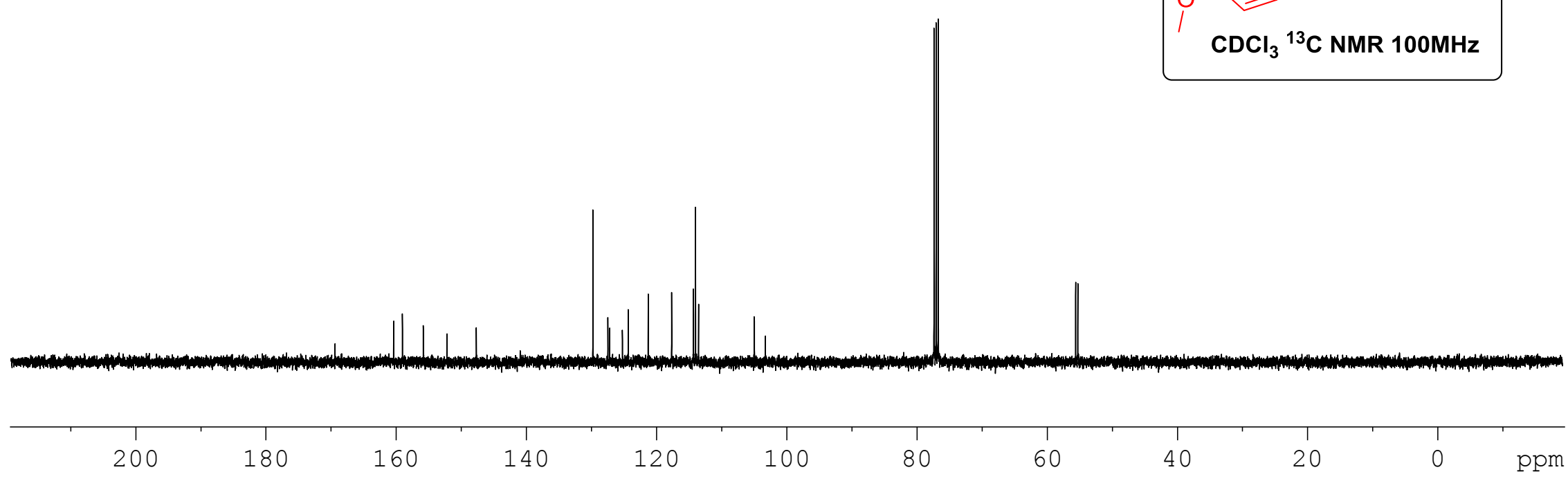




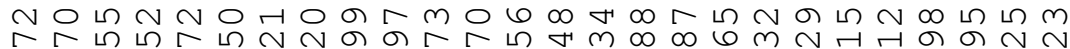

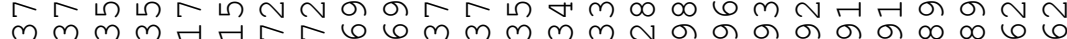

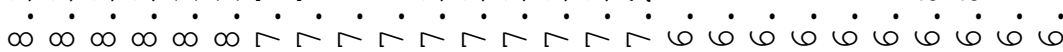

L

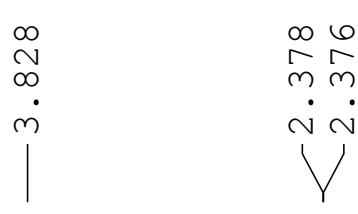

$\circ$
$\circ$
$\circ$
0
1

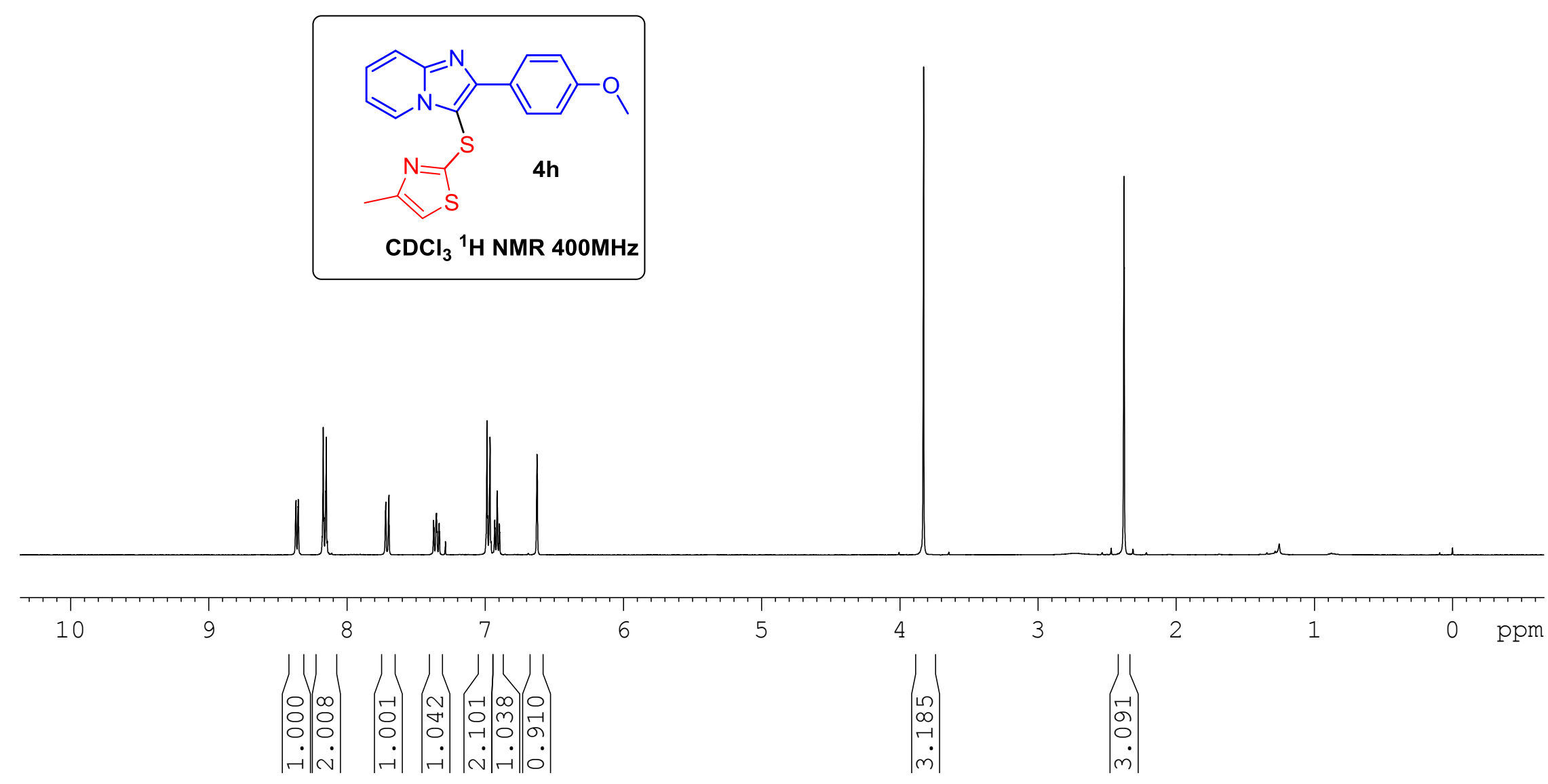

V

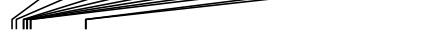

$\begin{array}{ll}0 & \circ \\ \stackrel{0}{m} & 0 \\ \dot{j} & 0 \\ \dot{j} & 1\end{array}$



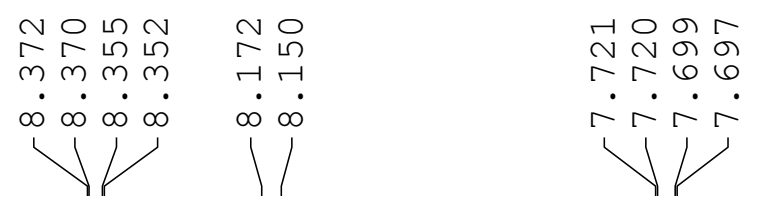

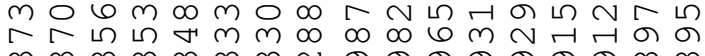

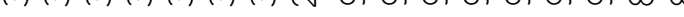

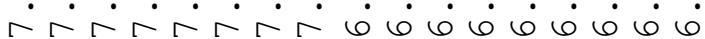

$\longrightarrow$
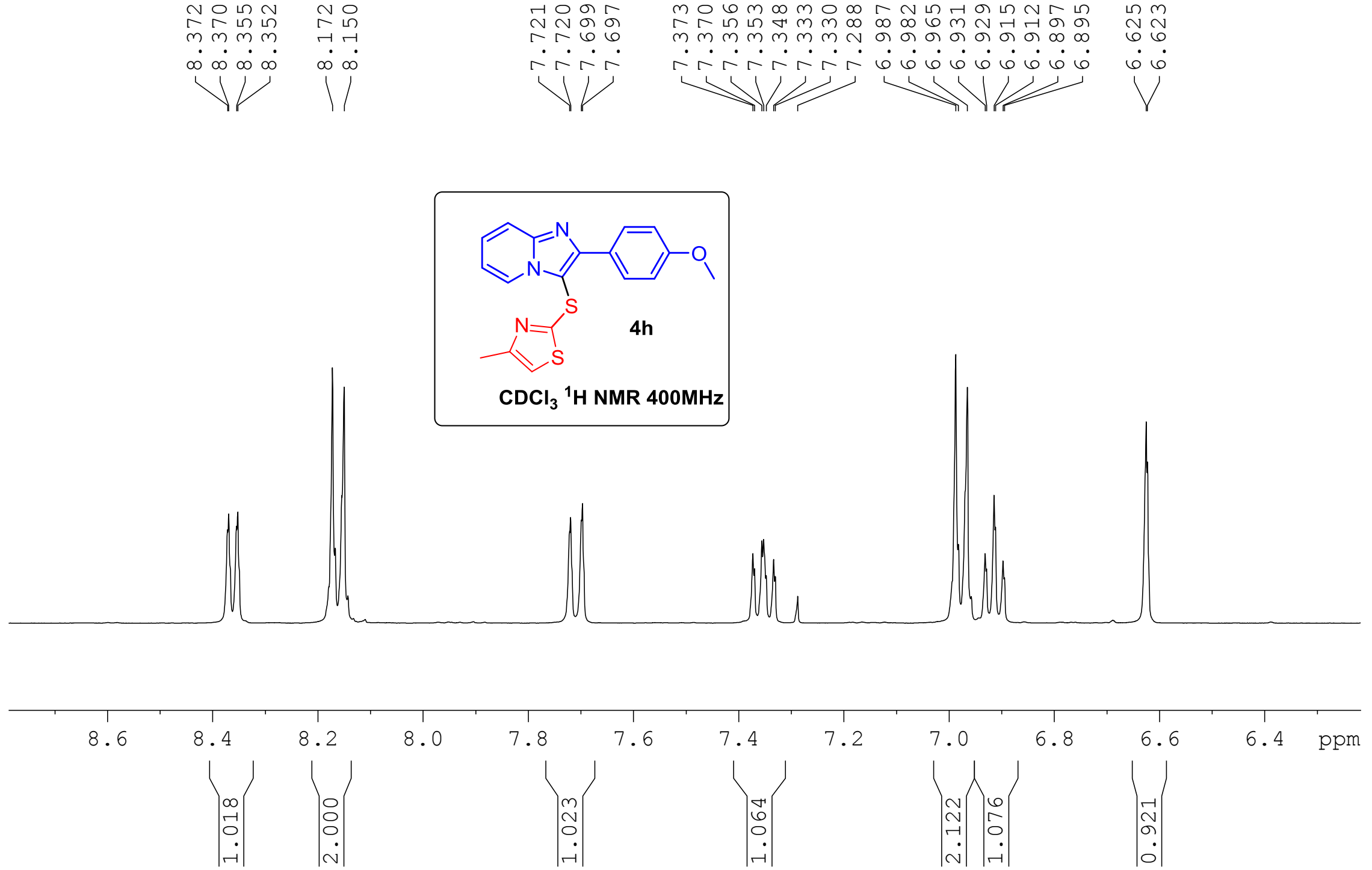
$\stackrel{6}{\circ} \stackrel{6}{m} \stackrel{m}{m}$

aिं मiि

68 ㄷำ

111

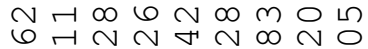

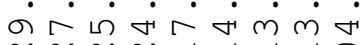

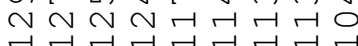

)।

$\mathrm{CDCl}_{3}{ }^{13} \mathrm{C}$ NMR $100 \mathrm{MHz}$

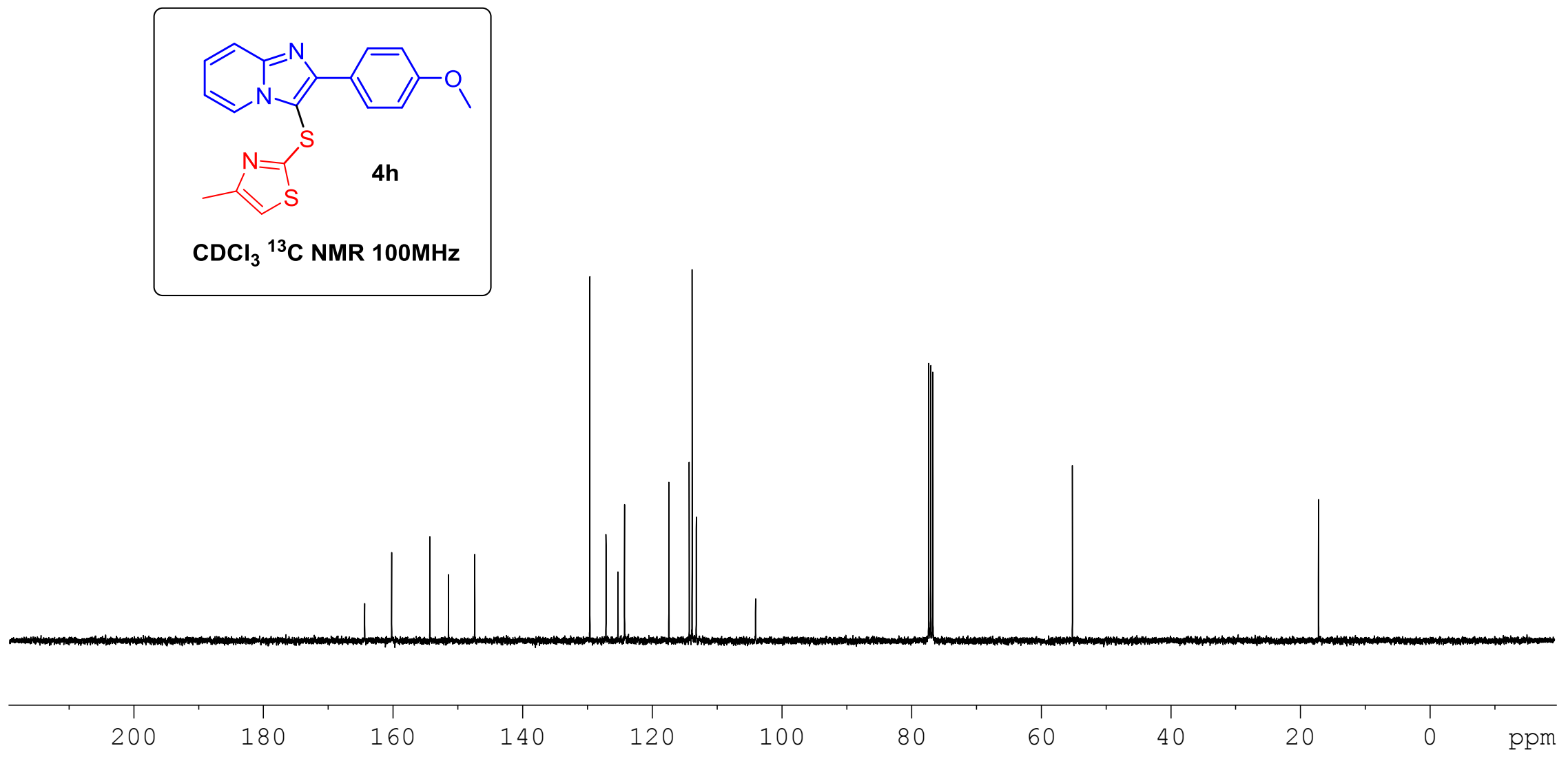

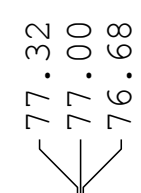

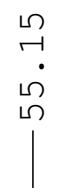

\[ \]
$\stackrel{-}{r}$
$\stackrel{-}{r}$ 


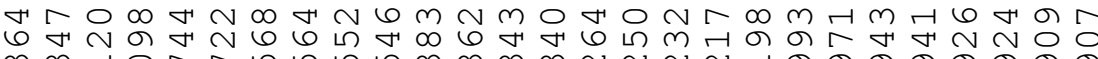
$m m+\infty r$ o 0000

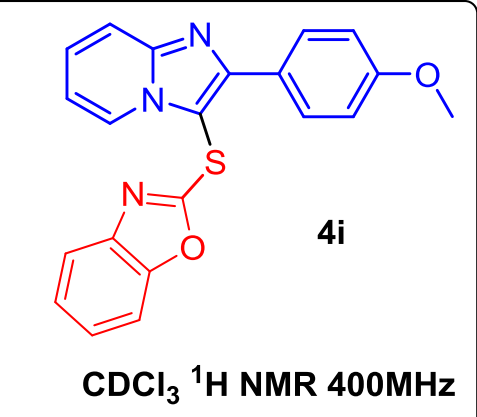

$\mathrm{CDCl}_{3}{ }^{1} \mathrm{H}$ NMR $400 \mathrm{MHz}$
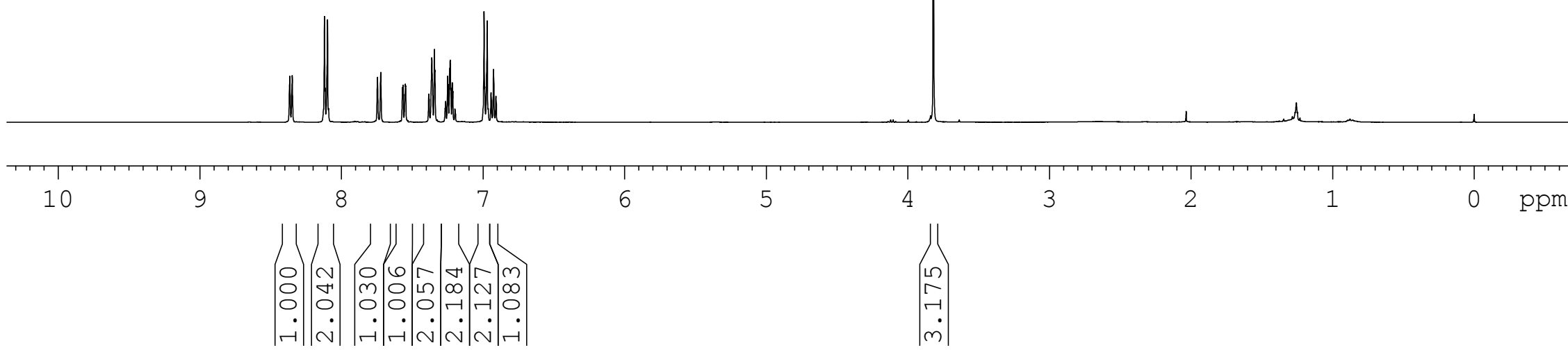

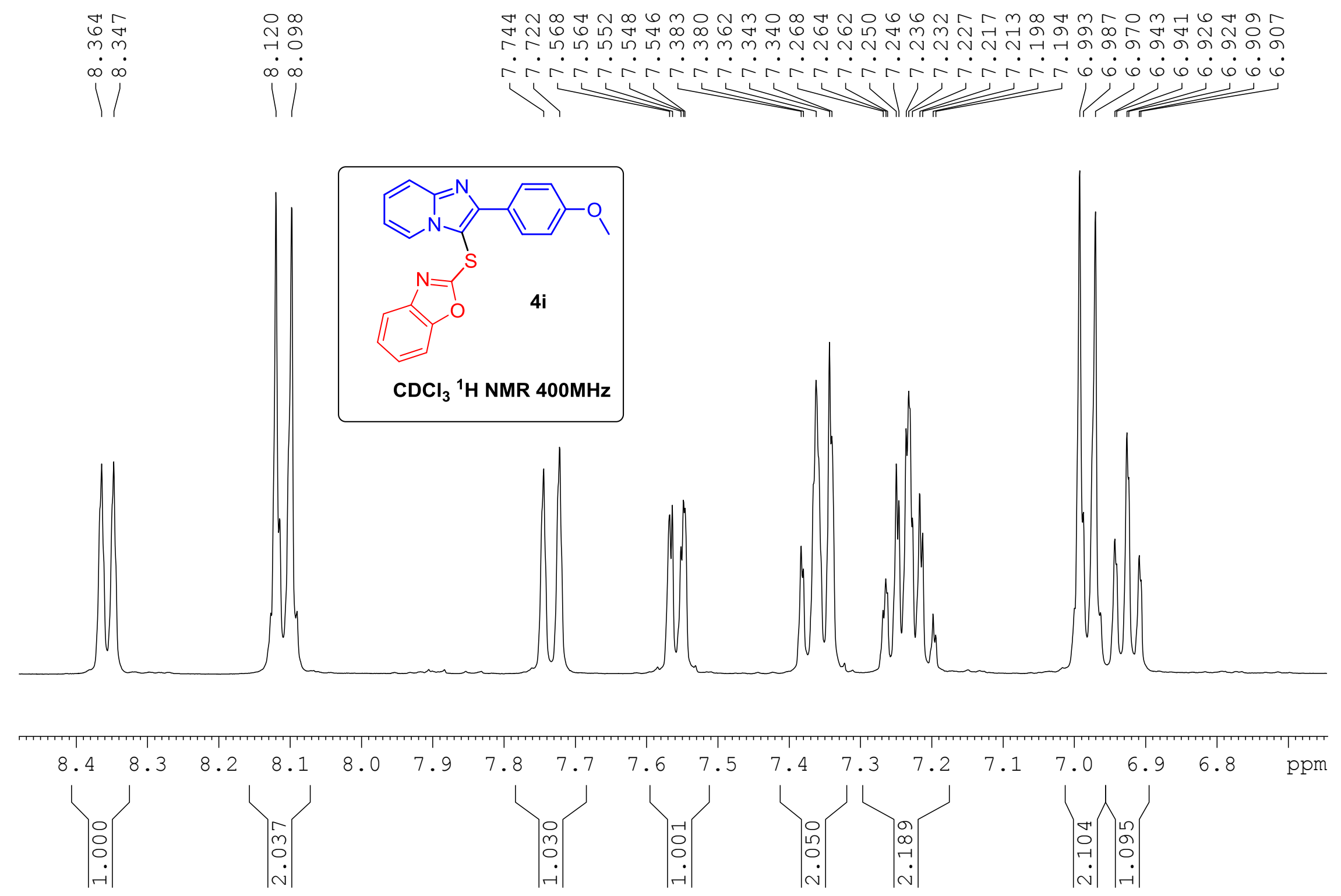


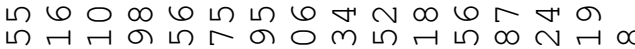

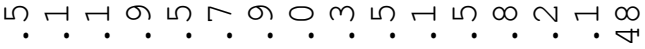

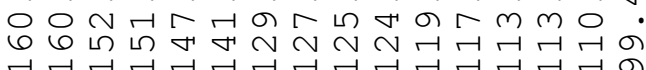

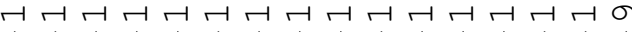

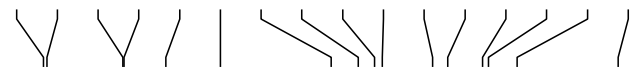

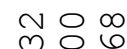

m.

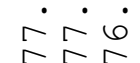

11

$\stackrel{n}{r}$

$\stackrel{\llcorner}{n}$

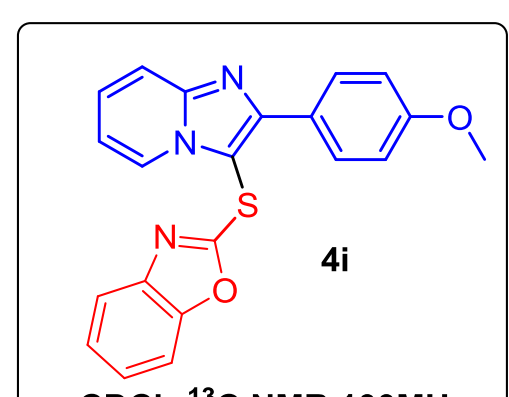

$\mathrm{CDCl}_{3}{ }^{13} \mathrm{C}$ NMR $100 \mathrm{MHz}$

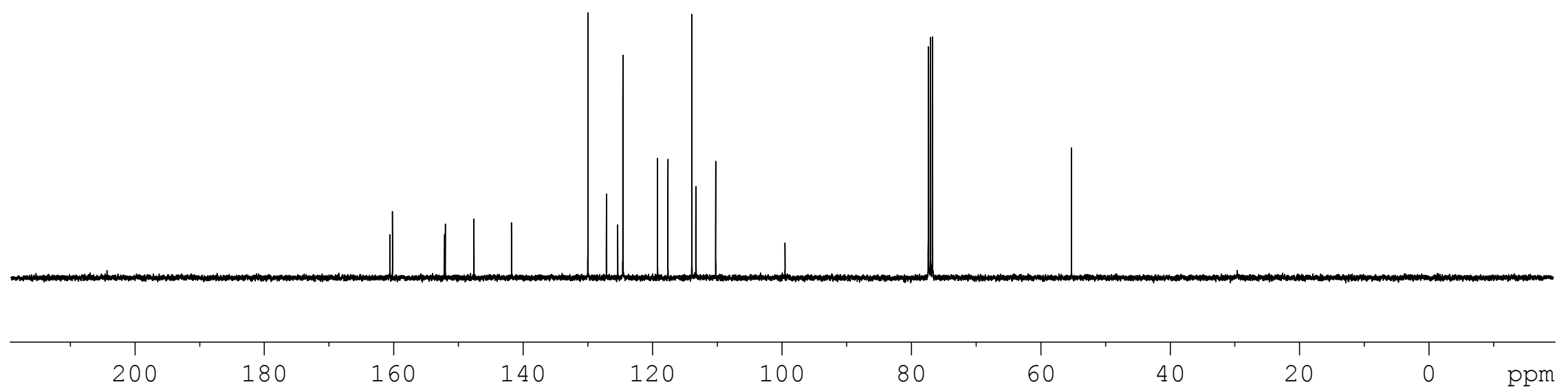




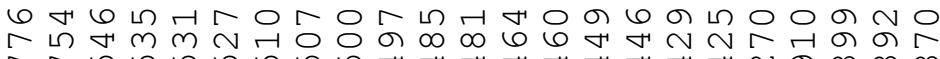

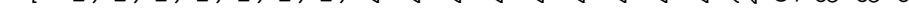

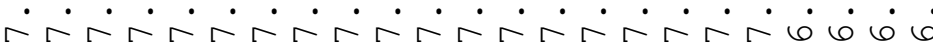

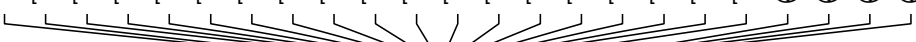

4 4๐の

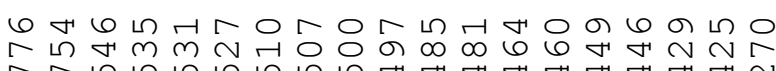

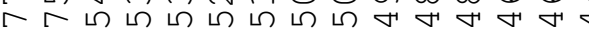

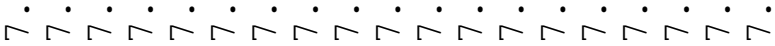

$\longrightarrow$

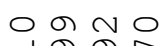
नुळ $\dot{\omega} \dot{0} \dot{0}$ $1 /$
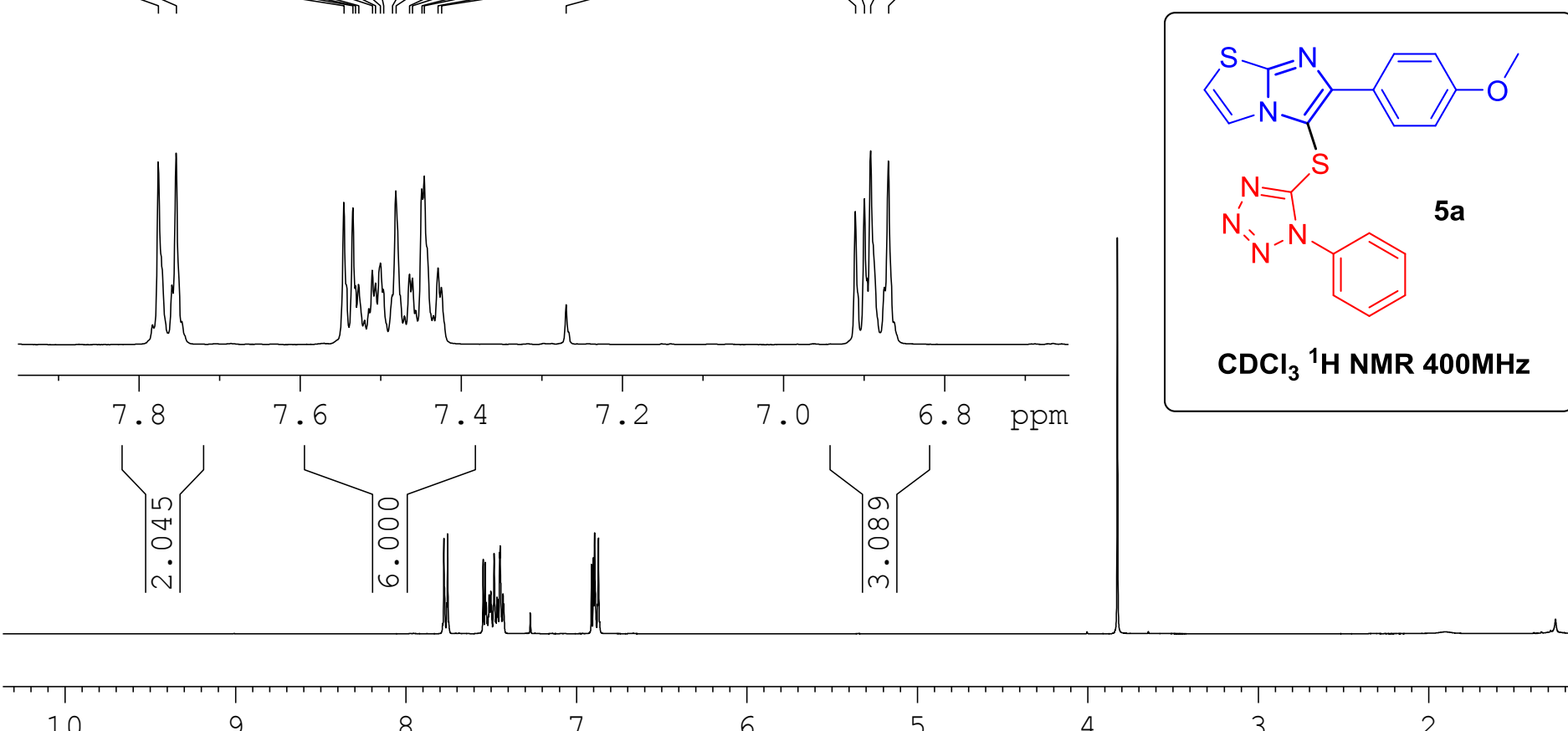

9

8

$\left|\begin{array}{c}n \\ \sim \\ + \\ 0 \\ ن\end{array}\right|\left|\begin{array}{l}\mid \\ 0 \\ 0 \\ 0 \\ 0\end{array}\right|$

$\begin{aligned} & 7 \\ & 0 \\ & 0 \\ & 0 \\ & 0 \\ & m\end{aligned} \mid$

5

4

3

2

1

0 ppm 


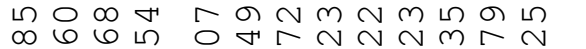

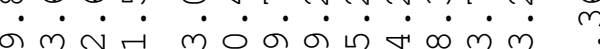

เn

l/ $1 / 1 / 1$
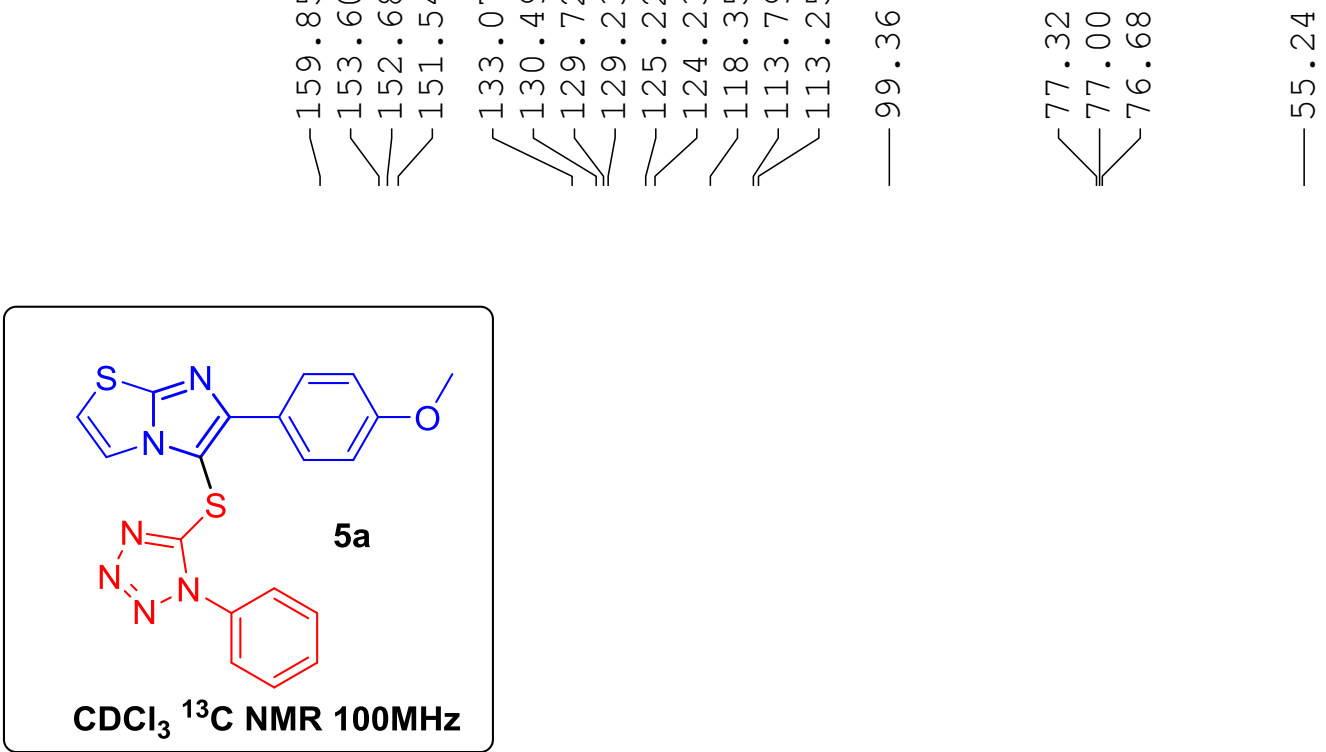

${ }^{3} \mathrm{C}$ NMR $100 \mathrm{MHz}$

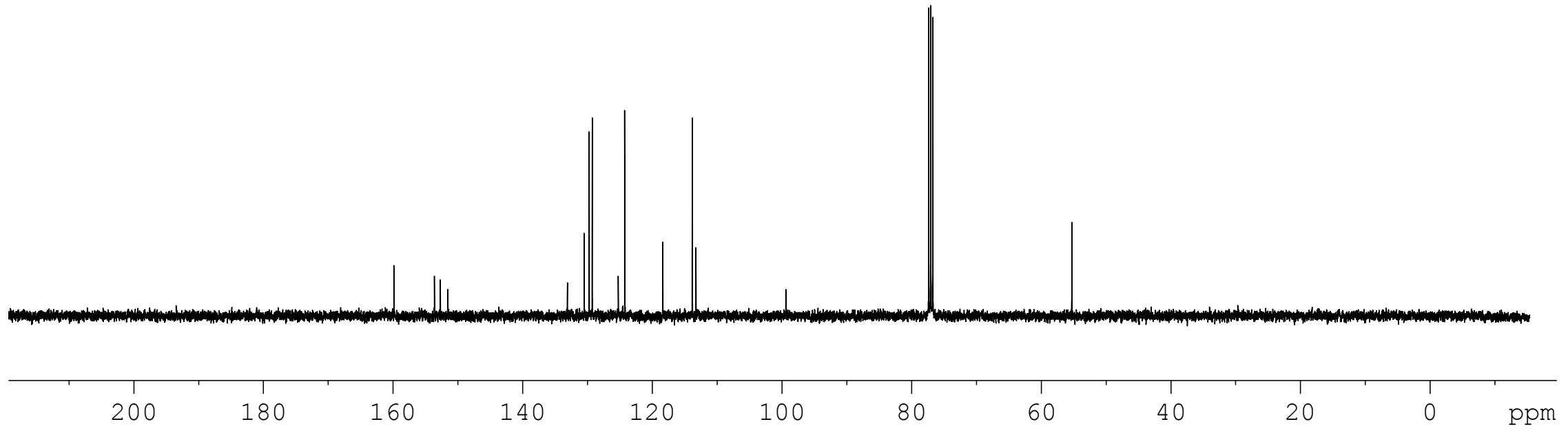




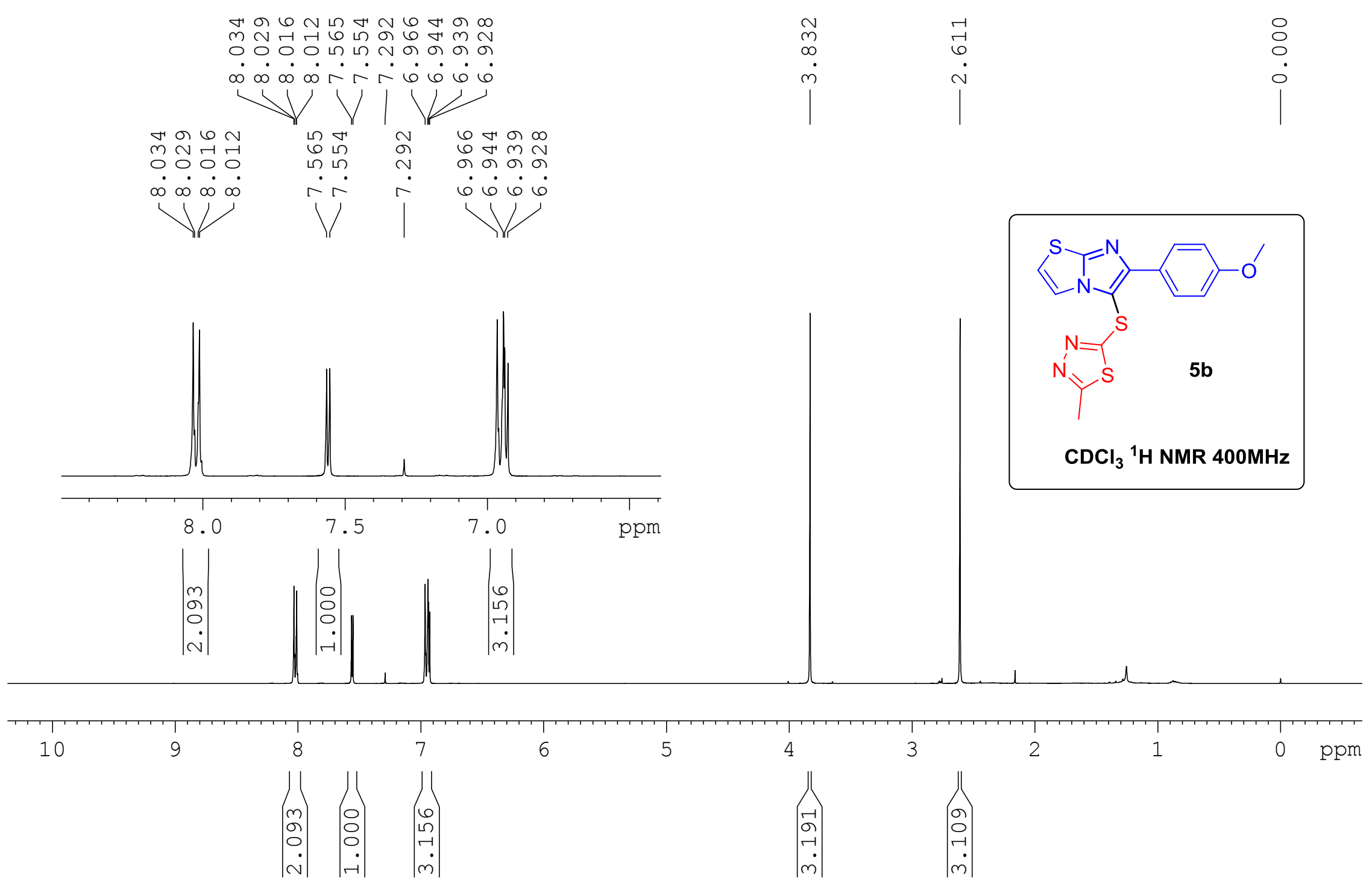




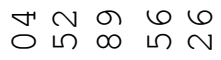

$\therefore \dot{0} \sim \dot{0}$

ถู)

नमतनम

VV

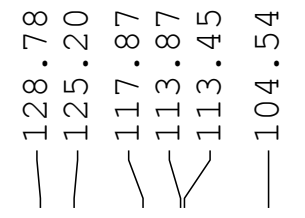

m $\circ 0$

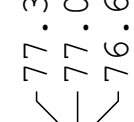

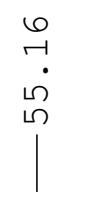

|
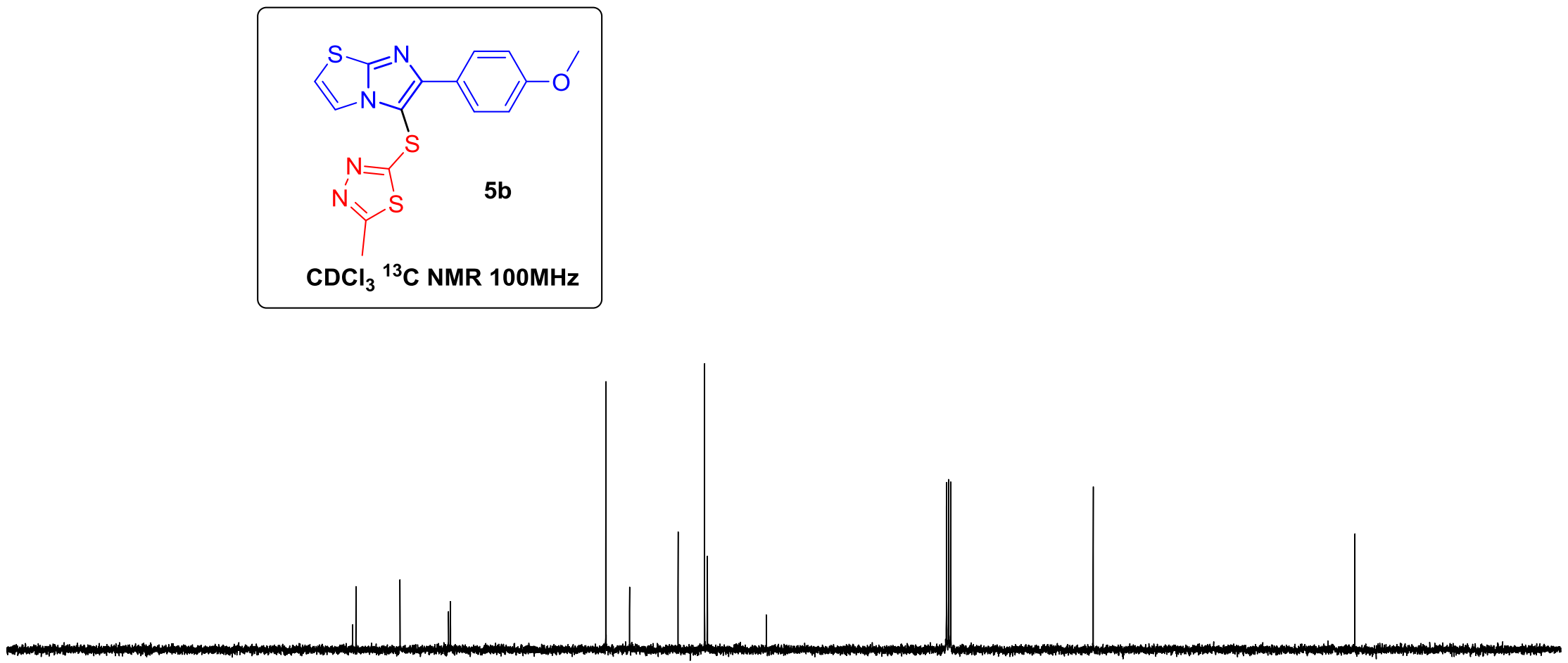

200

180

160

140

120

100

80

60

20 ppm 


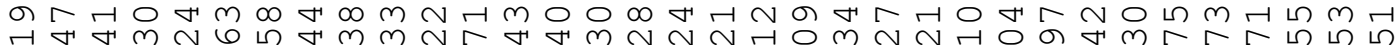
न o

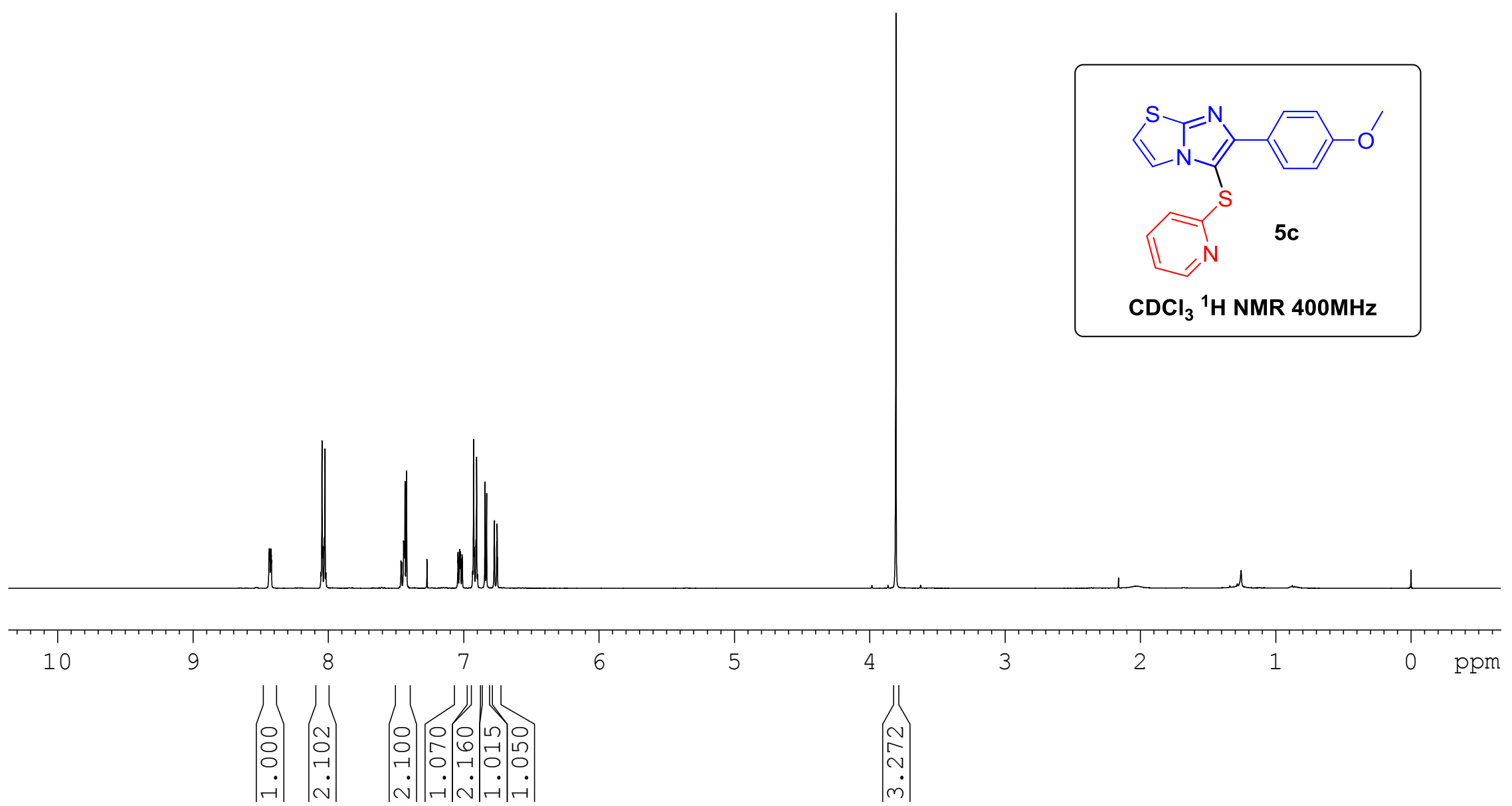




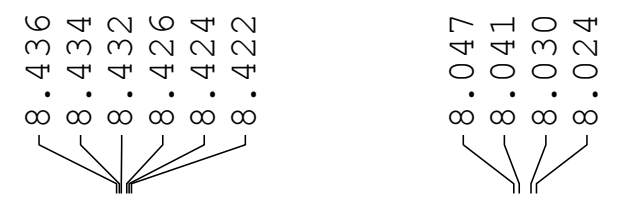

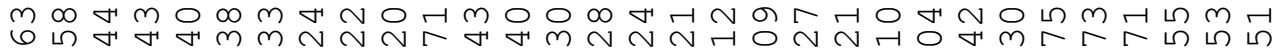
(

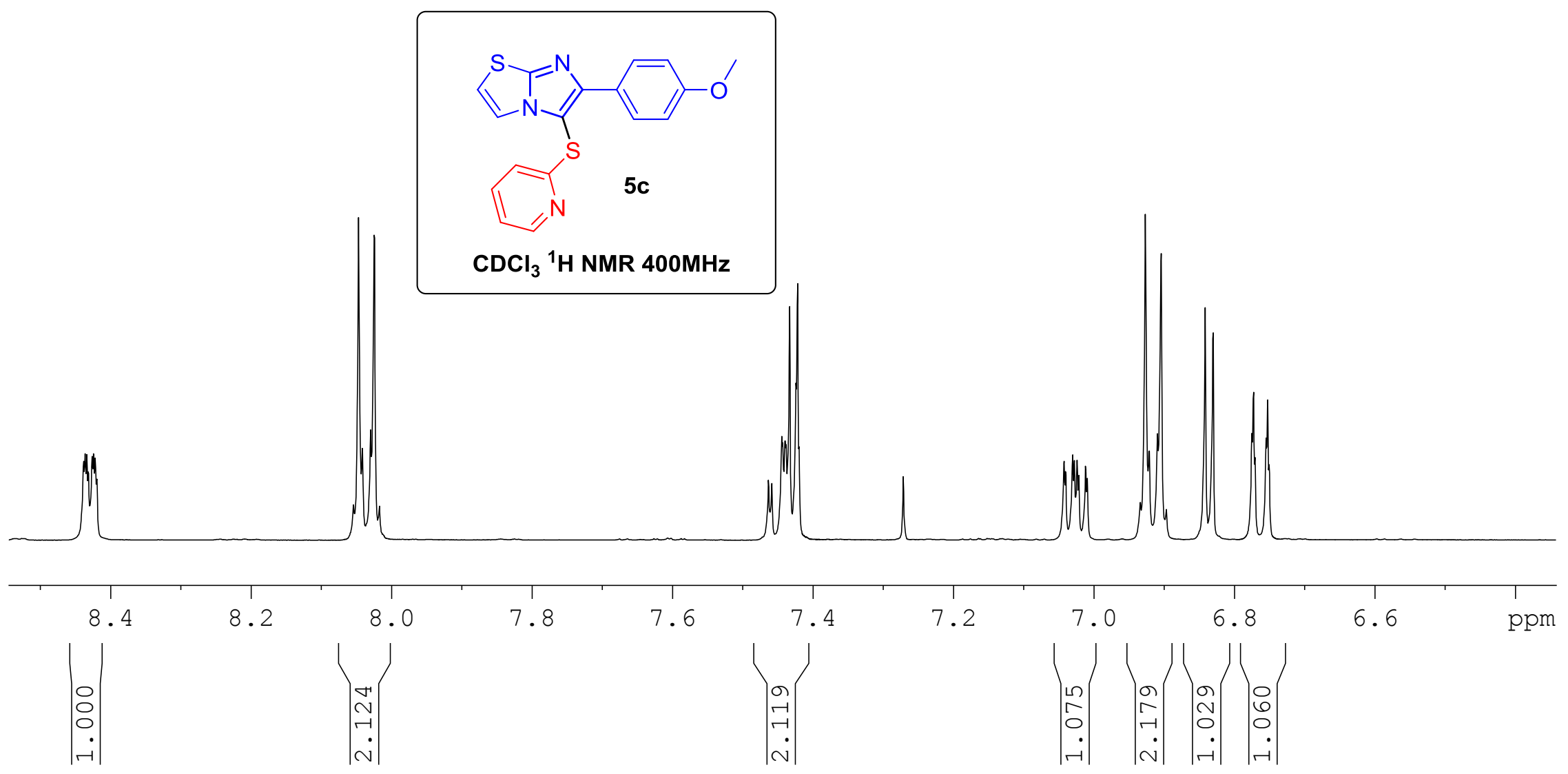




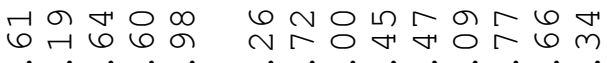

$\dot{0} \dot{0} \dot{0} \dot{0} \dot{0} \dot{0} \dot{\sim}$

صำ

V1/ 11111

\section{m๐ㅇ \\ 됴 \\ $Y$ \\ م.}

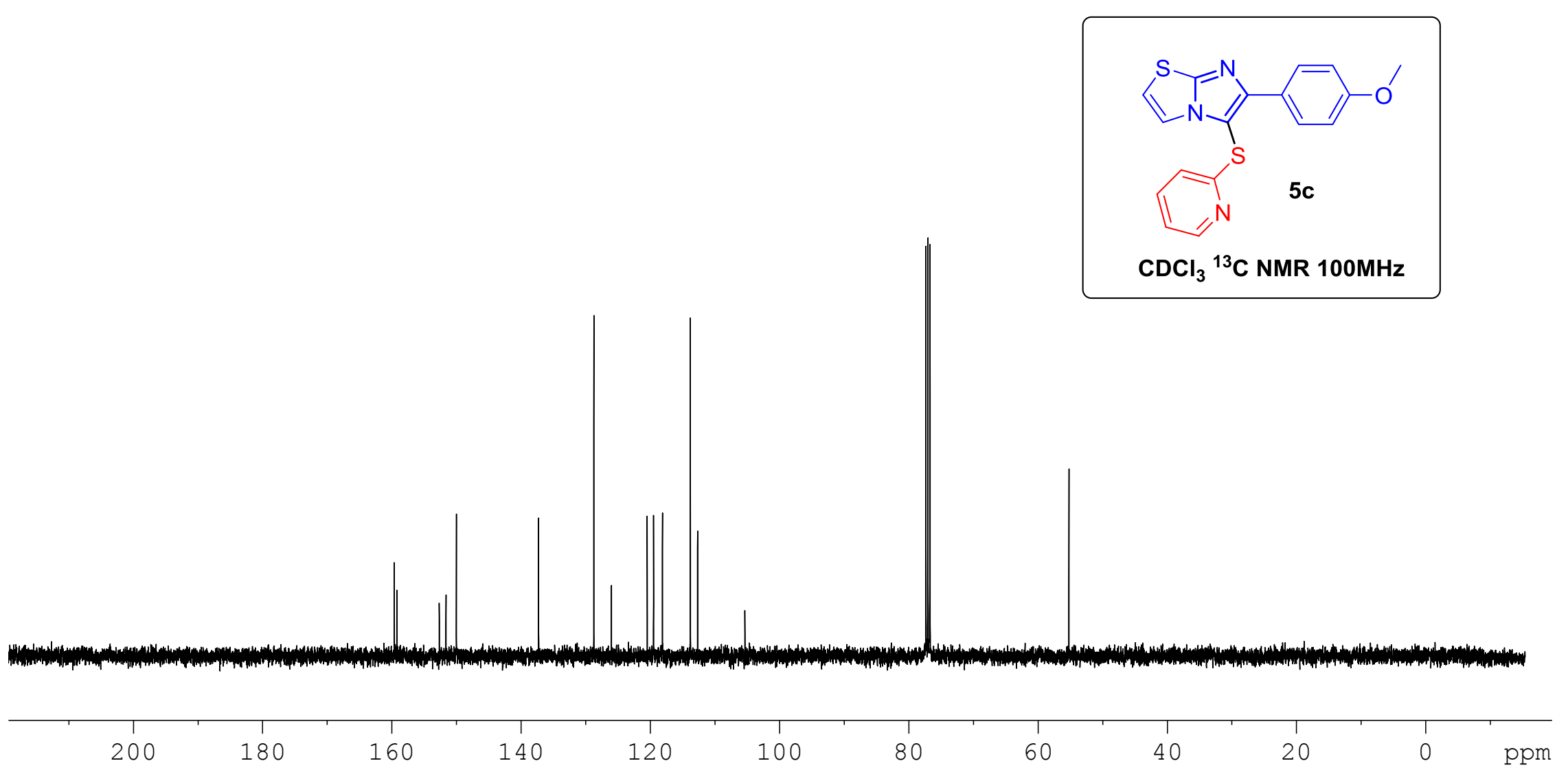




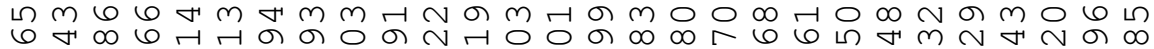

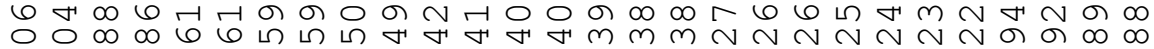

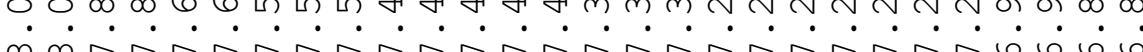

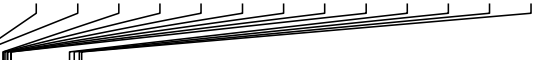

\section{1"}
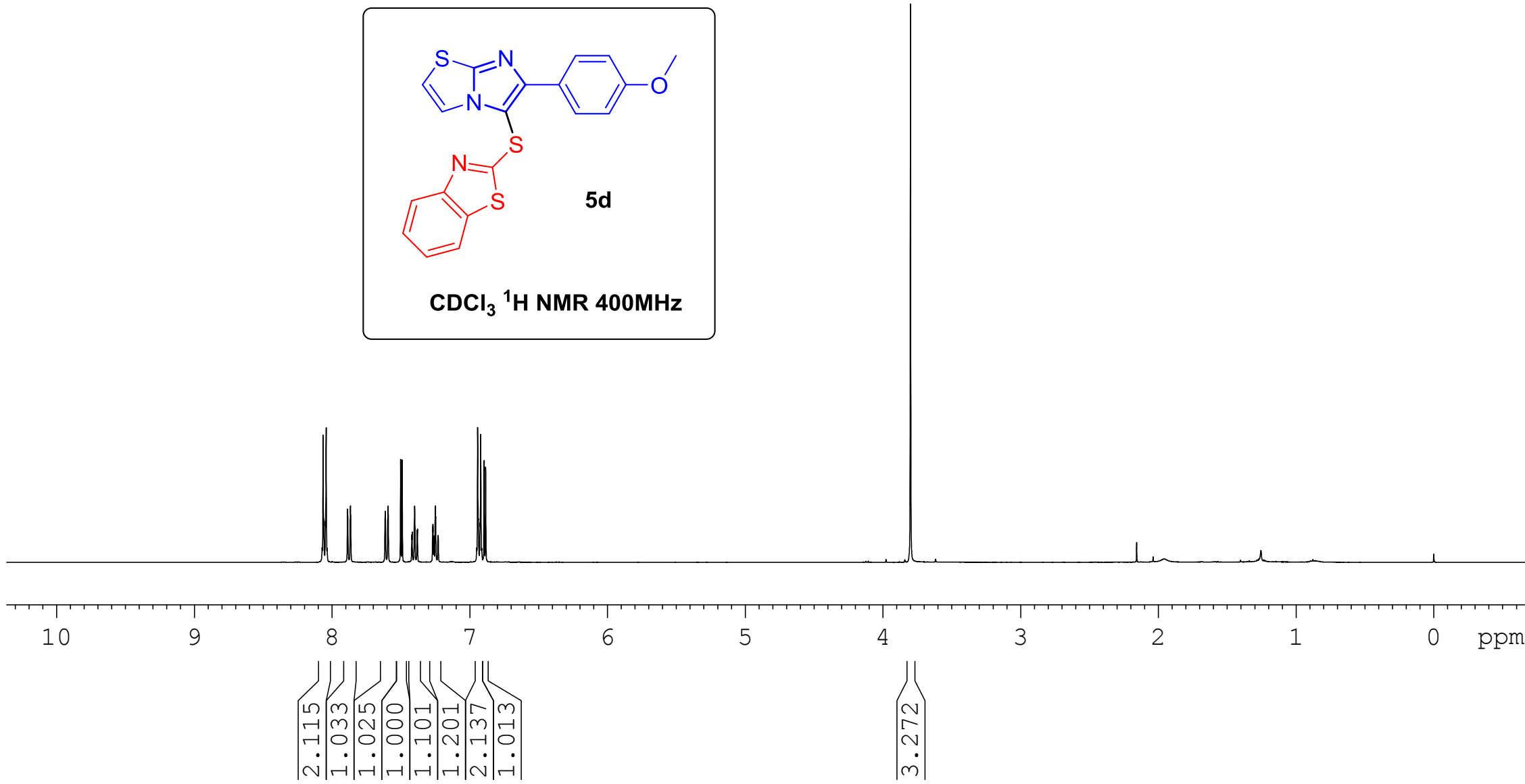


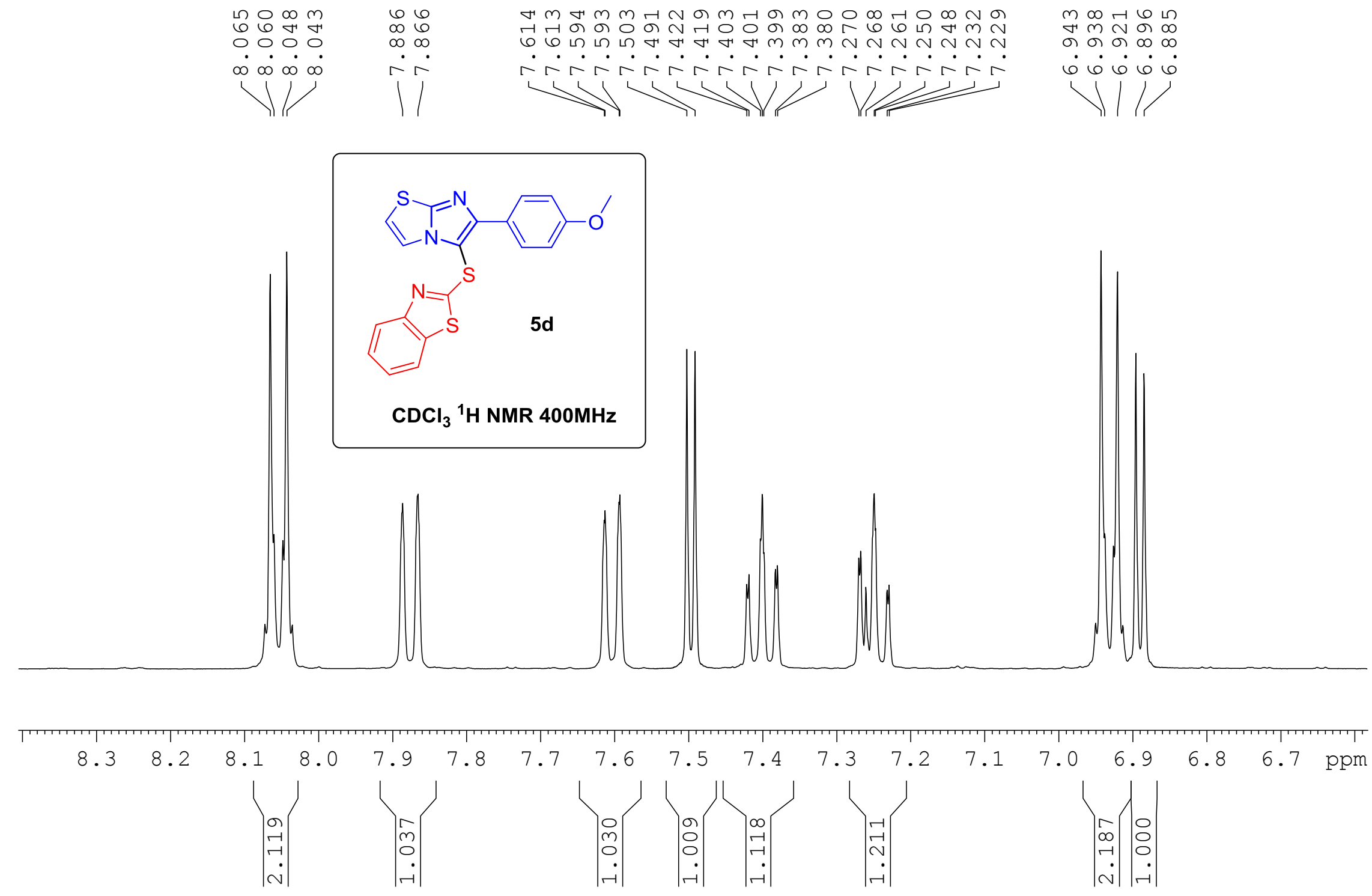


암 の

$\infty$.

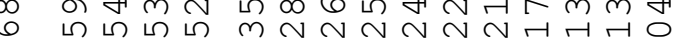

की

$1 / 11$

$\stackrel{n}{0} \circ \stackrel{\infty}{0}$

$\therefore \dot{0}$

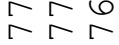

\/
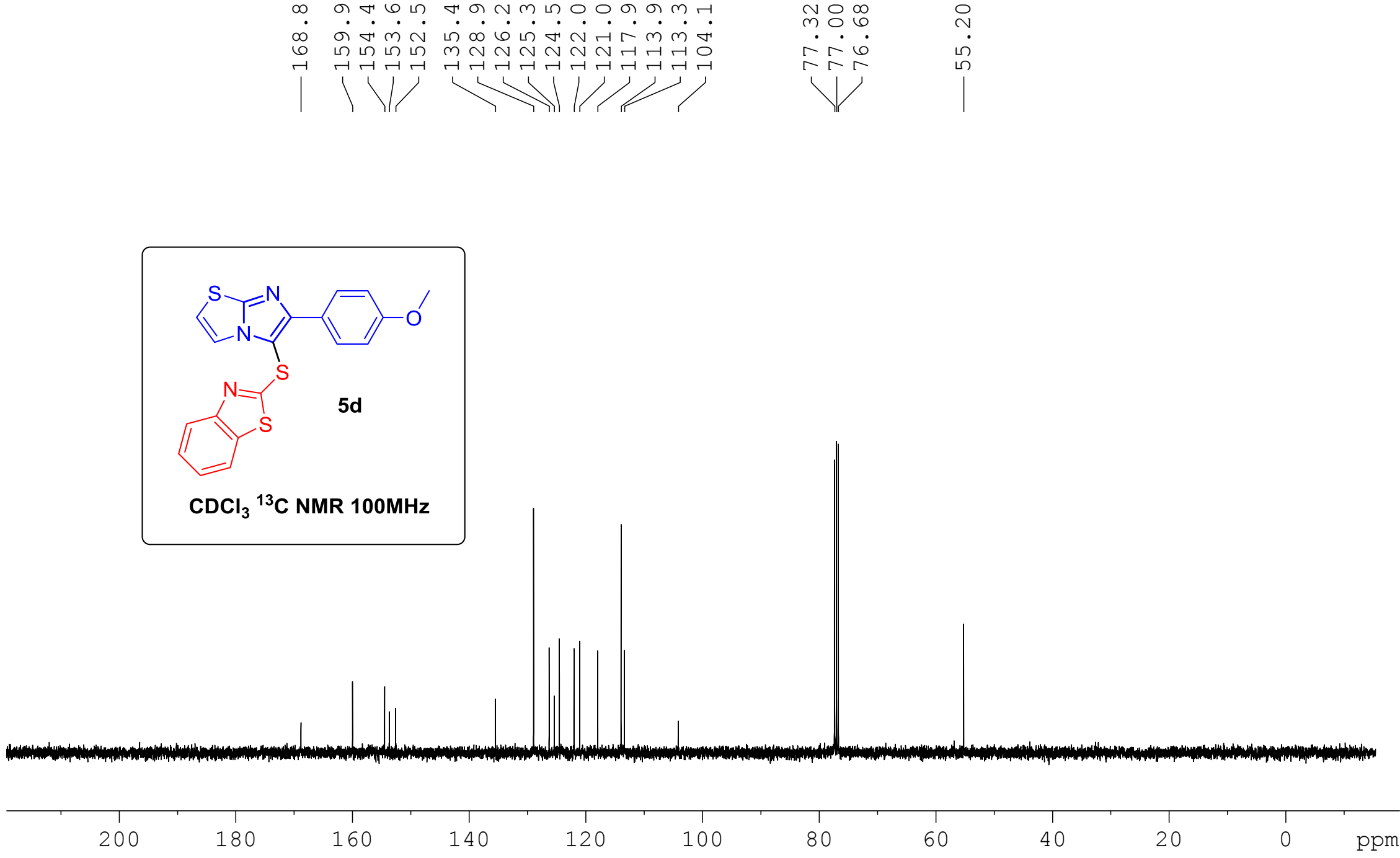


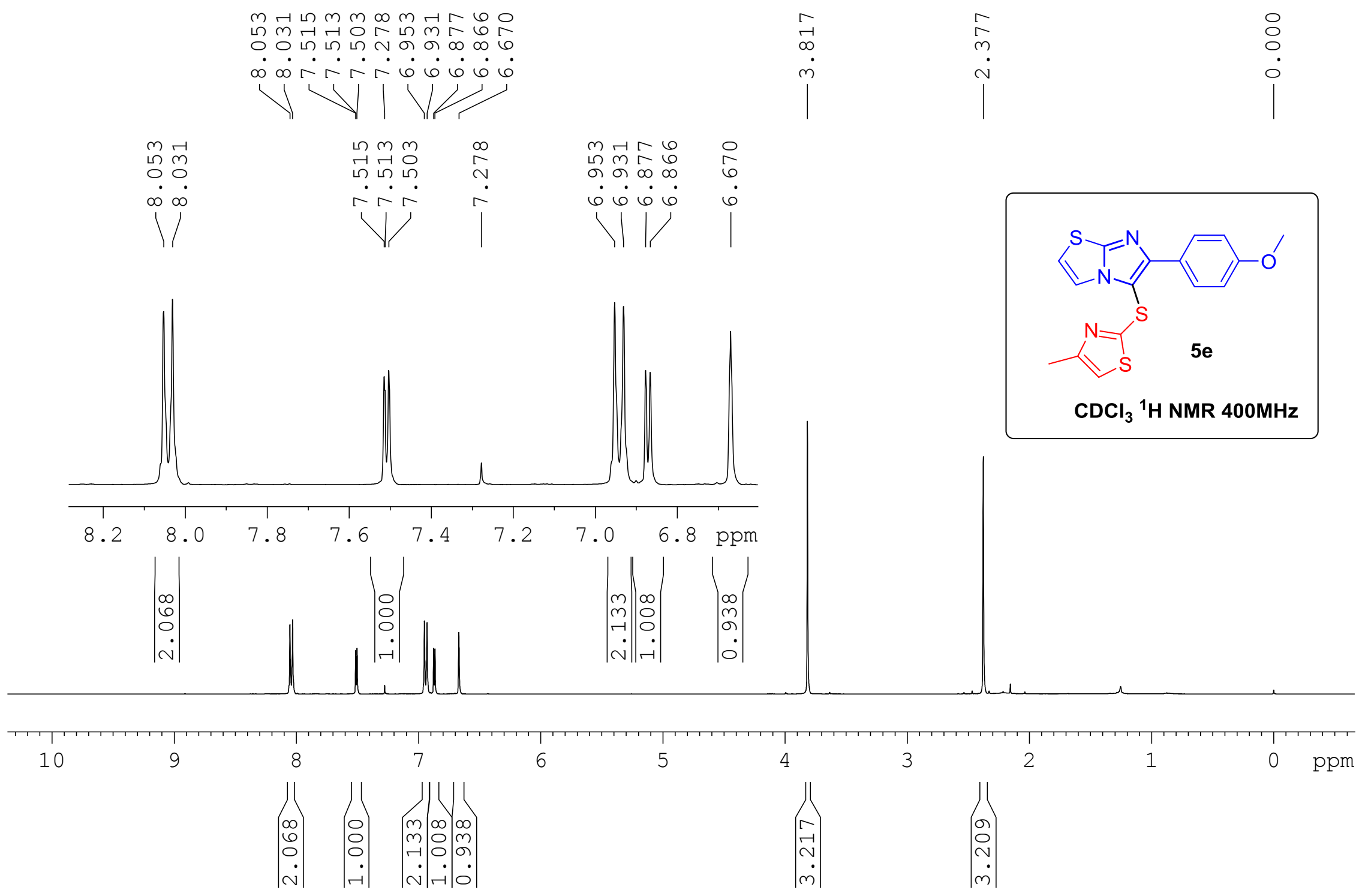



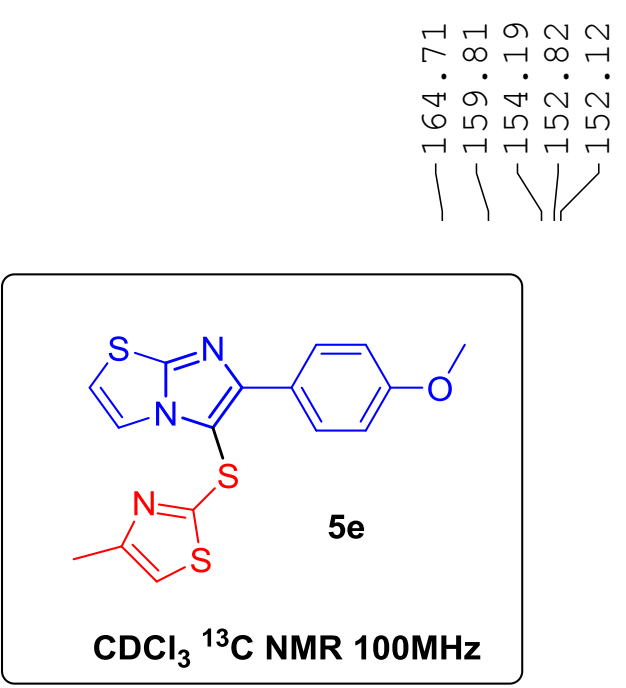

小

$\dot{\infty} \dot{\infty} \dot{\phi} \dot{m} \dot{m}$

$\sim \curvearrowright$

11

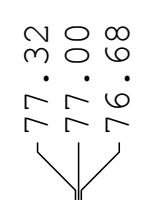

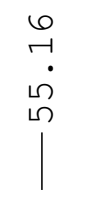

0
$\stackrel{1}{~}$
$\overrightarrow{1}$
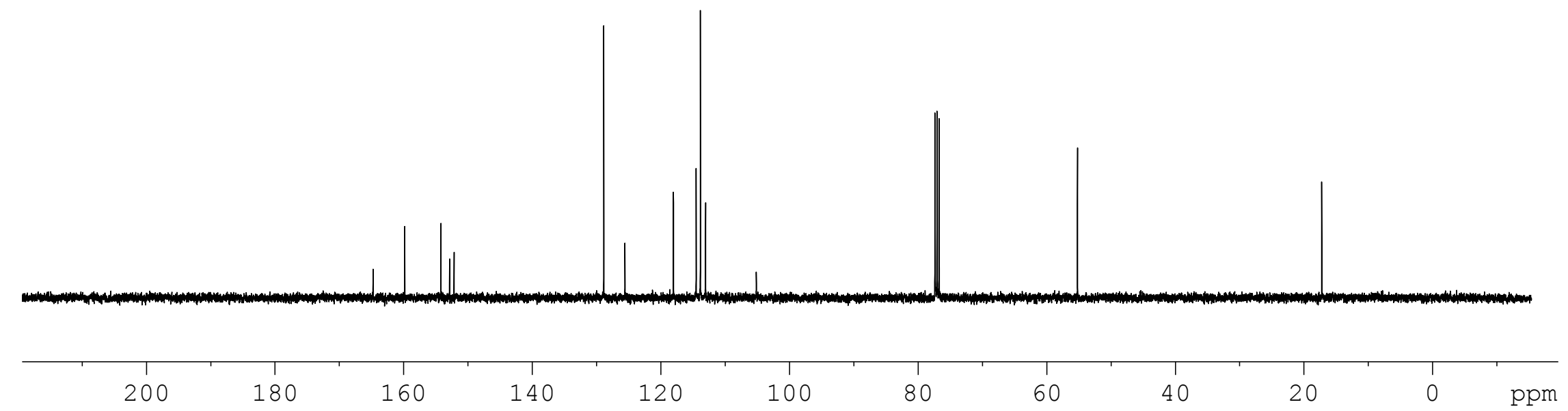


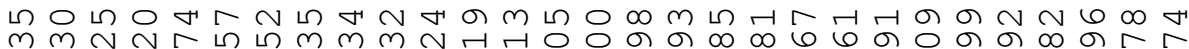

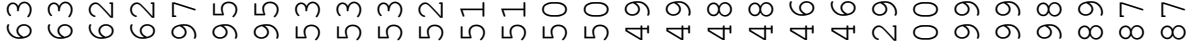

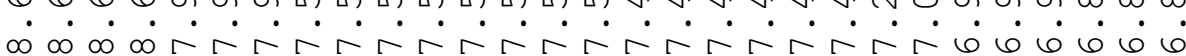
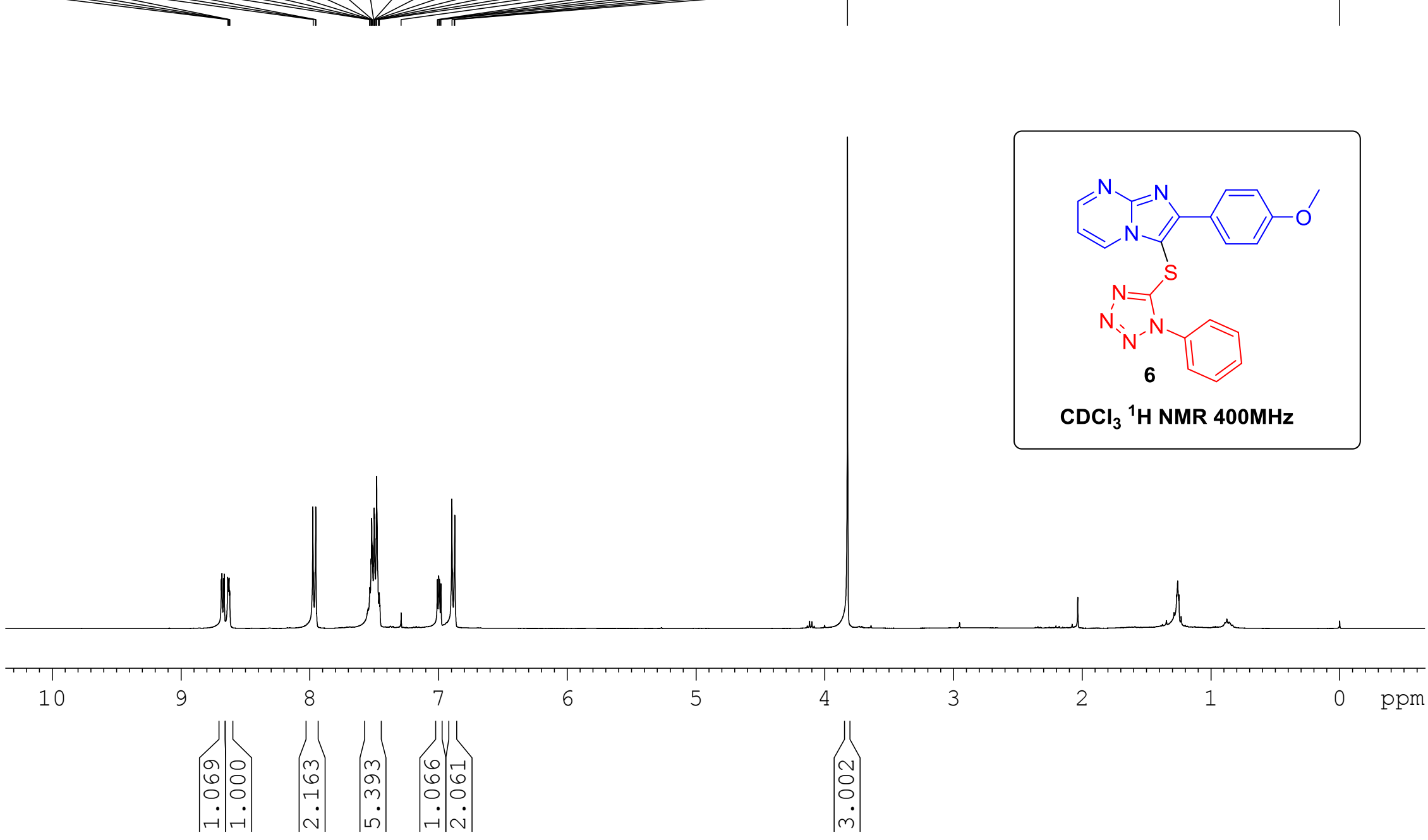


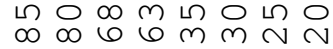

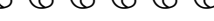
$\dot{\infty} \dot{\infty} \dot{\infty} \infty \dot{\infty} \dot{\infty} \dot{\infty} \infty \dot{0}$ $4>1$

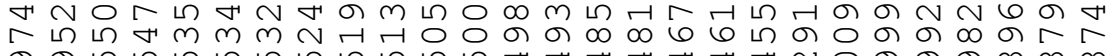
G.

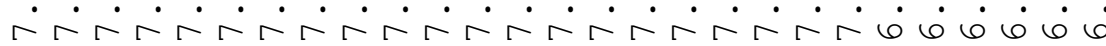

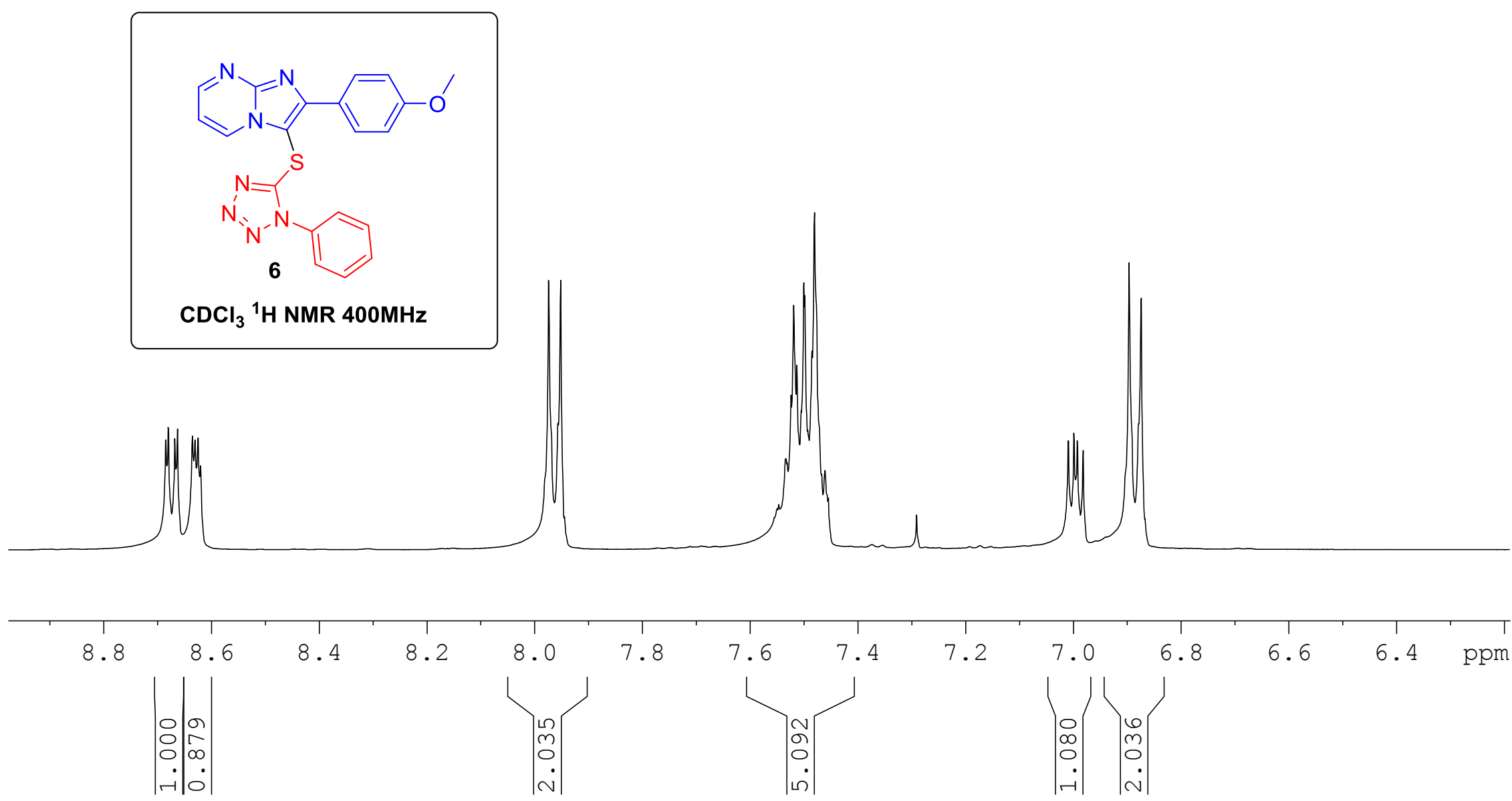


小 $\cdot \dot{0} \cdot \cdot \cdot \cdot \cdot \cdot \cdot \cdot \cdot \cdot \cdot \cdot \cdot \cdot$

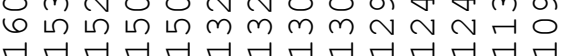
Mll 11

$\stackrel{\circ}{\sim}$
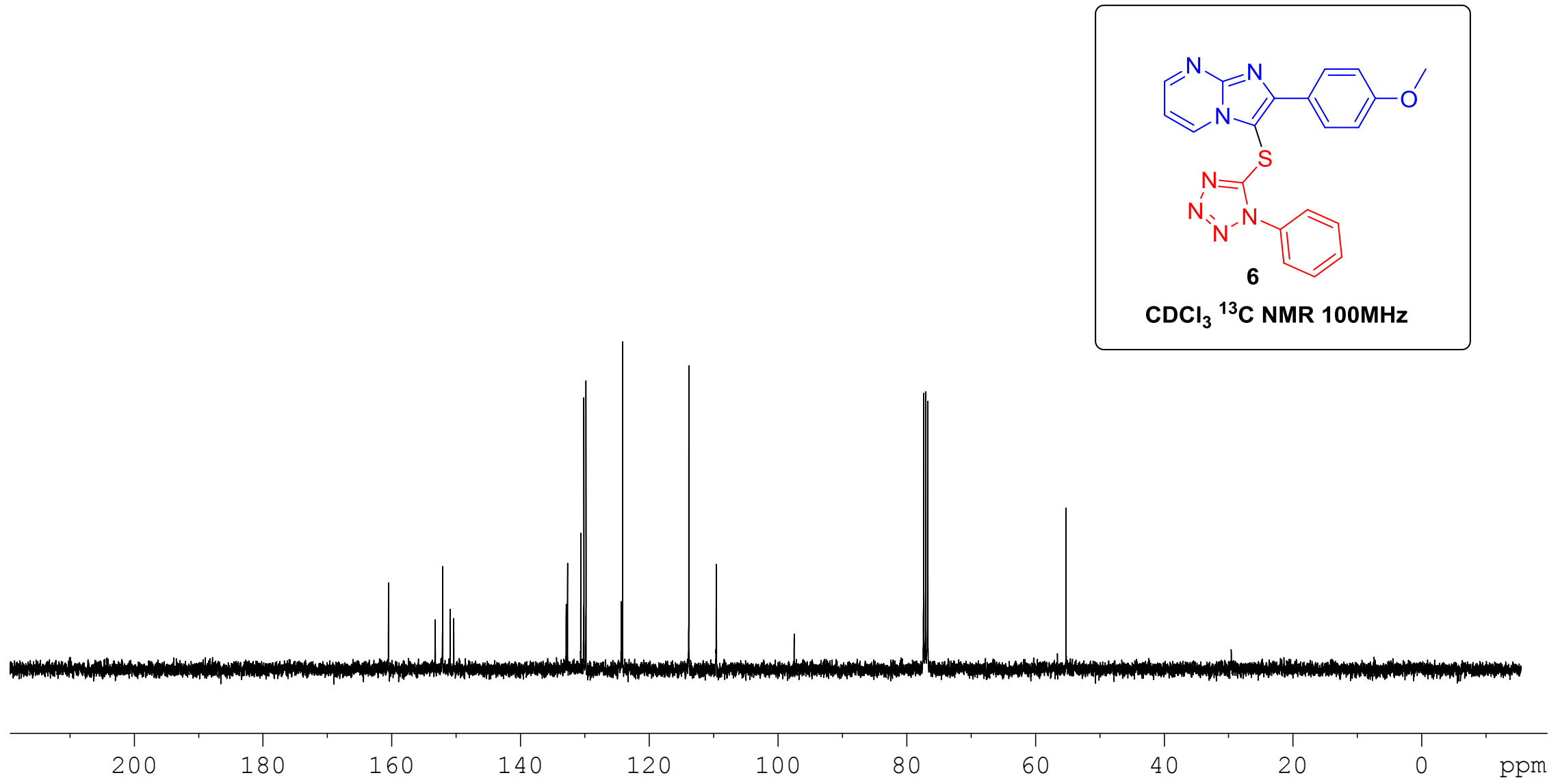


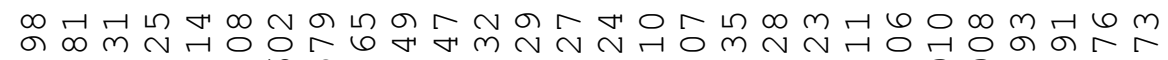

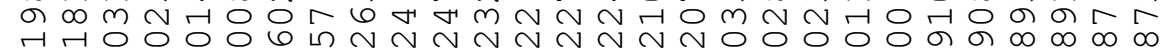

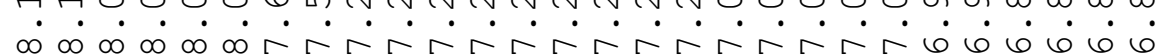

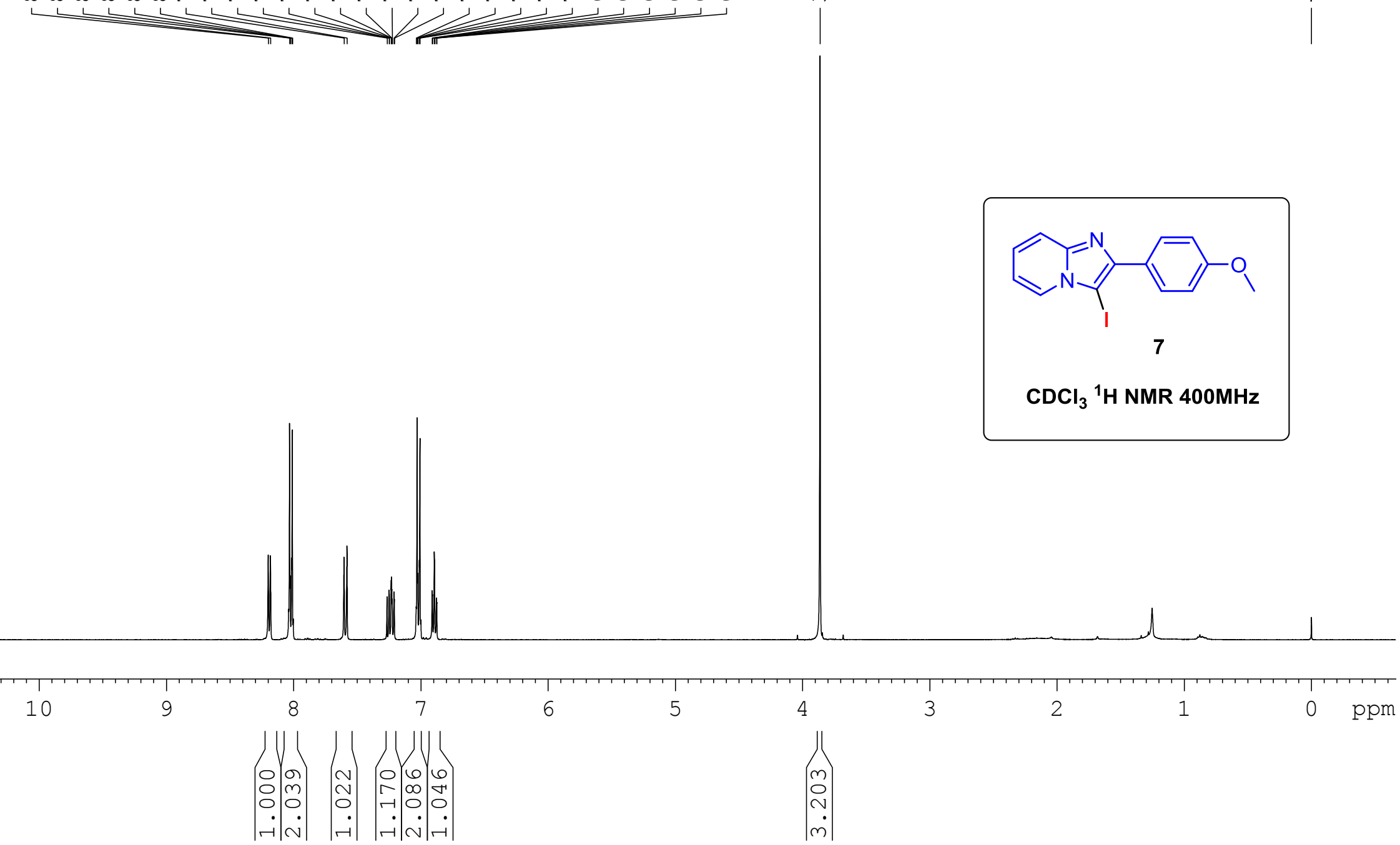




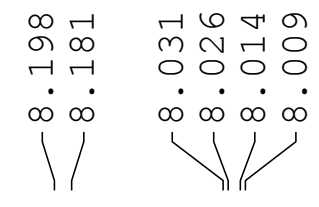

용ำ

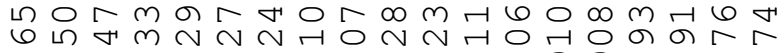
a

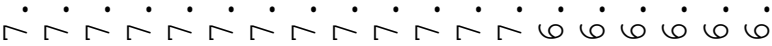

亦)

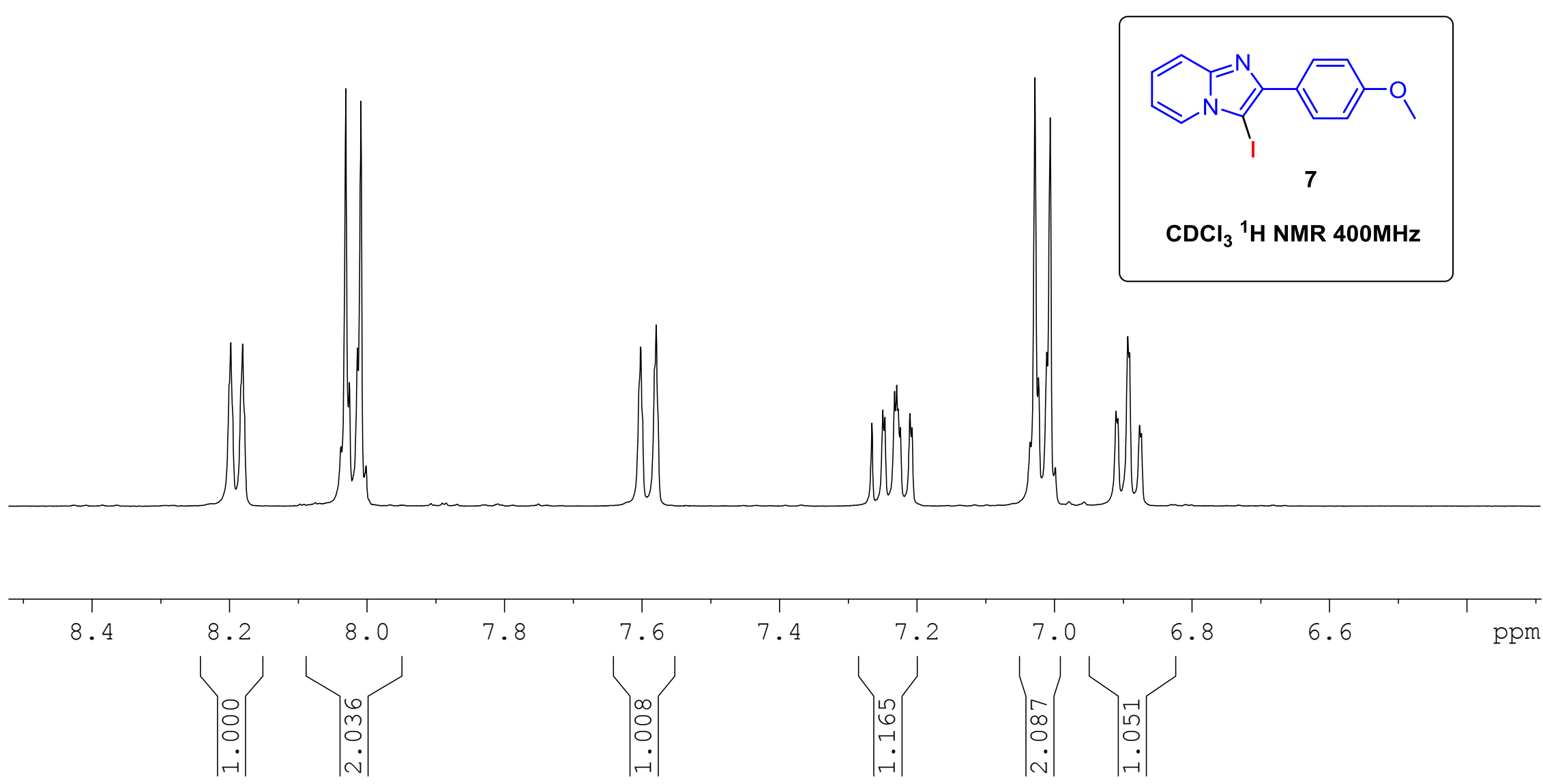


$\sim$
$\mathrm{m} \circ$

숭응

$\stackrel{10}{6}$

我 เ

11

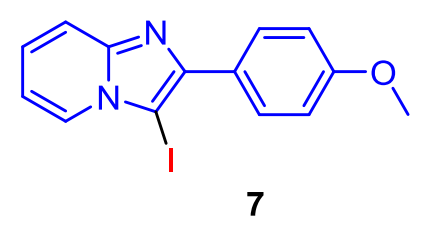

$\mathrm{CDCl}_{3}{ }^{13} \mathrm{C}$ NMR $100 \mathrm{MHz}$

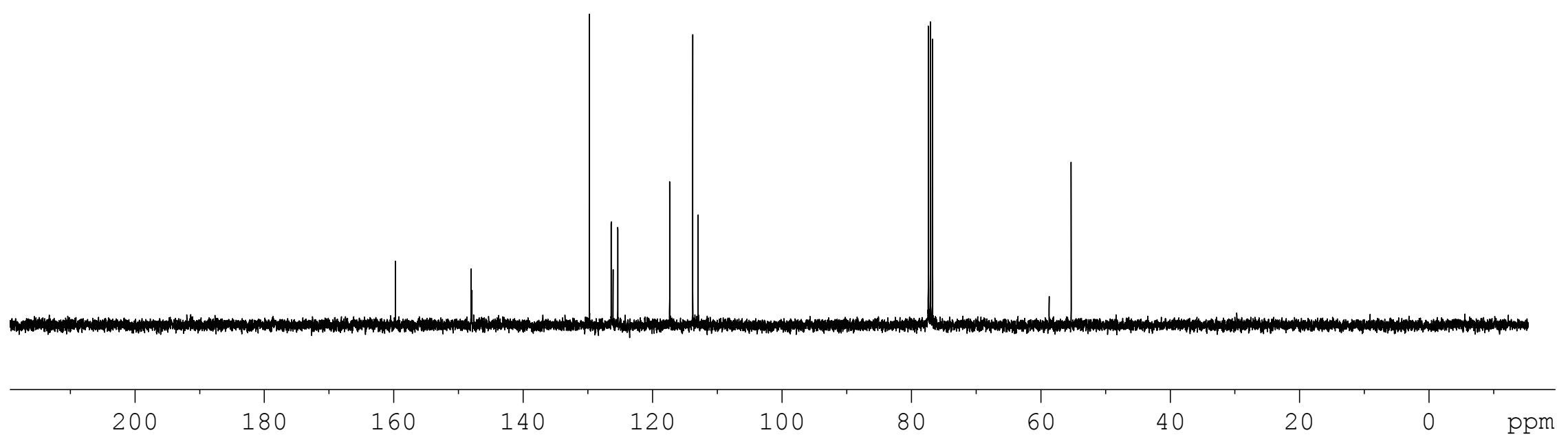

